NASA CONTRACTOR REPORT

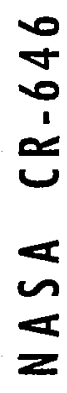

AN ANALYSIS OF CONFINED MAGNETOHYDRODYNAMIC VORTEX FLOWS

by David E. Loper

Prepared by

CASE INSTITUTE OF TECHNOLOGY

Cleveland, Ohio

for

NATIONAL AERONAUTICS AND SPACE ADMINISTRATION - WASHINGTON, D. C. • NOVEMBER 1966 


\title{
AN ANALYSIS OF CONFINED MAGNETOHYDRODYNAMIC VORTEX FLOWS
}

\author{
By David E. Loper
}

Distribution of this report is provided in the interest of information exchange. Responsibility for the contents resides in the author or organization that prepared it.

Prepared under Contract No. NsG 198/36-003-003 by CASE INSTITUTE OF TECHNOLOGY

Cleveland, Ohio

for

NATIONAL AERONAUTICS AND SPACE ADMINISTRATION

For sale by the Clearinghouse for Federal Scientific and Technical Informat on Springfield, Virginia 22151 - Price $\$ 3.25$ 
ABSTRACT*

The vortex flow of a viscous, incompressible and electrically conducting fluid confined between two finite flat plates (with a net radial mass flow) in the presence of an applied axial magnetic field 18 considered: The detalls of the velocity fleld including boundary layer blockage and redistribution of radial mass flow are of primary concern. The analysis is limited to the case of the separation distance between the two end plates being small compared with the radius of the plates; this allows the momentum equations to be uncoupled from the electromagnetic equations. The momentum equations are linearized and solved for the velocities and the pressure as Fourier series. The temperature and electromagnetic variables are then found. The velocity and temperature results are plotted and discussed, showing the influence of the magnetic effects upon the flow pattern.

*This investigation was submitted in partial fulfiliment of the requirements for the Degree of Doctor of Philosophy. 
ACKNOWLEDGEMENTS

The author wishes to thank Dr. Simon Ostrach, Professor of Engineering, who directed and supervised the present work, for his valuable suggestions which made the finished product possible. Acknowledgement is also due to the National Aeronautics and Space Administration for financing the present research project. 

TABLE OF CONTENTS

Page

ABSTRACT.

ACKNOWLEDGEMENIS.

iii

TABLE OF CONTENIS.

$\mathrm{v}$

LIST OF TABLES............................... vii

LIST OF FIGURES............................... vii

LIST OF SYMBOLS.............................. ix

Chapter

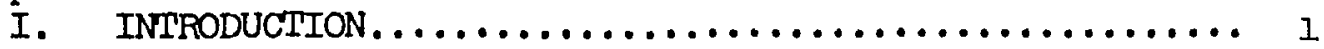

1. Some Previous Investigations............... I

2. Subject and Scope of Present Work............ 5

3. Assumptions and Lim1tations................ 7

4. A Discussion of the Boundary Conditions........ 8

A. The Velocity Boundary Conditions.......... 8

B. The Electromagnet1c Boundary Conditions.... 10

C. The Temperature Boundary Conditions....... 12

II. DEVELOPMENT OF EQUATIONS.................... 13

1. Basic Equations....................... 13

2. The Non-Dimensionalization................. 14

3. Expansion in Powers of $1 / s \ldots \ldots \ldots \ldots \ldots \ldots \ldots .20$

4. A Discussion of Types of Flow................ 36

III. EXACT SOLUTIONS OF THE LINEARIZED EQUATIONS......... 42

1. The Linearization....................... 42

2. Tangential Velocity Solution................. 46

3. Radial Velocity Solution.................... 51

4. A Further Simplification................. 56 
IV. CALCULATION OF THERMAL AND ELECTROMAGNETIC VARTABLES 64

1. Solution of Energy Equation for Prescribed End Wall Temperature.......................64

2. Solution of the Energy Equation for Adiabatic End Walls....................... 68

3. Calculation of Electromagnetic Variables.......69 69

A. Electric Currents....................696 69

B. Electric Fields......................70

C. Magnetic Fields.................... 72

V. DISCUSSION OF RESULTS...................... 74

1. Tangential Velocity...................... 75

2. Radial Velocity........................ 82

3. Temperature........................... 87

4. Concluding Remarks.................... 91

LIST OF REFERENCES. ........................... 94

APPENDIX I - Convergence Proof $. \ldots \ldots \ldots \ldots \ldots \ldots \ldots \ldots \ldots . . \ldots 6$

APPENDIX II - Analysis of Error..................... 100

APPENDIX III - Special Case Solutions................ 104

APPENDIX IV - Calculation of Power and Efficiency......... 119

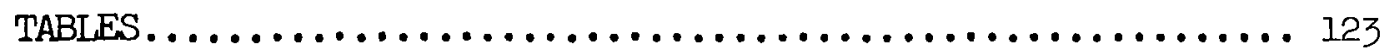

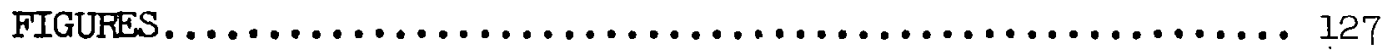




\section{LIST OF TABLES}

Table

I. Boundary Layer Thickness for Various Values of the Governing Parameters......................... 123

2. Comparison of Boundary Layer Thickness with Momentum Integral Analysis ................... 124

3. Boundary Layer Mass Flow and External Radial Velocity for Various Values of the Governing Parameters....... 125

4. Heat Transfer at the End Walls for Various Values of the Governing Parameters................ 126 
1. The Physical Configuration................... 127

2. Tangential Velocity versus $z$ for non Magnetic Case... 128

3. Tangential Velocity versus z Showing Magnetic Effects 129

4. Radial Velocity versus $z$ for Non-Magnetic Case...... 130

5. Radial Velocity versus $z$ Showing Effects of Velocity Ratio $\lambda \ldots \ldots \ldots \ldots \ldots \ldots \ldots \ldots \ldots \ldots \ldots$

6. Radial Velocity versus z Showing Magnetic Effects.... 132

7. Temperature versus $\mathrm{z}$ for $\mathrm{T}_{\mathrm{W}} / \mathrm{T}_{\mathrm{R}}=1.0 \ldots \ldots \ldots \ldots \ldots$

8. Temperature versus $\mathrm{z}$ for $\mathrm{T}_{W} / \mathrm{T}_{\mathrm{R}}=.2 \ldots \ldots \ldots \ldots \ldots \ldots$

9. The Function $f(x)$ versus $\dot{x}$ from Appendix II........ 135

10. The Error in Approximating $\mathrm{f}(\mathrm{x}) \ldots \ldots \ldots \ldots \ldots \ldots$ 


\section{LIST OF SYMBOLS}

Symbol

a a scalar

A a vector

$A_{r}, A_{\phi}, A_{z}$

$\mathrm{b}$

B

$\underline{B}$

B*

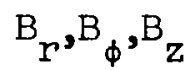

$\mathrm{B}_{r}^{*} \mathrm{~B}_{\phi}^{*} \mathrm{~B}_{\mathrm{Z}}^{*}$

$B_{i}$

$\mathrm{B}_{r 1}, \mathrm{~B}_{\phi 1}, \mathrm{~B}_{z 1}$

c

$c, c_{1}, c_{2}$

$\mathrm{C}_{\mathrm{n}}$

$d_{n}$

E

$\underline{E}$

$\underline{E}^{*}$

$E_{r}, E_{\phi}, E_{z}$

$E_{r}^{*}, E_{\phi}^{*}, E_{Z}^{*}$

$E_{i}$

$E_{r 1}, E_{\phi 1}, E_{z 1}$ components of a vector

separation distance between the two end plates

characteristic magnetic field strength

dimensionless magnetic field vector

dimensional magnetic fleld vector

dimensionless magnetic field components

dimensional magnetic field components

ordered magnetic field vector

ordered magnetic field component

specific heat.

constants

a constant

a constant

the Euler number $\left(=\mathrm{P} / \mathrm{oV}^{2}\right)$

dimensionless electric field vector

dimensional electric field vector

dimensionless electric field component

dimensional electric field component

ordered electric field vector

ordered electric field component 
LIST OF SYMBOLS (Contd)

Ec

The Eckert number $\left(=\mathrm{V}^{2} / \mathrm{cT}_{\mathrm{R}}\right)$

$\stackrel{2}{\Delta \mathrm{E}}$ dimensionless external potential difference $\Delta \tilde{E} *$ dimensional external potential difference $f(x)$ a function defined by equation III-45 $f_{1}, f_{2}, f_{3}, f_{4}, f_{5}$ functions of the radius a function of the radius $g_{1}, g_{2}$ functions of the radius

$G_{1}$ $\pi^{2} s^{2} / R e+R m N$ $G_{n}$ $\pi^{2} s^{2} / \operatorname{Re}(2 n-1)^{2}+\operatorname{RmN}$

$\mathrm{H}$ dimensionless total power dimensional total power

$\tilde{\mathrm{H}}$ dimensionless useful power dimensional useful power

$\mathrm{Ha}$ the modified Hartmann number $(=s / \sqrt{\operatorname{ReRmN}})$ $I_{0}, I_{1}$ modifled Bessel functions of the first kind dimensionless external electric current dimensional external electric current dimensionless electric current vector dimensional electric current vector $\mathrm{J}_{r}, \mathrm{~J}_{\phi}, \mathrm{J}_{z}$ dimensionless electric current component $\mathrm{J}_{\mathrm{r}}^{*}, \mathrm{~J}_{\phi}^{*}, \mathrm{~J}_{\mathrm{Z}}^{*}$ dimensional electric current component Ji ordered electric current vector $J_{\text {ri }}, J_{\phi 1}, J_{z I}$ ordered electric current component $k$ thermal conductivity 


\section{LIST OF SYMBOLS (Contd)}

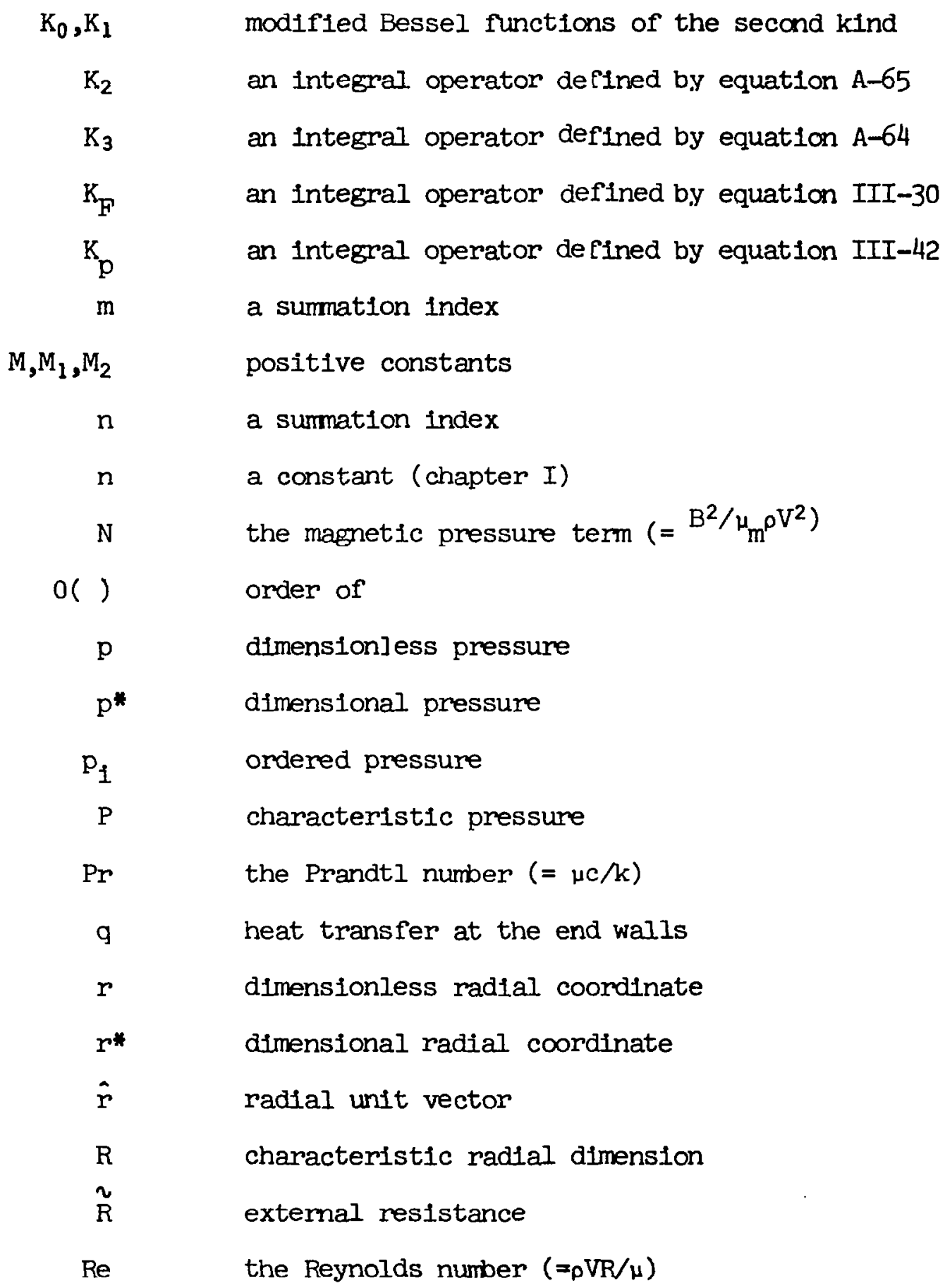


LIST OF SYMBOLS (Contd)

Rn

$\mathbf{s}$

$t$

$\mathrm{T}$

T*

$T_{1}$

$\mathrm{T}_{\mathrm{R}}$

$T_{W}$

u

*

$u_{1}$

$\bar{u}_{n}$

v

V*

$\mathrm{v}$

$v^{*}$

$v_{1}$

$\overline{\mathrm{v}}_{\mathrm{n}}$

V

W

W*

$w_{1}$

$\mathrm{x}$ the magnetic Reynolds number $\left(=\mu_{m} \sigma R V\right)$

the shape parameter $(=\mathrm{R} / \mathrm{b})$

time

dimensionless temperature

dimensional temperature

ordered temperature

inlet temperature

end wall temperature

dimensionless radial velocity

dimensional radial velocity

ordered radial velocity

transformed radial velocity

dimensionless velocity vector

dimensional velocity vector

dimensionless tangential velocity

dimensional tangential velocity

ordered tangential velocity

transformed tangential velocity

characteristic velocity

dimensionless axial velocity

dimensional axial velocity

ordered axial velocity

a variable 
LIST OF SYMBOLS (Contd)

y

$\mathbf{z}$

$\mathbf{z}^{*}$

$\hat{\mathbf{z}}$

a

$\alpha_{n}$

B

$\gamma_{n}$

$\varepsilon$

$n$

$\theta$

$\Theta$

$\lambda$

$\mu$

$\mu_{\mathrm{m}}$

$v_{n}$

$\pi$

$\rho$

$\rho_{e}$

$\sigma$

$\hat{\phi}$

$\Phi *$ a variable

dimensionless axial coordinate

dimensional axtal coordinate

axtal unit vector

external current parameter (defined by equation II-73)

a constant

efficiency

a constant

the ratio of the radius of the inner cylinder to the radius of the outer cylinder

a dumny integration variable

$\pi^{2} s^{2} / \operatorname{Re}$

a function defined by equation A-56

the ratio of the inlet tangential velocity to the inlet radial velocity

viscosity

magnetic permeability

a constant

3.14159...

density

electric charge distribution

electric conductivity

tangential unit vector

the dissipation function 


\section{LIST OF SYMBOLS (Contd)}
$x$
a function
$\Psi$
a function defined by equation $\mathrm{A}-67$
$\omega_{n}$
a constant

SUPERSCRIPTS

*

'

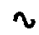

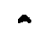

SUBSCRIPIS

$\begin{aligned} r, \phi, z & \begin{array}{l}\text { coordinate directions } \\ n\end{array} \\ - & \text { vector quantity } \\ 0,1,2, \ldots & \text { ordered component } \\ R & \text { at radius } r^{*}=R \\ w & \text { at end wall }\end{aligned}$

OPERATORS

curl A

$\hat{-\hat{r}} \frac{\partial A_{\phi}}{\partial z}-\hat{\phi}\left[\frac{1}{r} \frac{\partial}{\partial r}\left(A_{z} r\right)-\frac{\partial A_{r}}{\partial z}\right]+\hat{z} \frac{\partial\left(A_{\phi} r\right)}{r \partial r}$

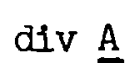

dimensional variable

differentiation

pertaining to external electric circuit

unit vector 
LIST OF SYMBOLS (Contd)

$\frac{D}{D t} \underline{A} \quad\left[\frac{\partial}{\partial t}+u \frac{\partial}{\partial r}+w \frac{\partial}{\partial z}\right][\underline{A}]$

$\operatorname{grad} a \quad\left[\hat{r} \frac{\partial}{\partial r}+\hat{z} \frac{\partial}{\partial z}\right] a$

$$
\begin{aligned}
\nabla^{2} \underline{A} & \hat{r}\left[\frac{\partial^{2} A_{r}}{\partial r^{2}}+\frac{\partial}{\partial r}\left(\frac{A r}{r}\right)+\frac{\partial^{2} A_{r}}{\partial z^{2}}\right]+\hat{\phi}\left[\frac{\partial^{2} A_{\phi}}{\partial r^{2}}+\frac{\partial}{\partial r}\left(\frac{A_{\phi}}{r}\right)+\frac{\partial^{2} A_{\phi}}{\partial z^{2}}\right] \\
& +\hat{z}\left[\frac{\partial^{2} A_{z}}{\partial r^{2}}+\frac{1}{r} \frac{\partial A_{z}}{\partial r}+\frac{\partial^{2} A_{z}}{\partial z^{2}}\right]
\end{aligned}
$$

XV 


\section{CHAPTER I.}

\section{INIRODUCTION}

\section{Some Previous Investigations}

Vortex flows have been of continuing interest to flutd dynamicists for some time. Recently, interest has been focused upon the problems of vortices confined within finite bounding walls and of vortex flows in the presence of magnetic flelds. This interest has been stimulated in part by the possibility of power generation by means of a magnetohydrodynamic vortex power generator [1] and by the possibility of developing a gaseous fission rocket [2].

To gain an understanding of the nature of the flow of a vortex in the presence of bounding walls, consider a free vortex (tangential velocity $1 / r$ ) near a stationary flat plate which is perpendicular to the vortex axis. The radial pressure gradient, $d p / d r$, away from the plate varies as $v^{2} / r$ or as $1 / r^{3}$. Near the flat plate, the tangential velocity is slowed forming a boundary layer. The external radial pressure gradient is impressed upon this boundary layer. Within the boundary layer, the tangential velocity is insufficient to counterbalance this pressure gradient. This results in a pressure defect which drives the fluid within the boundary layer radially inward. In this way strong radial mass 
flow is generated near the plate. This radial flow must be compensated by a mass flow Into the boundary layer from the outer flow. For vortex flow near a single flat plate, conservation of mass is sat1sfled by an axial flow from infinity; the radial velocity outs1de the boundary layer is assumed to be zero. In an attempt to describe accurately the nature of this boundary layer upon the single flat plate in the presence of a vortex, a considerable number of momentum integral analyses have been performed $[3,4,5]$. Lewellen and King [6] have analyzed this single plate problen with the addition of an applied axial magnetic fleld. They performed a momentum integral analysis of a vortex flow (tangential velocity $\sim r^{n}$ ) over a finite flat plate under the restriction that the radial electric current outside the boundary layer be a zero.

In a problem with vortex motion occurring in the confined region between two stationary flat plates (see flgure 1), the flow picture must be different. The slowing of the tangential velocity forming boundary layers and the accumulation of radial mass flow withtn these boundary layers due to the pressure defect occurs as described above. If the action of viscosity is so great as to cause the boundary layers to occupy an appreclable fraction of the volume of the confined region, the vortex motion may be greatly affected. This effect is referred to as boundary layer blockage. In the single plate case, the boundary layer mass flow is campensated by an axial flow toward the plate. W1th the add- 
tion of a second plate, this axial flow is blocked; conservation of radial mass flow within the boundary layers must be satisfled by a decrease in the radial velocity in the inviscid region between the two plates. Therefore, the radial velocity outside the boundary layers cannot be assumed to be zero as was done in the single plate problem described above or else it will be impossible to satisfy conservation of mass.

In order to maintain physically the vortex flow, the net radial velocity must be directed toward the axis of rotation. A large fraction of the total radial mass flow may be drawn into the boundary layers by the action of the pressure defect. If the fraction of radial mass flow diverted into the boundary layers is greater than unity, the radial velocity outside the boundary layers must change direction from toward the vortex axis to away from the axis. In this case, the vortex motion breaks down and the flow picture becomes more complicated.

Many of the single plate analyses referenced above have been made in an attempt to describe the flow of a vortex confined by two flat plates. However, since the conditions of the two problems are basically different as described above, it is not apparent that an analysis of the single plate problem yields results applicable to the two plate problem.

Vogelpchl [7] was the first investigator to attempt an analysis of a confined vortex. He considered the flow of a vis- 
cous incompressible vortex confined by two flat plates placed perpendicularly to the vortex axis (figure 1). He obtained an exact solution for the tangential velocity under the restrictive assumptions that the radial velocity was completely independent of the axial coordinate (that is, it varied inversely as the radius) and that the axial velocity was zero. The assumption that the radial velocity does not vary with the axial coordinate precludes the accumulation of the radial mass flow within the boundary layers due to the action of the pressure defect. Thus his solution is not an accurate representation of the vortex flow between two flat plates.

Loper [8] analyzed the two plate problem for the case of the plates relatively close together using a momentum integral analysis. The integral method was modified from the form used in the single plate analyses to account for the fact that the radial velocity outside the boundary layers is not zero. This method is a more accurate representation than Vogelpohl's in that it allows redistribution of radial mass flow into the boundary layers.

Rosenzweig, Lewellen and Ross [9] also analyzed the two plate configuration. They essentially limited their analysis to the case where the tangential velocity is much larger than the radial velocity and the separation distance between the plates is larger than the radius of the plates. Thetr expansion technique forced them to divide the region of interest into inviscid, boundary layer and central core regions and to patch solutions at 
the boundarles of these regions. Unfortunately, they were not able to obtain good experimental correlation.

The momentum integral technique, used almost exclusively for the above boundary layer analyses, does not yleld many details of the flow fleld. Also its accuracy depends upon the proper cholce of velocity profiles in the boundary layer. With the addition of magnetic effects to the two plate problem, this technique becomes too cumbersome to be employed successfully. Therefore, another method of solution will be employed in the analysis of the governing equations in Chapter III. This is the linearization method of Oseen and Targ [10]. This method does not require the boundary layer velocity profiles to be assumed beforehand and it yields more detailed information concerning the flow.

\section{Subject and Scope of Present Work}

In the following, the vortex f'low of a viscous, incompressible, and electrically conducting fluid between two finite flat plates ( $f$ igure 1 ) in the presence of an applied axial magnetic field will be analyzed. The region of interest is bounded radially by two coaxial cylinders which also act as electrodes connected by an external resistive load. The influence of the two end plates upon the vortex flow is of particular interest.

The basic governing equations, Navier-Stokes, conservation of mass, energy, Maxwell's and Ohm's Law, will be simplified with 
the help of certain assumptions enumerated in the following section. These equations will then be further simplified by considering only the case for which the radius of the outer cylinder (R) Is much larger than the separation distance between the two plates (b). The boundary layer interaction is most pronounced for this configuration. Also, this geometric restriction allows a considerable simplification in the governing equations; it is possible to express the magnetic body force terms in terms of the velocity components and thus eliminate Maxwell's equations and Ohm's Law from immediate consideration. In addition, this assumption allows a unified analysis of the region of interest rather than a split-up requiring patching of solutions.

The non-linear terms of the simplified Navier-Stokes equat1ons will be Iinearized in the manner of Oseen and Targ [10]. This Iinearization uncouples the equations to facilitate their solution. These linearlzed equations may be solved exactly to yield Fourler series solutions for the velocity profiles. These profiles will be calculated numerically and plotted on graphs for various values of the governing parameters.

With the velocity distribution known, the energy equation may be simpliffed and solved for the temperature distribution. Finally, parameters such as voltage, electric current and magnetic fields will be obtained. 


\section{Assumptions and Limitations}

A number of assumptions will be made concerning the nature of the working fluid. Some of them are well justified on physical grounds but others must be rather arbitrarily made in order that the problem become mathematically tractable.

It is assumed that:

a. The fluid is Newtonian; that is; the fluid is of one species which obeys the Navier-Stokes equations.

b. The flow is steady and axially symmetric.

c. The flow is laminar。

d. The fluid is incompressible.

e. The properties of the fluid are constant.

f. No body force other than the magnetic body force acts on the fluid.

g. The fluid is non-magnetic and has no polarization.

h. The conductivity is a scalar constant。

i. Radiation effects are negligible.

j. The flow speed is small compared to the speed of light.

The first assumption is basic to fluid flow problems but might not be valid if lonization and dissociation effects become important. The second is well founded"physically due to the nature of the boundary conditions prescribed below.

The validity of assumption c depends upon the value of the Reynolds number. If the flow is turbulent; the solution may be 
roughly approximated by a laminar solution by the use of a combined eddy viscosity. Assumption $d$ is very good if the working fluid is a conducting liquid but is not good if the fluid is a hot gas. Assumptions $d$ and $e$ allow the energy equation to be uncoupled and make the set of equations a bit more manageable.

Assumptions $f$ and $g$ form part of the magnetohydrodynamic approximation and are generally true for most fluids. Assumption $h$ means that the Hall and ion slip effects are neglected. The last assumption is not good for very high temperatures but must be made for mathematical simplicity.

4. A Discussion of the Boundary Conditions

A. The Velocity Boundary Conditions

The end plates are stationary and impermeable and there is no slip of the fluid at the plates. With the end plates separated by $a$ distance $b$ and with the coordinate system shown in figure 1 , these conditions may be expressed mathematically as:

$$
\text { At } \mathrm{z}^{*}=0 ; \quad \mathrm{u}^{*}=\mathrm{v}^{*}=\mathrm{w}^{*}=0
$$

and

$$
\text { At } z^{*}=\mathrm{b} ; \quad \mathrm{u}^{*}=\mathrm{v}^{*}=\mathrm{w}^{*}=0
$$

where the asterisks denote dimensional variables and all symbols are defined in the List of Symbols.

Since the configuration is symmetric about the midplane between the two end plates, further conditions may be expressed at $z^{*}=b / 2$ : 


$$
\text { At } \quad z^{*}=b / 2 ; \quad \frac{\partial u^{*}}{\partial z^{*}}=\frac{\partial v^{*}}{\partial z^{*}}=w^{*}=0
$$

These conditions are not independent of those stipulated above but are more convenient to apply in certain circunstances.

Let the region of interest be bounded radially by the two cylInders at $r^{*}=\varepsilon R$ and $r^{*}=R$ where $\varepsilon$ is a positive number less than one. In order physically to maintain the vortex flow there must be a radially inward velocity. However, if the fraction of radial velocity drawn into the boundary layers is greater than one, the radial velocity outside the boundary layer will reverse direction and the vortex will break down. This vortex breakdown occurs first at the inner radial bound of the region of interest and progresses radially outward as the parameters causing this breakdown are increased. This analysis will not deal with the region where the vortex breaks down; that is, the analysis will only be valid for the portion of the region of interest where the Inviscid radial velocity is directed toward the vortex axis.

The working fluid may enter the region of interest at the outer cyllnder by being injected tangentially through slot jets, by being blown through a rotating porous cylinder or by some equivalent method such that the velocity boundary conditions at the outer cylinder are:

$$
\text { At } r^{*}=R ; u^{*}=-V v^{*}=\lambda V \quad w^{*}=0
$$

where $\lambda$ is a positive constant representing the ratio of the inlet tangential velocity to the inlet radial velocity. These boundary conditions prescribe the driving force of the problem. 
It is assumed that the inner cylinder at $r^{*}=\varepsilon R$ marks the boundary of the region of interest and acts as one electrode for the electrical circuit but does not present an obstruction to the flow of the fluid. This assumption does not allow the prescription of velocities at the inner cylinder and therefore precludes consideration of a radial boundary layer on this cylinder. This assumption is compatible with the neglect of the second order radial derivatives in the Navier-Stokes equations in Chapter II. The analysis will not be concermed with the manner of exit of the flutd from the central core. The efection of fluld from the boundary layer which was discussed by Rosenzwelg, Lewellen and Ross [9] is assumed to occur in the central core $r^{*}<\varepsilon R$ and thus is not included in the present analysis.

B. The Electromagnetic Boundary Conditions

The applied magnetic field is in the axial direction and the fluid flow is primarily in the tangential direction, therefore an electromotive force is induced in the radial direction. For the device to operate as a power generator, the end plates must be Insulators and the cylindrical walls must be conducting electrodes connected to an external resistance.

For mathematical simplicity, assume that this external resistance consists of two flat plate resistors laminated onto the end plates. This model retains the symmetry about the plane $z^{*}=b / 2$ and also maintains the axial symmetry. Thus, in effect, radial sheet currents exist at $z^{*}$ equals 0 and $b$ in the mathematical 
model. This means that the tangentlal component of the magnetic field is discontinuous at $z^{*}=0$ and $b$. However, the radial and axial components of the magnetic field remain continuous at the boundaries because no other sheet currents exist.

The fact that the end plates are insulators requires that the axial component of the current is zero at $z^{*}=0$ and $b$. Thus At $z^{*}=0$ and $b ; B_{r}^{*}, B_{z}^{*}$, are continuous

$$
\mathrm{J}_{\mathrm{z}}^{*}=0
$$

The presence of axial currents within the cylindrical electrodes will again cause the tangential component of the magnetic fleld to be discontinuous but the other two components are still continuous. Further conditions at the cylindrical electrodes need not be stipulated since they are not necessary for the analysis. Thus

At $r^{*}=\varepsilon R$ and $R_{;} B_{r}^{*}, B_{Z}^{*}$ are continuous

As the distance from the device becomes very large, it is assumed that the electric and magnetic fields approach their applied values.

Thus

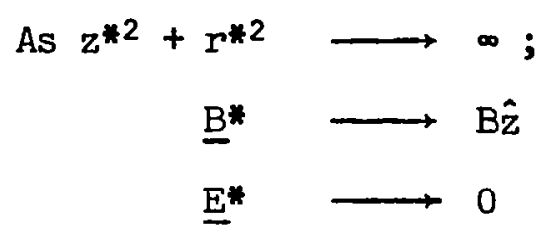




\section{The Temperature Boundary Conditions}

There are two possible forms for the temperature boundary conditions on the end"plates. The first is that there is no heat flow axially at the end plates. This condition may be expressed as:

(i) At $z^{\star}=0$ and $b ; \frac{\partial T^{*}}{\partial z^{\star}}=0$

The second condition is a prescribed end-wall temperature, it may be expressed as:

(11) At $z^{*}=0$ and $b ; \quad T^{*}=T_{w}$

At the outer cylindrical wall, it is assumed that the fluid enters the region of interest with a uniform prescribed value:

$$
\text { At } \quad \mathrm{r} *=\mathrm{R} ; \quad \mathrm{T} *=\mathrm{T}_{\mathrm{R}}
$$

In the development of the equations, the energy equation, like the Navier-Stokes' equations, will be simplified to parabolic form. Thus no temperature boundary conditions will be specified on the inner cylindrical wall. This agrees with the assumption that the inner cylindrical wall is not a real obstacle to the flow. 


\section{CHAPTER II}

\section{DEVETOPMENT OF EQUATIONS}

\section{Basic Equations}

The steady laminar motion of an incompressible electrically conducting fluid in the presence of a magnetic field is governed by the following equations:

Conservation of mass

$$
\mathrm{div}^{*} \underline{\mathrm{v}}^{*}=0
$$

Conservation of momentum

$$
\rho\left(\underline{v}^{*} \cdot \nabla\right) \underline{v}^{*}=-\operatorname{grad} p^{*}+\mu \nabla^{* 2} \underline{v}^{*}+\underline{J}^{*} \times \underline{B}^{*} \quad \text { II }-2
$$

Maxwell's equations

$$
\begin{array}{ll}
\operatorname{curl}^{*} \underline{B}^{*}=\mu_{m} \underline{J}^{*} & I I-3 \\
\operatorname{div}^{*} \underline{B}^{*}=0 & \text { II-4 } \\
\operatorname{curl}^{*} \underline{E}^{*}=0 & \text { II-5 } \\
\operatorname{dIv}^{*} \underline{E}^{*}=\rho_{e}^{*} / \varepsilon_{0} & I I-6
\end{array}
$$

Ohm's law

$$
\underline{J}^{*}=\sigma\left(\underline{E}^{*}+\underline{v}^{*} \times \underline{B}^{*}\right) \quad \text { II }-7
$$

Conservation of charge

$$
d \perp v^{*} \underline{J}^{*}=0
$$


Conservation of energy

$$
\rho c \frac{D T^{*}}{D t^{*}}=k \nabla * 2 T^{*}+\mu \phi^{*}+\frac{I}{\sigma} I^{*} \cdot I^{*} \quad I I-9
$$

where $\$$ is the viscous dissipation term and will be defined below. The asterisks are used to indicate dimensional variables.

\section{The Non-Dimensionalization}

The governing differential equations will be non-dimensionalIzed by choosing characteristic values of the variables appearing in the problem. When these governing equations are written out in cylindrical coordinates, the unknowns are functions of two spatial varlables, the radius $r^{*}$ and the axial dimension $z^{*}$. There is no dependence upon the azimuthal angle since the flow is assumed to be axisymmetric. The characteristic radial dimension is chosen as the radius of the outer cylinder, $R$, while the characteristic axial dimension is the separation distance between the two plates, b.

It is not obvious what the characteristic velocity should be. The tangential velocity $v^{*}$ is introduced into the region of interest with a different value than the radial velocity. However, postulation of differing characteristic velocities for these two velocity components only leads to a more complicated analysis and does not shed any light on the ordering procedure. Therefore, the tangential and radial velocities are given the same characteristic velocity. The value of the radial velocity at the outer cylinder, 
denoted by $v$, is chosen as the characteristic velocity. It is reasonable that the characteristic axial velocity be the same-as the characteristic radial velocity since the axial velocity is induced by conservation of mass to compensate for redistribution of the radial mass flow.

The pressure is nondimensionalfzed by a characteristic pressure P. This characteristic pressure $\$ 111$ be chosen such that the dimensionless pressure $p$ will be of unit order for all values of the variables, this choice is made in section 3 below. The characteristic temperature is chosen as the inlet fluid temperature $\mathrm{T}_{\mathbf{R}}$.

The characteristic value of the magnetic field is chosen as the value of the applied axial magnetic field, B. The electric field and electric current are induced by the ( $\underline{v} \times \underline{B}$ ) term appearIng in the Ohm's Law equation. Thus, obvious characteristic values for the electric field and current are VB and oVB respectively.

The dimensional variables may now be written as:

$$
\begin{aligned}
& r * \begin{array}{lll}
R r & \text { a } & T^{*}=T_{R} T \quad \text { e }
\end{array} \\
& z^{*}=\mathrm{bz} \quad \mathrm{b} \quad \underline{\mathrm{B}} * \mathbf{B} \underline{\mathrm{B}} \quad \mathrm{f} \\
& \underline{\mathbf{v}}^{*}=\underline{\mathrm{V}} \quad \mathrm{c} \quad \underline{E}^{*}=\mathrm{VBE} \quad \mathrm{g} \quad \text { II-10 } \\
& P^{*}=P_{p} \quad d \quad \underline{J^{*}}=\sigma \mathrm{VB} \underline{\mathrm{J}} \quad \mathrm{h} \\
& \rho_{\mathrm{e}}^{*}=\rho_{\mathrm{e}} \varepsilon_{0} \mathrm{VB} / \mathrm{R} \quad 1
\end{aligned}
$$

The governing equations II-1 through II-9 written in dimensionless form and in cylindrical coordinates are: 
$\frac{l}{r} \frac{\partial}{\partial r}(r u)+s \frac{\partial w}{\partial z}=0$

II-II

$u \frac{\partial u}{\partial r}+s w \frac{\partial u}{\partial z}-\frac{v^{2}}{r}=-E \frac{\partial p}{\partial r}+\operatorname{RmN}\left[J_{\phi} B_{z}-J_{z} B_{\phi}\right]$

$$
+\frac{1}{\operatorname{Re}}\left[\frac{\partial^{2} u}{\partial r^{2}}+\frac{\partial}{\partial r}\left(\frac{u}{r}\right)+s^{2} \frac{\partial^{2} u}{\partial z^{2}}\right]
$$

II-12

$u \frac{\partial v}{\partial r}+s w \frac{\partial v}{\partial z}+\frac{u v}{r}=+\operatorname{RmN}\left[J_{z} B_{r}-J_{r} B_{z}\right]$

$$
+\frac{1}{\operatorname{Re}}\left[\frac{\partial^{2} v}{\partial r^{2}}+\frac{\partial}{\partial r}\left(\frac{v}{r}\right)+s^{2} \frac{\partial^{2} v}{\partial z^{2}}\right]
$$

II-I3

$u \frac{\partial w}{\partial r}+s w \frac{\partial w}{\partial z}=-E s \frac{\partial p}{\partial z}+\operatorname{RmN}\left[J_{r} B_{\phi}-B_{r} J_{\phi}\right]$

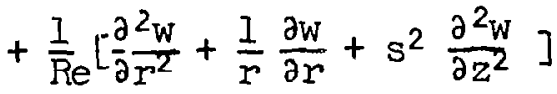

$$
\begin{aligned}
& -\frac{S}{\operatorname{Rm}} \frac{\partial B}{\partial z} \phi=J_{r} \\
& \frac{s}{\operatorname{Rm}} \frac{\partial B_{r}}{\partial z}-\frac{1}{\bar{R} m} \frac{\partial F_{z}}{\partial r}=J_{\dot{\varphi}} \\
& \frac{1}{\operatorname{R} m} \frac{1}{r} \frac{\partial}{\partial r}\left(E_{\phi} r\right)=J_{z} \\
& \frac{I}{r} \frac{\partial}{\partial r}\left(r B_{r}\right)+s \frac{\partial E}{\partial z} z=0 \\
& -\frac{\partial E}{\partial z} \phi=0 \\
& \mathrm{~s} \frac{\partial E_{r}}{\partial z}-\frac{\partial E}{\partial r} z=0
\end{aligned}
$$




$$
\begin{aligned}
& \frac{I}{r} \frac{\partial}{\partial r}\left(r E_{\phi}\right)=0 \\
& \frac{I}{r} \frac{\partial}{\partial r}\left(r E_{r}\right)+s \frac{\partial E}{\partial z} z=f_{e} \\
& J_{r}=E_{r}+v B_{z}-w B_{\phi} \\
& J_{\phi}=w B_{r}-U B_{z} \\
& J_{z}=E_{z}+u B_{\phi}-v B_{r} \\
& \frac{l}{r} \frac{\partial}{\partial r}\left(r J_{r}\right)+s \frac{\partial J_{z}}{\partial z}=0
\end{aligned}
$$

$u \frac{\partial T}{\partial r}+s w \frac{\partial T}{\partial z}=\frac{1}{\operatorname{RePr}}\left[\frac{\partial^{2} T}{\partial r^{2}}+\frac{1}{r} \frac{\partial T}{\partial r}+s^{2} \frac{\partial^{2} T}{\partial z^{2}}\right]$

$+\operatorname{RmN} E c\left[J_{I}^{2}+J_{\phi}^{2}+J_{z}^{2}\right]+\frac{E c}{R e}\left[\left(\frac{\partial u}{\partial r}\right)^{2}+\left(s \frac{\partial w}{\partial z}\right)^{2}\right.$

$$
\left.+\left(s \frac{\partial v}{\partial z}\right)^{2}+\left(\frac{\partial v}{\partial r}\right)^{2}+\left(s \frac{\partial u}{\partial z}+\frac{\partial w}{\partial r}\right)^{2}-\frac{v}{r} \frac{\partial v}{\partial r}\right]
$$

where $s$ is the shape parameter, Re is the Reynolds number, $E$ is the Euler number, $\mathrm{Rm}$ is the magnetic Reynolds number, $\mathrm{N}$ is the magnet1c pressure term, $\operatorname{Pr}$ is the PrandtI number and Ec is the Eckert number:

$\begin{array}{lllll}s=\mathrm{R} / \mathrm{b} & \mathrm{a} & \mathrm{N}=\mathrm{B}^{2} / \mu_{\mathrm{m}} \rho \mathrm{V}^{?} & \mathrm{e} & \\ \operatorname{Re}=\rho \mathrm{RV} / \mu & \mathrm{b} & \mathrm{Pr}=\mu \mathrm{c} / \mathrm{k} & \mathrm{f} & \mathrm{II}-28 \\ \mathrm{E}=\mathrm{P} / \rho V^{2} & \mathrm{c} & \mathrm{Ec}=\mathrm{V}^{2} / \mathrm{cT}_{\mathrm{R}} & \mathrm{g} & \\ \mathrm{Rm}=\mu_{\mathrm{m}} \sigma \mathrm{RV} & \mathrm{d} & & \end{array}$


Note that equations II-19 and 21 plus single valuedness of the potential yield

$$
\mathrm{E}_{\phi}=0
$$

The boundary conditions for the problem as described in section I are

$$
\begin{aligned}
& \text { At } \mathbf{z}=0 \text { and } 1 \quad u=v=w=0 \quad a, b, c \\
& \text { (i) } \mathrm{T}=\mathrm{Tw} / \mathrm{T}_{\mathrm{R}} \text { or } \quad \text { (ii) } \frac{\partial \mathrm{T}}{\partial \mathrm{z}}=0 \quad \mathrm{~d} \\
& \mathrm{~J}_{\mathrm{z}}=0 \\
& { }^{B}, B_{z} \quad \text { continuous } \quad f
\end{aligned}
$$

The physical configuration is symmetric about the plane $z=1 / 2$. The dependent variables are either symmetric or antisymmetric with respect to this plane of symmetry. For those which are symmetric, their first (and all other odd) derivatives are zero at $z=1 / 2$. For those which are antisymetric, the variables (and all even derivatives) are zero at $z=1 / 2$ :

$$
\begin{aligned}
& \text { At } z=1 / 2 \quad \frac{\partial}{\partial z}\left(\mathrm{u}, \mathrm{v}, \mathrm{T}, \mathrm{B}_{z}, \mathrm{E}_{r}, \mathrm{~J}_{r}, \mathrm{~J}_{\phi}\right)=0 \\
& \left(w, B_{r}, B_{\phi}, E_{z}, J_{z}\right)=0
\end{aligned}
$$

These symmetry conditions are not independent of conditions II-30 at the end plates but may partially replace them for convenience in determining the admissable forms of solutions for the variables.

The radial boundary condition is 
At $r=1$

$$
u=-1
$$

a

$\mathrm{v}=\lambda$

$\mathrm{b}$

$\mathrm{w}=0$

c

II-32

$T=I$

d

$\mathrm{B}_{r}, \mathrm{~B}_{z}$ continuous

e

The parameter $\lambda$ is the ratio of the inlet tangential velocity to the inlet radial velocity. It is expected that the term $\mathrm{VB}_{\mathrm{z}}$ in equation II-23 will govern the radial current flow and that $\mathrm{uB}_{\mathrm{z}}$ in equation II-24 will govern the tangential current. The rad1al current is the component which flows through the external circuit producing useful electric power while the tangential current is entirely dissipated as ohmic losses. For power applications, it is desirable to maximize the radial current and minimize the tangential current. This means that in a practical power generator $\lambda$ will be much larger than one. However, the subsequent analysis will not be restricted to this case but will retain $\lambda$ as a general constant.

The electromagnetic boundary conditions far from the device are:
At $z^{2}+r^{2}=\infty$
$\mathrm{B}_{\mathrm{r}}=0$
a
$\mathrm{B}_{\phi}=0$
$\mathrm{b}$
$\mathrm{B}_{\mathrm{z}}=1$
c
$\underline{E}=0$
a 


\section{Expansion in Powers of $1 / \mathrm{s}$}

With the energy equation being uncoupled, the flow pattern and electromagnetic field are functions of the coordinates $z$ and $r$, and of $s, R e, E, R m, N$ and $\lambda$, six dimensionless parameters. As the equations stand at the moment, their solution borders on the impossible; some simplification must be introduced. The usual method employed, and the one to be employed presently, is to assume that one of the dimensionless parameters has a value either much greater than or much less than unity. The terms of the equations are then ordered with respect to this parameter and only the dominant terms are retained.

The intent of this analysis is to investigate the influence of the end walls upon the flow pattern within the region of interest . Since this influence is most pronounced when the plates are relatively close together, and since there is a significant simpliflcation of the equations for this case, consideration will now be given to the case for which $s$ is much larger than one; that is, when the radius of the outer cylinder, $R$, is much larger than the separation distance between the two plates, $b$.

That is, consider now that

$$
s>>1
$$

The first step in the simplification of the equations is to expand the dependent variables in powers of $1 / \mathrm{s}$, a small quantity: 


$$
\begin{aligned}
& u=u_{0}+\frac{1}{s} u_{1}+\frac{1}{s} u_{2}+\ldots \quad a \\
& v=v_{0}+\frac{1}{s} v_{1}+\frac{1}{s^{2}} v_{2}+\ldots \quad b \\
& w=w_{0}+\frac{1}{s} w_{1}+\frac{1}{s^{2}} w_{2}+\ldots \quad c \\
& T=T_{0}+\frac{1}{s} T_{1}+\frac{1}{s^{2}} T_{2}+\ldots \quad d \\
& p=p_{0}+\frac{1}{s} p_{1}+\frac{1}{s^{2}} p_{2}+\ldots \quad e \\
& \underline{B}=\underline{B}_{0}+\frac{1}{s} \underline{B}_{1}+\frac{1}{s^{2}} \underline{B}_{2}+\ldots \quad f \\
& \underline{E}=\underline{E}_{0}+\frac{1}{s} \underline{E}_{1}+\frac{1}{s^{2}} \underline{E}_{2}+\ldots \quad g \\
& \underline{J}=\underline{J}_{0}+\frac{1}{s} \underline{J}_{1}+\frac{1}{s^{2}} \underline{J}_{2}+\ldots \quad h \\
& \rho_{e}=\rho_{e o}+\frac{1}{s} \rho_{e_{1}}+\frac{1}{s^{2}} \rho_{e_{2}}+\ldots 1
\end{aligned}
$$

\section{II-35}

Now the components $u_{i}, B_{r i}$, etc. are assumed to be of unit order for any $s \gg 1$. These components may not be completely independent of $s$; their shape (but not magnitude) may still depend on $s$.

The ordered components with subscript zero will satisfy the boundary conditions II-30 through II-33. All other components will satisfy analogous conditions but with boundary values of zero. Substitution of equations II-35 into equations II-1I through II-27 and grouping terms with equal powers of $s$ yields: $\frac{\partial w_{0}}{\partial z}+\frac{1}{s}\left[\frac{1}{r} \frac{\partial}{\partial r}\left(u_{0} r\right)+\frac{\partial w_{1}}{\partial z}\right]+\frac{1}{s}\left[\frac{1}{r} \frac{\partial}{\partial r}\left(u_{1} r\right)+\frac{\partial w_{2}}{\partial z}\right]+\ldots=0$ 
$E\left[\frac{d p}{d r}+\frac{l}{s} \frac{d p}{d r}+\ldots\right]+s w_{0} \frac{\partial u_{0}}{\partial z}+\left[u_{0} \frac{\partial u_{0}}{\partial r}+w_{1} \frac{\partial u_{0}}{\partial z}+w_{0} \frac{\partial u_{1}}{\partial z}-\frac{v_{0}^{2}}{r}\right]+\ldots$

$=\frac{\varepsilon^{2}}{\operatorname{Re}} \frac{\partial^{2} u_{0}}{\partial z^{2}}+\frac{\partial^{2} u_{1}}{\partial z^{2}}+\frac{I}{R e}\left[\frac{\partial^{2} u_{0}}{\partial r^{2}}+\frac{\partial}{\partial r^{2}}\left(\frac{u_{0}}{r}\right)+\frac{\partial^{2} u_{2}}{\partial z^{2}}\right]+\ldots$

II-37

$+\operatorname{RmN}\left[\mathrm{J}_{\phi O} \mathrm{~B}_{z 0}-\mathrm{J}_{z 0} \mathrm{~B}_{\phi O}\right]+\frac{R \mathrm{mN}}{\mathrm{s}}\left[\mathrm{J}_{\phi 1} \mathrm{~B}_{\mathrm{zO}}+\mathrm{J}_{\phi O} \mathrm{~B}_{\mathrm{zl}}-\mathrm{J}_{\mathrm{zl}} \mathrm{B}_{\phi O}\right.$

$$
\left.-J_{z 0} B_{\phi 1}\right]+\ldots
$$

$\mathrm{sw}_{0} \frac{\partial \mathrm{v}_{0}}{\partial z}+\left[\mathrm{u}_{0} \frac{\partial \mathrm{v}_{0}}{\partial \mathrm{r}}+\mathrm{w}_{1} \frac{\partial \mathrm{v}_{0}}{\partial z}+\mathrm{w}_{0} \frac{\partial \mathrm{v}_{1}}{\partial z}+\frac{\mathrm{u}_{0} \mathrm{v}_{0}}{\mathrm{r}}\right]+\ldots=$

$s^{2} / \operatorname{Re} \frac{\partial^{2} v_{0}}{\partial z^{2}}+\frac{s}{\operatorname{Re}} \frac{\partial^{2} v_{1}}{\partial z^{2}}+\frac{1}{\operatorname{Re}}\left[\frac{\partial^{2} v_{0}}{\partial r^{2}}+\frac{\partial}{\partial r}\left(\frac{v_{0}}{r}\right)+\frac{\partial^{2} v_{2}}{\partial z^{2}}\right]+\ldots \quad$ II -38

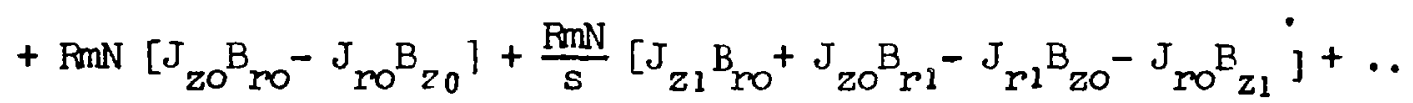

$s\left[w_{0}-\frac{\partial w_{0}}{\partial z}\right]+\left[u_{0} \frac{\partial w_{0}}{\partial r}+w_{1} \frac{\partial w_{0}}{\partial z}+w_{0} \frac{\partial w_{1}}{\partial z}\right]+\ldots+E\left[s \frac{\partial p_{0}}{\partial z}+\frac{\partial p_{1}}{\partial z}+\ldots\right]$

$=\frac{s^{2}}{\operatorname{Re}} \frac{\partial^{2} w_{0}}{\partial z^{2}}+\frac{s}{R e} \frac{\partial^{2} w_{1}}{\partial z^{2}}+\frac{1}{R e}\left[\frac{\partial^{2} w_{0}}{\partial r^{2}}+\frac{1}{r} \frac{\partial w_{0}}{\partial r}+\frac{\partial^{2} w_{2}}{\partial z^{2}}\right]+\ldots$

II-39

$+R m N\left[J_{10} B_{\phi 0}-J_{\phi 0} B_{r 0}\right]+\frac{R m N}{s}\left[J_{r_{1}} B_{\phi_{0}}+J_{r 0} B_{\phi 1}-J_{\phi 1} B_{r_{0}}-J_{\phi_{0}} B_{r_{1}}\right]+\ldots$

$-\frac{\mathrm{s}}{\mathrm{R}} \frac{\partial \mathrm{B}_{\phi 0}}{\partial z}-\frac{1}{\mathrm{R}} \frac{\partial B_{\phi 1}}{\partial z}+\ldots=J_{r 0}+\frac{1}{s} J_{r 1}+\ldots$

$\frac{\mathrm{s}}{\bar{m}} \frac{\partial \mathrm{B}_{r_{0}}}{\partial z}+\frac{1}{\bar{R} m}\left(\frac{\partial \mathrm{B}_{r_{1}}-\frac{\partial \mathrm{B}_{r_{0}}}{\partial z}}{\partial r}\right)+\ldots=\mathrm{J}_{\phi_{0}}+\frac{1}{\mathrm{~s}} \mathrm{~J}_{\phi \dot{\hat{I}}}+\ldots$

II-4I 
$\frac{1}{\mathrm{~F}} \frac{\partial}{\mathrm{r} \partial \mathrm{r}}\left(\mathrm{rB} \mathrm{B}_{\phi 0}\right)+\frac{1}{\mathrm{sFm}} \frac{1}{\mathrm{r} \partial \mathrm{r}}\left(\mathrm{rB}_{\phi 1}\right)+\ldots=\mathrm{J}_{\mathrm{z} 0}+\frac{1}{\mathrm{~s}} \mathrm{~J}_{\mathrm{z} 1}+\ldots \mathrm{II}-42$

$\frac{\partial B_{z 0}}{\partial z}+\frac{1}{s}\left[\frac{1}{r} \frac{\partial}{\partial r}\left(r B_{r 0}\right)+\frac{\partial B_{z 1}}{\partial z}\right]+\ldots=0$

$\frac{\partial E_{r 0}}{\partial z}+\frac{1}{s}\left[\frac{\partial E_{r 1}}{\partial z}-\frac{\partial E_{r 0}}{\partial r}\right]+\ldots=0$

$\frac{\partial E_{z 0}}{\partial z}+\frac{1}{s}\left[\frac{1}{r} \frac{\partial}{\partial r}\left(r E_{r 0}\right)+\frac{\partial E_{z_{1}}}{\partial z}\right]+\ldots=\rho_{e}$

$J_{r_{0}}-E_{r_{n}}+\frac{1}{s}\left(J_{r_{1}}-E_{r_{1}}\right)+\ldots=$

$v_{0} B_{z 0}-w_{0} B_{\phi 0}+\frac{1}{s}\left(v_{1} B_{z 0}+v_{0} B_{z 1}-w_{1} B_{\phi 0}-w_{1} B_{\phi 0}\right)+\ldots$

II-46

$J_{\phi 0}+\frac{l}{s} J_{\phi 1}+\ldots=w_{0} B_{r 0}-u_{0} B_{z 0}+$

$\frac{1}{s}\left(w_{1} B_{r 0}+w_{0} B_{r 1}-u_{1} B_{z 0}-u_{1} B_{z_{1}}\right)+\ldots$

II-47

$J_{z_{0}}-E_{z 0}+\frac{l}{s}\left(J_{z_{1}}-E_{z_{1}}\right)+\ldots=u_{0} B_{\phi_{0}}-v_{0} B_{r 0}+$

$\frac{I}{s}\left(u_{1} B_{\phi 0}+u_{0} B_{\phi 1}-v_{1} B_{r 0}-v_{0} B_{r 1}\right)+\ldots$

II-48

$\frac{\partial J_{z_{0}}}{\partial z}+\frac{1}{s}\left[\frac{l}{r} \frac{\partial}{\partial r}\left(r J_{r 0}\right)+\frac{\partial J_{z_{1}}}{\partial z}\right]+\ldots=0$

II-49 
$\mathbf{s w}_{0} \frac{\partial \mathrm{T}_{0}}{\partial \mathrm{z}}+\left[\mathbf{u}_{0} \frac{\partial \mathrm{T}_{0}}{\partial \mathrm{r}}+w_{1} \frac{\partial \mathrm{T}_{0}}{\partial \mathrm{z}}+w_{0} \frac{\partial \mathrm{T}_{1}}{\partial z}\right]+\ldots=$

$\frac{s^{2}}{\operatorname{RePr}} \frac{\partial^{2} \mathrm{~T}_{0}}{\partial z^{2}}+\frac{s}{\operatorname{RePr}} \frac{\partial^{2} \mathrm{~T}_{1}}{\partial z^{2}}+\frac{1}{\operatorname{RePr}}\left[\frac{\partial^{2} \mathrm{~T}_{0}}{\partial \mathrm{r}^{2}}+\frac{1}{r} \frac{\partial \mathrm{T}_{0}}{\partial r}+\frac{\partial^{2} \mathrm{~T}_{2}}{\partial \mathrm{z}^{2}}\right]+\ldots$

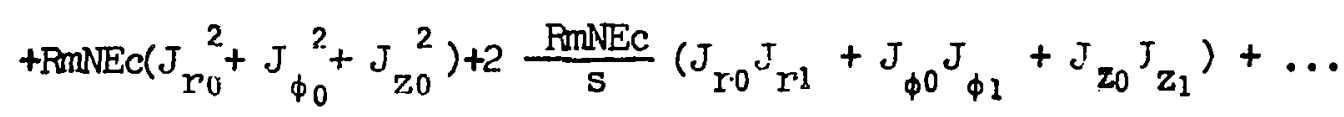

$+\frac{E c s^{2}}{\operatorname{Re}}\left[\left(\frac{\partial w_{0}}{\partial z}\right)^{2}+\left(\frac{\partial v_{0}}{\partial z}\right)^{2}+\left(\frac{\partial u_{0}}{\partial z}\right)^{2}\right]+$

II-50

$\frac{E c s}{R e} 2\left[\frac{\partial w_{0}}{\partial z} \frac{\partial w_{1}}{\partial z}+\frac{\partial v_{0}}{\partial z} \frac{\partial v_{1}}{\partial z}+\frac{\partial u_{0}}{\partial z} \frac{\partial u_{1}}{\partial z}+\frac{\partial u_{0}}{\partial z} \frac{\partial w_{0}}{\partial r}\right]+\ldots$

The method of solution of this set of equations II-36 through II-50 will be to solve for the zeroth order unknowns of several of these equations and use these results to simplify the remaining equations.

Consider first equations II-36, 43, 44, 45 and 49. Each of these equations contains only the dimensionless parameter $\mathrm{s}$ and the dominant term of each is a single variable differentiated with respect to $z$. The terms of these equations, and all the other equations, have been constructed to be of order one, independent of s. Thus in each of these equations, each set of terms multiplied by a different power of $s$ may be set equal to zero.

Thus the lead term of equation II-36 is

$$
\frac{\partial w_{0}}{\partial z}=0
$$


Inmeciate integration gives

$$
w_{0}=f_{1}(r)
$$

Applying the boundary condition II-30-c, the result is

$$
w_{0}=0
$$

II -51

This is an interesting result. In most analyses, it is assumed a priori that the terms of the continuity equation are of the same order. In the above analysis, this assumption was not made and the result is that the axial velocity is not of the same order as the other two velocity components; that is, while the radial and tangential velocities are of unit order, the axial velocity is of order $1 / \mathrm{s}$. It is physically reasonable that the axial velocity be small since it is prescribed zero on all boundaries where velocities are stipulated and since it has no primary driving force such as an imposed axial pressure gradient.

However, the fact that the zeroth order axial velocity $w_{0}$ is zero does not mean that all the inertla terms containing the axtal velocity can be automatically ignored. Inspection of the momentum equations II-37 and 38 and the energy equation II-50 reveals that the inertia and convection terms containing the first order axial velocity $w_{1}$ are of the same order as the inertia and convection terms containing the zeroth order radial and tangential velocities. This means that these inertia and convection terms containing $w_{1}$ are of importance when the other terms are and that 
the first order axial velocity $w_{1}$ must be considered along with the zeroth order radial and axial velocities.

Returning to the continuity equation II- 36 , it is seen that the terms multiplied by $(1 / \mathrm{s})$, when set equal to zero, form an equation for the zeroth order radial velocity $u_{0}$ and the first order axial velocity $w_{1}$. Since $w_{1}$ must be considered along with $u_{0}$ and $v_{0}$, this equation is of use in determining these unknowns and will be employed below.

Similarly, the lead term of equation II-43 yields, upon integration,

$$
B_{z 0}=f_{2}(r)
$$

The equations which govern the flow inside the device are satisfied for any $f_{2}(r)$. Outside the device $B_{z_{0}}$ satisfles Laplace's equation. It is known from the boundary conditions that $\mathrm{B}_{\mathrm{zo}}$ is continuous at the fluid-solid interface and is equal to one at

$$
z^{2}+r^{2}=\infty
$$

Thus the solution for $B_{z_{0}}$ outside the device is determinant for any $f_{2}(r)$; the function $f_{2}(r)$ may be chosen so as to simplify the subsequent analysis. The most convenient choice is $f_{2}(r)=1$. Thus

$$
\mathrm{B}_{\mathrm{z} 0}=1 \quad \mathrm{II}-52
$$

This means that, to the zeroth order in $1 / \mathrm{s}$, the axial magnetic field is undistorted by the presence of the two flat plates and the fluid contained between them. It is physically reasonable that if the plates are relatively close together, the distortion 
of the axial magnetic field is small. Note that equation II-52 has been obtained without Iimitation to small magnetic Reynolds number。

The zeroth order term of equation II-44 yields

$$
E_{r 0}=f_{3}(r)
$$

Again, as in the case of $\mathrm{B}_{\mathrm{z} 0}$, the equations within and outside of the device are valid and determinant for any value of $f_{3}(r)$ 。 However, in this case there is an additional restriction on the function $f_{3}(r) ; E_{r_{0}}$ must be such that Ohm's law for the extemal circuit is satisfled. Ohm's law states

$$
\Delta * * \tilde{I} * \tilde{R}
$$

where $\Delta \mathrm{E}^{*}$ is the potential difference applied to the external circuit, $\tilde{I}^{*}$ is the current flowing through that circuit and $\tilde{R}$ is the resistance of the extermal circuit.

In terms of the variables within the device,

$$
\begin{aligned}
& \tilde{I}^{*}=2 \pi R \sigma \mathrm{VBb} \int_{0}^{l} r J_{r} d z \\
& \tilde{\Delta E^{*}}=-\operatorname{VBR} \int_{\varepsilon}^{1} E_{Y} d r
\end{aligned}
$$

where the minus sign in equation II-56 is introduced to account for the fact that $E_{r}$ is an induced electric field and $\tilde{\Delta E}^{*}$ is an applied electric field.

Written in dimensionless form and retaining only the dominant terms in powers of $1 / \mathrm{s}$, equation II- 54 becomes 
$\int_{\varepsilon}^{l} f_{3}(r) d r=-2 \pi \sigma b R \int_{0}^{1} n r_{0} d z$

where equation II-53 has been used to replace $\mathrm{E}_{\mathrm{r}_{0}}$.

Thus it is obvious that $f_{3}(r)$ cannot be arbitrarily chosen as $f_{2}(r)$ was. Equation II-57 will be developed further when the ordering procedure ylelds further information concerning the relation between $f_{3}(r)$ and $J_{r_{0}}$.

Next, equation II-45 y1elds

$$
E_{z_{0}}=f_{4}(r)+\int^{z} \rho_{e o} d z
$$

The problem posesses symmetry about the plane $z=1 / 2$ and $E_{z}$ is antisymmetric. Thus, by equation II-31,

$$
E_{z_{0}}=\int_{\frac{1}{2}}^{z} \rho_{e o} d z \quad I I-58
$$

Lastly, equation II-49 yields

$$
J_{z_{0}}=f_{5}(r)
$$

or, since the end plates are insulators, if is obvious that

$$
J_{z^{0}}=0
$$

The zeroth order terms of equations II-36, 43, 44, 45 and 49 have been solved for $w_{0}, B_{z_{0}}, E_{r_{0}}, E_{z_{0}}$ and $J_{z_{0}}$ respectively. Equation II-45 ylelded a relation between $E_{z_{0}}$ and the still unknown $\mathrm{p}_{e}$. The remaining zeroth order unknowns, still undetermined, are $u_{0}, v_{0}$, $\mathrm{p}_{0}, \mathrm{~B}_{\mathrm{r} 0}, \mathrm{~B}_{\phi 0}, \mathrm{~J}_{\mathrm{r} 0}, \mathrm{~J}_{\phi_{0}}$ and $\mathrm{T}_{0}$. In order to solve the governing 
equations for these zeroth order unknowns, it is necessary to solve simultaneously the equations for one first order unknown, $w_{1}$ since it appears in the momentum, continuity and energy equations where $w_{0}$ would normally be expected to appear. For convenience, the set of unknowns $u_{0}, v_{0}, w_{1}, p_{0}, \underline{B}_{0}, \underline{E}_{0}$ and $\underline{J}_{0}$ will be referred to as the zeroth order set of unknowns.

The analysis will now be concerned with completing the determination of the zeroth order set of unknowns. For this purpose, equations II-36, 37, 38, 39, 40, 4I, 46, 47, 48 and 50 will be analyzed further. Equation II-42 will not enter into this consideration since, for general magnetic Reynolds number, its dominant terms involve a higher order unknown:

$$
\frac{s}{\mathrm{Rm}} \frac{I}{\mathrm{r}} \frac{\partial}{\partial \mathrm{r}}\left(\mathrm{rB}_{\phi 0}\right)=\mathrm{J}_{\mathrm{z} 1}
$$

For magnetic Reynolds number of order one or smaller, the solution for the tangential magnetic field $B_{\phi_{0}}$ found from equation II-40 will automatically satisfy equation II-42.

Note that the leading non-linear term in each of equations II-37, 38, and 39 disappears since $w_{0}=0$. Equations II-36, 37, $38,39,40,41,46,47,48$ and 50 may now be written with the higher order terms neglected.

$$
\frac{1}{r} \frac{\partial}{\partial r}\left(u_{0} r\right)+\frac{\partial w_{1}}{\partial z}=0
$$


$u_{0} \frac{\partial u_{0}}{\partial r}+w_{1} \frac{\partial u_{0}}{\partial z}-\frac{v_{0}^{2}}{r}+E \frac{\partial p_{0}}{\partial r}=\frac{s^{2}}{R e} \frac{\partial^{2} u_{0}}{\partial z^{2}}+R m N J_{\phi 0}$

$I I-6 I$

$u_{0} \frac{\partial v_{0}}{\partial r}+w_{1} \frac{\partial v_{0}}{\partial z}+\frac{u_{0} v_{0}}{r}=\frac{s^{2}}{R e} \frac{\partial^{2} v_{0}}{\partial z^{2}}-\operatorname{RmN} J_{r_{0}}$

$I I-62$

$s E \frac{\partial p_{\jmath}}{\partial z}=\frac{s}{R e} \frac{\partial^{2} w_{1}}{\partial z^{2}}+\operatorname{RnN}\left[J_{r 0} B_{\phi 0}-J_{\phi 0} B_{r 0}\right]$

$-\frac{s}{\bar{R} m} \frac{\partial \mathrm{B}_{\phi 0}}{\partial z}=\mathrm{J}_{r_{0}}$

II-64

$\frac{s}{\bar{m}} \frac{\partial B}{\partial z}=J_{\$ 0}$

II-65

$$
J_{r^{0}}=v_{0}+f_{3}(r)
$$

$$
J_{\phi 0}=-u_{0}
$$

$$
0=u_{0} B_{\phi_{0}}-v_{0} B_{r 0}+\int_{\frac{1}{2}}^{z} \rho_{e} d z
$$

$u_{0} \frac{\partial T_{0}}{\partial r}+w_{1} \frac{\partial T_{0}}{\partial z}=\frac{s^{2}}{\operatorname{RePr}} \frac{\partial T_{0}}{\partial z^{2}}+\operatorname{RnN} \operatorname{Ec}\left(J_{r_{0}}{ }^{2}+J_{\phi 0}{ }^{2}\right)$

$$
+\operatorname{Ec} \frac{s^{2}}{\operatorname{Re}}\left[\left(\frac{\partial v_{0}}{\partial z}\right)^{2}+\left(\frac{\partial \lambda_{0}}{\partial z}\right)^{2}\right]
$$

The axial momentum equation II-63 may be directly simplified by consideration of the Euler number $E=P / \rho V^{2}$. In the non-dimensionalization of the pressure in equation II-10, the characteristic pressure $P$ was chosen such that the dimensionless pressure $p$ would be unit order for all ranges of parameters considered. 
Consider the radial momentum equation II-6I. The pressure term is the driving force in that equation; if there is no radial pressure gradient, the radial velocity becomes zero. Therefore, the term ( $\mathrm{E} \frac{\hat{\partial} \mathrm{p}_{0}}{\partial \mathrm{r}}$ ) must be as large as the largest of the remaining terms in the radial momentum equation II-61. Since all the var1ables have been non-dimensionalized to be of unit order, this is equivalent to saying that the Euler number, $E$, must be equal to the largest of $1, s^{2} /$ Re and $\mathrm{RnN}$.

Thus

$$
\mathrm{E} \geq \frac{\mathrm{s}^{2}}{\operatorname{Re}} ; \mathrm{E} \geq \mathrm{RmN} ; \mathrm{E} \geq 1 \quad \text { II-70 }
$$

Now consider the axial momentum equation II-63 in the light of equation II-70. Comparison of the pressure term with the magnet1c term in equation II-63 shows that the pressure term is always at least of order $s$ larger than the magnetic term. Therefore, the magnetic term in the axial momentum equation may be neglected.

By a similar comparison, it is seen that the pressure term Is always at least of order $s^{2}$ larger than the viscous term in the axial momentum equation even within a boundary layer. Therefore, the viscous term may also be neglected and equation II-63 is reduced to

$$
\frac{\partial p_{0}}{\partial z}=0 \quad \text { II } 71
$$


This result is also obtained in a single plate analysis by assuming a large Reynolds number. In this case it was obtained as a result of the geometric restriction to large radius compared with separation distance; there is no restriction upon the size of the Reynolds number.

Equation II-71 stating that the zeroth order pressure is independent of the axial coordinate, was gained by neglecting the second order axial derivatives in the axial momentum equation. Now the governing equations only contain a first order axial derIvative of the axtal velocity.

In the single plate problem, this neglect caused no difficulty since there was only one axial boundary condition specifled on the axial velocity, $w(r, 0)=0$. The axial velocity far from the plate was not specified.

Now let a second plate be introduced to confine the vortex. This plate has two effects upon the conditions of the flow. The first is that it requires a second axial boundary condition for the axial velocity on the form of $w(r, 1)=0$. The second effect is that the radial pressure gradient $d p / d r(r)$, which was known in the single plate problem, is now an unknown.

The addition of the second plate to the flow picture adds one condition $w(r, I)=0$ and one unknown $d p / d r$. It is apparent that these two effects are inter-related. The presence of the second plate, causing the no flow boundary condition, requires 
that the radial pressure gradient be just that unique value which will allow the no flow condition to be satisfied. In actuality, this second axial boundary condition on the axial velocity is used to determine the radial pressure gradient. This will be explatned in detail after the governing equations are further simplified.

Equation II-66 may be combined with equation II-57 to yield an expression for $f_{3}(r)$ in terms of the tangential velocity $v_{0}$ :

$$
f_{3}(r)=\frac{\lambda a}{r}-\int_{0}^{1} v_{0} d z \quad I I-72
$$

where

$$
\lambda a \equiv \frac{\int_{0}^{1} \int_{\varepsilon}^{1} v_{0} d r d z}{2 \pi \sigma \hat{R} b+\ln \frac{1}{\varepsilon}}
$$

Now the radial current may be expressed as

$$
J_{r 0}=\frac{\lambda \alpha}{r}+v_{0}-\int_{0}^{1} v_{0} d z
$$

where the term ad represents that portion of the radial electric current which passes through the external circuit to produce useful electric power and the term $\left(v_{0}-\int_{0}^{1} v_{0} d z\right)$ represents the eddy currents caused by the variation of the tangential velocity profile with $z$.

Since the radial and tangential currents are known entirely In terms of velocities by equations II-67 and II-74, equations II-60,61, 62, and 69 may be written completely in terms of velocitles rather than contain any electric current terms: 
$\frac{l}{r} \frac{\partial}{\partial r}\left(r u_{0}\right)+\frac{\partial w_{1}}{\partial z}=0$

$u_{0} \frac{\partial u_{0}}{\partial r}+w_{2} \frac{\partial u_{0}}{\partial z}-\frac{v_{0}^{2}}{r}+E \frac{d p_{0}}{d r}=\frac{s^{2}}{R e} \frac{\partial^{2} u_{0}}{\partial z}-E m N u_{0}$

II-76

$u_{0} \frac{\partial v_{0}}{\partial r}+w_{1} \frac{\partial v_{0}}{\partial z}+\frac{u_{0} v_{0}}{r}=\frac{s^{2}}{R e} \frac{\partial^{2} v_{0}}{\partial z^{2}}-\operatorname{RnN}\left(\frac{\alpha \lambda}{r}+v_{0}-\int_{0}^{1} v_{0} d z\right)$

II-77

$u_{0} \frac{\partial T_{0}}{\partial r}+w_{1} \frac{\partial T_{0}}{\partial z}=\frac{s^{2}}{R e P r} \frac{\partial^{2} T_{0}}{\partial z^{2}}+E c \frac{s^{2}}{R e}\left[\left(\frac{\partial v_{0}}{\partial z}\right)^{2}+\left(\frac{\partial u_{0}}{\partial z}\right)^{2}\right]$

$+\operatorname{RmN} \operatorname{Ec}\left[u_{0}^{2}+\left(\frac{\lambda \alpha}{r}+v_{0}-\int_{0}^{1} v_{0} d z\right)^{2}\right]$

The applicable boundary conditions are II-30-a,b,c,d; II-3I; II $-32-a, b, c, d$.

Equations II-75 through II-78 do not contain any electromagnetic variables; they may be solved without recourse to equations II-64 through II-68. After the velocity profiles are determined, equations II-74 and II-67 yield the electric current components ${ }^{\top} r_{0}$ and $J_{\phi 0}$ respectively. Next, equations II-64 and 65 may be solved for $\mathrm{B}_{\phi 0}$ and $\mathrm{B}_{\mathrm{r} 0}$. Finally, equation II-68 may be differentiated and solved for the unknown electric charge density $p_{e}$

There are two axial boundary conditions to be satisfied by the axial velocity:

At $z=0$ and $z=1$

$w_{1}=0$

II $-30-c$ 
One of these two conditions may be satisfied as a normal boundary condition on $w_{1}$. The other condition may be considered as a fourth equation, to be combined with the three governing equations II-75, 76 and 77 , to be solved for the four unknowns $u_{0}, v_{0}, w_{1}$, and $E d p_{0} / d r$.

In theory, the set of equations II- 75,76 and 77 may be solved for the velocity components $u_{0}, v_{0}$, and $w_{1}$ in terms of the governIng parameters $\lambda, s^{2} / \operatorname{Re}$ and $\mathrm{RmN}$ and also in terms of the pressure gradient $E d p_{0} / d r$. This radial pressure gradient is not yet known. In general, these velocity solutions will not be able to satisfy both of the conditions II-30-c on the axial velocity since only the first derivative of the axial velocity appears in the governing equations. However, there exists a particular, unlque radial pressure gradient $\left(E d p_{0} / d r\right)$ for which both of these conditions can be satisfied. This is the pressure gradient which will exist naturally between the two flat plates.

This pressure gradient may be determined as follows: First integrate the continuity equation II-75 with respect to $z$ from $z=0$ to $z=1$. Application of the boundary conditions II-30-c yields

$$
\int_{0}^{1} \frac{1}{r} \frac{\partial}{\partial r}\left(u_{0} r\right) d z=0
$$

Since the limits of integration are independent of the radius $r$, equations II-79 may be integrated to yield 


$$
\int_{0}^{1} u_{0} d z=\frac{c}{r}
$$

The boundary condition $u_{0}(I, z)=-1$ gives finally

$$
\int_{0}^{1} u_{0} d z=-\frac{1}{r}
$$

This is an integral representation of the coriservation of mass equation. It states that the total amount of radial mass flow at any radial station is a constant (equal to -1 ). Thus, equations II-75, 76 and 77 may be solved for $u_{0}, v_{0}$, and $w_{1}$ in terms of a general $\left(E \frac{d p_{0}}{d r}\right)$. The solution for the radial velocity

$$
u_{0}=u_{0}\left(r, z ; R n N, \frac{s^{2}}{R e}, \lambda, E \frac{d p_{0}}{d r}\right)
$$

may be substituted into equation II-80 to yield a solution for the radial pressure gradient

$$
E \frac{d p_{0}}{d r}=E \frac{d p_{0}}{d r}\left(r ; R m N, \lambda, \frac{s^{2}}{R e}\right)
$$

With $u_{0}$ known in terms of this particular pressure gradient, the continuity equation II-75 yields a solution for $w_{1}$ which satisfies both axial boundary conditions II-30-c.

\section{A Discussion of Types of Flow}

The type of flow existing in the region of interest as represented by equations II-75, 76 and 77 depends upon the values of 
the dimensionless groups appearing in these equations, $s^{2} / \mathrm{Re}, \mathrm{FmN}$

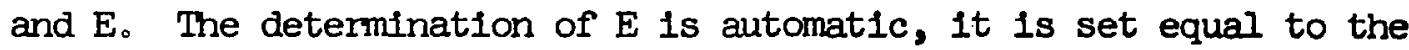
largest of $1, \mathrm{~s}^{2} / \mathrm{Re}$ and $\mathrm{RmN}$. Therefore, there are only two Independent dimenstonless groups governing the type of flow. In the followIng, RmN and $s^{2} /$ Re will be assumed small (of order $1 / s$ or smaller), of unit order, or large (of order $s$ or larger) and the resulting flow patterns will be brlefly discussed.

CASE A

Consider first the case of $\mathrm{RmN}$ negligible; the Reynolds number is ordered below. This may be caused physically by a weak magnetic field or small electric conductivity. Since the governing equations have been developed by neglecting terms of order $1 / \mathrm{s}$ compared with the dominant terms, this is equivalent to considering $\mathrm{RmN} \leq 1 / \mathrm{s}$

In this case, the magnetic terms in equations II-76 and 77 are small compared with the inertia terms and may be neglected. This removes any effect that the magnetic field has on the flow; the flow is hydrodynamic to order $1 / \mathrm{s}$.

A 1

Consider the $s^{2} / R e$ is small in addition to $\mathrm{RmN}$ :

$\operatorname{RmN} \leq 1 / \mathrm{s}$ and $\mathrm{s}^{2} / \operatorname{Re} \leq 1 / \mathrm{s}$

It follows from equation II-70 that $E=1$. The inertia terms dominate the viscous terms except in two boundary layer regions near the two end plates. That is, equations II-75, 76 and 77 with 
the terms on the right hand side of the equal signs replaced by zero approximately govern the flow in the central region between the two plates. The flow in the two boundary layer regions lying between this central region and the two plates is governed by equations II-75, 76 and 77 without the magnetic terms but with the viscous terms present since the second order axial derivatives must be retained to satisfy the boundary conditions on the plates. This case was analyzed by Loper [8] using a momentum integral analysis. With the introduction of the parameter $s$, the normal role of the Reynolds number Re is replaced by a modified Reynolds number $\mathrm{Re} / \mathrm{s}^{2}$. The boundary layer thickness is of order $\sqrt{\mathrm{s}^{2} / \mathrm{Re}}$ rather than $\sqrt{l / R e}$.

A 2

Now consider $s^{2} /$ Re is of unit order (this is equivalent to Re >> 1) while RmN remains small:

$$
\mathrm{RmN} \leq \frac{1}{\mathrm{~s}} \quad \mathrm{~s}^{2} / \mathrm{Re}=0(1)
$$

Again it follows that $E=1$. In this case the viscous terms are of the same order as the inertia terms and viscous flow fills the region of interest; there is tangential flow throughout the region but there is no central core of inviscid flow.

A 3

Let $\mathrm{s}^{2} /$ Re be large while $\mathrm{RmN}$ is still small:

$$
\mathrm{RmN} \leq \frac{1}{\mathrm{~s}} \quad \mathrm{~s}^{2} / \mathrm{Re} \geq \mathrm{s}
$$


Now $E=s^{2} / R e$. The viscous terms dominate and a Stokes type of flow results from equations II-75, 76 and 77 . The tangential velocity has a non-zero value only in a boundary layer region near the outer cylinder. The radial velocity is non-zero throughout the region because of radial mass flow conservation; it assumes a parabolic profile away from the outer cylinder (see Appendix III for details). It is no longer possible to satisfy the boundary condition at the outer cylinder in this special case.

CASE B

Consider now that the magnetic parameter is of unit order: $\operatorname{RmN}=0(1)$

In this case the magnetic terms are of the same order as the inertia terms and must be considered whenever the inertia terms are. B 1

$$
\mathrm{RmN}=0(1) \quad \mathrm{s}^{2} / \mathrm{Re} \leq \frac{1}{\mathrm{~s}} \quad \mathrm{E}=1
$$

This case is very similar to case Al; there is a boundary layer flow but now the magnetic terms must be included. At this point it is not clear what influence the presence of the magnetic terms have on the boundary layer growth and boundary layer blockage. Chapter III, which is an approximate analysis of these cases, will describe this influence. 
B 2

$$
\mathrm{RmN}=0(1) \quad \mathrm{s}^{2} / \mathrm{Re}=0(1) \quad \mathrm{E}=1
$$

All dimensionless groups are of order one. This case is similar to A2; there is viscous flow throughout the region but with the magnetic terms present.

B 3

$$
\operatorname{RmN}=O(1) \quad s^{2} / \operatorname{Re} \geq s \quad E=s^{2} / \operatorname{Re}
$$

This case is identical to case A3. The viscous terms dominate the inertia and magnetic terms.

CASE C

Let the magnetic parameter be large

$$
\mathrm{RmN} \geq \mathrm{s}
$$

The magnetic terms are larger than the inertia terms; the latter may be neglected. With the neglect of the inertia terms it becomes impossible to satisfy the radial boundary condition at the outer cylinder since the radial derivative terms are ignored. In actuality radial boundary layers exist on the outer cylinder. In this way the radial boundary conditions are satisfied. The further ordering of $s^{2} /$ Re must now be done with respect to $\mathrm{RmN}$ rather than with respect to unity. 
C 1

$$
\mathrm{RmN} \geq \mathrm{s} \quad \mathrm{s}^{2} / \mathrm{Re} \leq \frac{\mathrm{RmN}}{\mathrm{s}} \quad \mathrm{E}=\mathrm{RmN}
$$

In this case the magnetic terms apparently dominate all others excepting the pressure term and is the governing term in the central region of the device. Near the boundarles the viscous terms become important and boundary layers occur. The inertia terms remain negligible.

C 2

$$
\mathrm{RmN} \geq \mathrm{s} \quad \mathrm{s}^{2} / \mathrm{Re}=O(\mathrm{RmN}) \quad \mathrm{E}=\mathrm{RmN}
$$

This case is analogous to case A2 but now the viscous terms balance the magnetic terms throughout the region of interest but the inertia terms remain negligible.

C 3

$$
\mathrm{RmN} \geq \mathrm{s} \quad \mathrm{s}^{2} / \mathrm{Re} \geq \mathrm{RmNs} \quad \mathrm{E}=\mathrm{s}^{2} / \mathrm{Re}
$$

This case is identical to cases B3 and A3. It is purely a Stokes type of flow with the magnetic terms as well as the inertia terms being negligible. 
CHAPTER III

EXACT SOLUTION OF THE LINEARTZED

MOMENTUM EQUATIONS

\section{The Linearization}

In this chapter, the set of equations II $-75,76$ and 77 are to be solved to yield the velocity profiles and the radial pressure gradient. The most common method of attacking such a coupled set of non-linear partial differential equations is that of momentum integral analysis. However, for this problem of a confined magneto hydrodynamic vortex flow, that approximate method of analysis is too cumbersome to be employed. Some other method of attack is needed. It is desired that this method yield a more exact solution and more details of the flow than the momentum integral method can.

Such a method which has been used with some success on nonlinear equations of this sort is the so called Oseen or Targ Inearization [10]. This linearization scheme is valid for general values of the governing parameters. This is the method of the solution which will be employed in this chapter.

The governing equations are 
$\frac{1}{r} \frac{\partial}{\partial r}(r u)+\frac{\partial w}{\partial z}=0$

$\left(u \frac{\partial}{\partial r}+w \frac{\partial}{\partial z}\right) u-\frac{v^{2}}{r}+E \frac{d p}{d r}=\frac{s^{2}}{R e} \frac{\partial^{2} u}{\partial z^{2}}-R m N u \quad I I-76$

$\left(u \frac{\partial}{\partial r}+w \frac{\partial}{\partial z}+\frac{u}{r}\right) v=\frac{s^{2}}{\operatorname{Re}} \frac{\partial^{2} v}{\partial z^{2}}-\operatorname{RmN}\left(\frac{\lambda \alpha}{r}+v-\int_{0}^{l} v d z\right) \quad$ II-77

where

$$
\lambda \alpha=\frac{\int_{\varepsilon}^{1} \int_{0}^{l} v d z d r}{2 \pi \sigma R \hat{~} b+\ln \frac{I}{\varepsilon}}
$$

The subscripts have been dropped from the unknowns for convenience; only the zeroth order set of unknowns will be considered below. The boundary conditions to be satisfied are given by equations II-30-a,b,c; II-3]; II-32-a,b,c。

Equation II-75 is already linear and need not be altered. Equation II-76 contains the non-Iinear operator ( $u \frac{\partial}{\partial r}+w \frac{\partial}{\partial z}$ ) as well as the non-linear term $\left(v^{2} / r\right)$. Both of these expressions will be linearized so that a tractable solution may be found. Similar$1 y$, equation II-77 contalns the non-linear operator $\left(u \frac{\partial}{\partial r}+w \frac{\partial}{\partial z}\right.$ $+u / r$ ) which also will be linearized.

In the linearization process, the varlables $u$ and $w$ appearIng in the non-linear operators are to be replaced by known functions which approximate these unknowns. There is a considerable simplification in the subsequent analysis if these replacement functions are purely radial functions. Therefore, let the radial velocity $u$ be replaced by some $g_{l}(r)$ and the axial velocity 
$w$ be replaced by some $g_{2}(r)$ in the non-linear operators where these functions $g_{1}$ and $g_{2}$ represent the average values of $u$ and $w$ respectively within the region of interest:

$$
\begin{aligned}
& u \longrightarrow \mathrm{g}_{1}(r) \\
& \mathrm{w} \longrightarrow \mathrm{g}_{2}(r)
\end{aligned}
$$

These functions $g_{1}(r)$ and $g_{2}(r)$ may be represented as the average of their respective functions over $z$ :

$$
\begin{aligned}
& g_{1}(r)=\int_{0}^{1} u d z \\
& g_{2}(r)=\int_{0}^{1} w d z
\end{aligned}
$$

The conservation of mass equation II-80 yields the result for $g_{1}$,

$$
g_{1}(r)=-1 / r
$$

It has been noted that the function $w$ is antisymetric with respect to the midplane $z=1 / 2$. Therefore, its integral from $z=0$ to $z=1$ is identically zero and

$$
g_{2}(r)=0
$$

Thus the operators become

$$
\begin{aligned}
& \left(u \frac{\partial}{\partial r}+w \frac{\partial}{\partial z}\right) \longrightarrow\left(-\frac{1}{r} \frac{\partial}{\partial r}\right) \\
& \left(u \frac{\partial}{\partial r}+w \frac{\partial}{\partial z}+\frac{u}{r}\right) \rightarrow\left(-\frac{1}{r} \frac{\partial}{\partial r}-\frac{1}{r^{2}}\right)
\end{aligned}
$$

This linearization scheme has several fortunate consequences beyond linearization of the equations. The first is that now only the even derivatives of $z$ appear in the mementum equations; 
they may be solved by known transform methods. The second consequence of the linearization is that the equations are uncoupled. The tangential momentum equation may first be solved for $v$. Next the radial momentum equation may be solved for $u$ in terms of the known $v$ and the unknown $E \frac{d p}{d r}$ This result for $u$ in terms of $\mathrm{E}$ dp/or may be substituted into the conservation of mass equation II-80; this yields the solution for the radial pressure term, $E d p / d r$. Finally the continuity equation II-75 yields the solution for the axial velocity $w$.

In order that the solution for the radial velocity $u$ may be found in terms of a simple Fourier series, the non=linear term $\left(\mathrm{v}^{2} / r\right)$ appearing in the radial momentum equation will be linearized in a manner similar to that above. One of the factors $v$ of this non-Iinear term will be approximated by its inviscid value $\lambda / r\left(1-\mathrm{RmN \alpha} \frac{1-r^{2}}{\text { ? }}\right)$. This value is given in Appendix III-Special Case Solutions in Eq. A-19. This Iinearization is verry good for large Reynolds numbers such that the boundary layers are small. For smaller Reynolds numbers it is more approximate but remains as accurate as the linearization process above. Thus

$$
\left(-\frac{\mathrm{v}^{2}}{\mathrm{r}}\right) \longrightarrow\left(-\frac{\lambda}{\mathrm{r}}\left(I-\mathrm{RmNa} \frac{I-\mathrm{r}^{2}}{2}\right) \frac{\mathrm{v}}{\mathrm{r}}\right) \quad \text { III-9 }
$$

Equations II-76 and 77 in linearized form are:

$-\frac{1}{r} \frac{\partial u}{\partial r}-\frac{\lambda v}{r^{2}}\left(1-R m N \alpha \frac{I-r^{2}}{2}\right)+E \frac{d p}{d r}=\frac{s^{2}}{R e} \frac{\partial^{2} u}{\partial z^{2}},-R m N u \quad$ III-10 
$-\frac{1}{r} \frac{\partial v}{\partial r}-\frac{v}{r} 2=\frac{s^{2}}{\operatorname{Re}} \frac{\partial^{2} v}{\partial r^{2}}-\operatorname{Rn} N\left(\frac{\lambda \alpha}{r}+v-\int_{0}^{1} v d z\right)$

\section{2: Solution of Linearized Tangential}

\section{Momentum Equation}

The tangential momentum Equation III-II must be solved before solution of the radial momentum equation III-10 is attempted since the tangential velocity $\mathrm{v}$ appears as a non-homogeneous term in equation III-10. Equation III-II is Iinear in the tangential velocity $\mathrm{v}$ since $\lambda \alpha$ is linear in $\mathrm{v}$. Equation III-II is a integrodifferential equation; it may be split up so that the differential and integral parts can be solved separately. In order to attack the differential form of equation III-Il first, replace the integral terms by the symbol $\lambda F(r)$ :

$$
\lambda F(r) \equiv \int_{0}^{l} v d z-\frac{1}{r} \frac{\int_{\varepsilon}^{l} \int_{0}^{l} v d z d r}{2 \pi \sigma \tilde{R b}+\ln \frac{1}{\varepsilon}}
$$

The term $\lambda$ is introduced into the definition of $F(r)$ so that it may be determined independentIy of $\lambda$.

With this substitution equation III-II is now

$$
-\frac{1}{r} \frac{\partial v}{\partial r}-\frac{v}{r^{2}}=\frac{s^{2}}{\operatorname{Re}} \frac{\partial^{2} v}{\partial z^{2}}-\operatorname{Rm} N(v-\lambda F(r))
$$

This tangential momentum equation may be solved by the finite sine transform method. The finite sine transform and its inverse are defined as [II] 


$$
\begin{aligned}
& \bar{v}_{n}(r, n) \equiv \pi \int_{0}^{l} v(r, z) \sin \pi n z d z \\
& v(r, z) \equiv \frac{2}{\pi} \sum_{n=1}^{\infty} \bar{v}_{n}(r, n) \sin \pi n z
\end{aligned}
$$

The problem under consideration posesses symmetry about the mid plane $z=1 / 2$.

Thus

$$
\frac{\partial v}{\partial z}(r, 1 / 2)=0
$$

This condition may be used to simplify the transform equations III-14 and 15. Applying this symmetry condition to equation III-15 yields

$$
\bar{v}_{n}(r, n)=0 \quad \text { for } n \text { even }
$$

Thus only terms with $\mathrm{n}$ odd need be considered; equations III-14 and 15 may be written

$$
\begin{aligned}
& \bar{v}_{2 n-1}(r, n)=\pi \int_{0}^{1} v(r, z) \sin \pi(2 n-1) z d z \\
& v(r, z)=\frac{2}{\pi} \sum_{n=1}^{\infty} \bar{v}_{2 n-1}(r, n) \sin (2 n-1) \pi z
\end{aligned}
$$

The transform of the second axial derivative may be written as

$$
\frac{\partial^{2} \bar{v}_{2 n-1}}{\partial z^{2}}=-(2 n-1)^{2} \quad \pi^{2} \bar{v}_{2 n-1}
$$

since $v(r, 0)=0$ and $v(r, l)=0$

Equation III-13 may be transformed using equation III-17 to 
the form

$$
-\frac{1}{r} \frac{d \bar{v}_{2 n-1}}{d r}-\frac{\bar{v}_{2 n-1}}{r^{2}}=-G_{n} \bar{v}_{2 n-1}+\frac{2 \lambda R m N}{2 n-1} F(r) \quad \text { III-20 }
$$

where

$$
G_{n} \equiv\left(\pi^{2} s^{2} / \mathrm{Re}\right)(2 n-1)^{2}+\mathrm{RmN}
$$

The solution of the non homogeneous linear ordinary differential equation III-20 may be easily found by the method of variation of parameters and is

$$
\bar{v}_{2 n-1}(r, n)=c_{n} \frac{1}{r} e^{G_{n} r^{2} / 2}-\frac{2 \lambda \operatorname{RmN}}{2 n-1} \frac{1}{r} \int^{r} n^{2} F(n) e^{G_{n} \frac{r^{2}-n^{2}}{2}} d n \quad \text { III-22 }
$$

The transform of the radial boundary condition II-32-b is

$$
\text { At } r=1 \quad \bar{v}_{2 n-1}=\frac{2 \lambda}{2 n-1}
$$

This condition allows the constant $C_{n}$ to be evaluated and yields for $\overline{\mathrm{v}}_{2 \mathrm{n}-\mathrm{I}}$ :

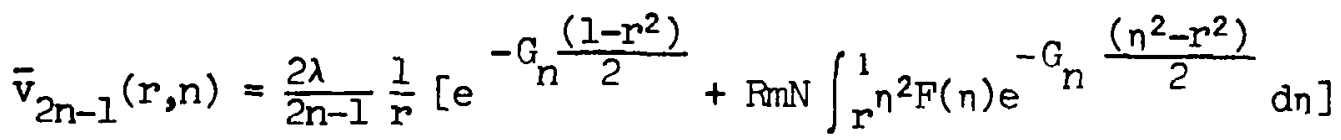

The inverse transform yields the result for the tangential velocity in terms of the function $F(r)$ which has not yet been determined.

$$
\begin{aligned}
& v(r, z)=\frac{4}{\pi} \frac{\lambda}{r} \sum_{n=1}^{\infty} \frac{\sin (2 n-1) \pi z}{2 n-1}\left\{e^{-G \frac{\left(1-r^{2}\right)}{2}}\right. \\
& \left.+R m N \int_{r}^{1} n^{2} F(n) e^{-G \frac{\left(n^{2}-r^{2}\right)}{2}} d n\right\}
\end{aligned}
$$


This completes the solution of the differential portion of the integro-differential equation III-1l。 Now the function $\lambda F(r)$, defined by equation III-12, will be determined. F1rst, the integrals of the tangential velocity $v$ with respect to $z$ and with respect, to both $z$ and $r$ must be calculated:

$\int_{0}^{1} v(r, z) d z=\frac{8}{\pi^{2}} \frac{\lambda}{r}\left[\sum_{n=1}^{\infty} \frac{1}{(2 n-1)^{2}} e^{-G_{n} \frac{\left(1-r^{2}\right)}{2}}+\right.$

$\left.\operatorname{RmN} \int_{r}^{1} n^{2} F(n) \sum_{n=1}^{\infty} \frac{1}{(2 n-1)^{2}} e^{-G n \frac{\left(n^{2}-r^{2}\right)}{2}} d n\right]$

Note that the infinite series in $\mathrm{n}$ appearing in equation III-2.6 are of the form

$$
e^{-R m N} \frac{\left(1-r^{2}\right)}{2} \sum_{n=1}^{\infty} \frac{1}{(2 n-1)^{2}} e^{-M(2 n-1)^{2}}
$$

where $M$ is a non-negative number. This series is highly convergent since

$$
\sum_{n=1}^{\infty} \frac{1}{(2 n-1)^{2}} e^{-M(2 n-1)^{2}} \leq \pi^{2} / 8
$$

for $M \geq 0$ 。

The double integral of $\mathrm{v}$ is

$\int_{\varepsilon}^{1} \int_{0}^{l} v(r, z) d r d z=\frac{4 \lambda}{\pi^{2}} \sum_{n=1}^{\infty} \frac{1}{(2 n-1)^{2}}\left\{e^{-G} n / 2\left[2 \ln \frac{1}{\varepsilon}+\right.\right.$

$\left.\sum_{m=1}^{\infty}\left(\frac{G_{n}}{2}\right)^{m} \frac{1-\varepsilon^{2 m}}{m ! m}\right]+\left[-2 \ln \frac{1}{\varepsilon}+\sum_{m=1}^{\infty}\left(\frac{G^{n}}{2}\right)^{m} \frac{\varepsilon^{2 m}}{m ! m} j \int_{\varepsilon}^{1} n^{2} F(n) e^{-G_{n} n^{2} / 2} d n\right.$ 
$\left.+\int_{\varepsilon}^{b}\left[2 \ln \frac{I}{r}-\sum_{m=1}^{\infty}\left(\frac{G}{2}\right)^{m} \frac{r^{2 m}}{m ! m}\right] r F(r) e^{-G} n r^{2 / 2} d r\right] \quad$ III-27

Equation III-12 defining the function $F(r)$ may be written as

$$
\begin{aligned}
F(r) & =\frac{1}{r}\left[\frac{8}{\pi^{2}} \sum_{n=1}^{\infty} \frac{1}{(2 n-1)^{2}} e^{-G n \cdot \frac{\left(1-r^{2}\right)}{2}}-\alpha\right] \\
& \frac{-R m N}{r} \int_{1}^{r} n^{2} F(n) \frac{8}{\pi^{2}} \sum_{n=1}^{\infty} \frac{1}{(2 n-1)^{2}} e^{-G \frac{\left(n^{2}-r^{2}\right)}{2}} d n
\end{aligned}
$$

III-28

where $\alpha=\frac{\int_{\varepsilon}^{l} \int_{0}^{1} v / \lambda d z d r}{2 b \pi \sigma R+\ln \frac{1}{\varepsilon}}$

Equation III-28 is a Volterra equation of the second kind The solution is iterative. The zeroth approximation is merely the non-homogeneous term; the first approximation is that term plus the integral of the zeroth approximation multiplied by the kernel; etc. The complete solution is

$$
F(r)=\left[1+\sum_{m=1}^{\infty} k_{f}^{m}\right]\left[\frac{8}{\pi^{2}} \frac{1}{r} \sum_{n=1}^{\infty} \frac{1}{(2 n-1)^{2}} e^{-G_{n} \frac{\left(1-r^{2}\right)}{2}}-\frac{\alpha}{r}\right] \quad \text { III-29 }
$$

where

$K_{F}[x(r)] \equiv \frac{8}{\pi^{2}} R+\int_{r}^{1} \frac{n^{2}}{r} x(n) \sum_{n=1}^{\infty} \frac{1}{(2 n-I)^{2}} e^{-G_{n} \frac{n^{2}-r^{2}}{2}}$ an III-30

The proof that this is a convergent solution is given in Append1X I。 The set of equations III-25,27,29,30 and II-73 form the solution of equation III-ll for the tangential velocity. In 
section III-4 below, these equations will be simplified to a much more practical form.

3. Solution of the Linearized Radial Momentum Equation

Since the tangential velocity solution is now known, equation III-10 may be solved for the radial velocity $u$ in terms of the function $F(r)$ and the pressure gradient $E d p / d r$. The pressure gradient is still an unknown; it is to be determined from the integral form of the conservation of mass flow equation II-80 after the radial velocity is found.

Equation III-10 will be solved by finite sine transform as equation III-II was. Again, there is symmetry about the mid plane $z=1 / 2$ so that only the odd terms in $n$ are retalned:

$$
\begin{array}{ll}
\bar{u}_{2 n-1}(r, n)=\pi \int_{0}^{1} u(r, z) \sin (2 n-1) \pi z d z & \text { III-3I } \\
u(r, z)=\frac{2}{\pi} \sum_{n=1}^{\infty} \bar{u}_{2 n-1}(r, n) \sin (2 n-1) \pi z d z & \text { III-32 }
\end{array}
$$

The transform of the second axial dirivative of the radial velocity is

$$
\frac{\partial^{2} \bar{u}_{2 n-1}}{\partial z^{2}}=-\pi^{2}(2 n-1)^{2} \bar{u}_{2 n-1}
$$

since $u(r, 0)=u(r, l)=0$. The transform of the tangential velocity $\mathrm{v}$ is given by equation III-24. The transform of the radial momentum equation III-10 is 
$\frac{1}{r} \frac{d \bar{u}_{2 n-1}}{d r}=G_{n} \bar{u}_{2 n-1}+\frac{2}{2 n-1} E \frac{d p}{d r}-\frac{2}{2 n-1} \frac{\lambda^{2}}{r^{3}}\left(1-\operatorname{RnN\alpha } \frac{\left(1-r^{2}\right)}{2}\right)$

$\left[e^{-G \frac{\left(1-r^{2}\right)}{2}}+R m N \int_{r}^{1} n^{2} F(n) e^{-G n \frac{n^{2}-r^{2}}{2}} d n\right]$

III-34

where $\quad G_{n}=\frac{\pi^{2} s^{2}}{\operatorname{Re}}(2 n-1)^{2}+R m N$

The solution of the linear, non-homogeneous, ordinary differential equation III-34, found by variation of parameters, is $\bar{u}_{2 n-1}(r, n)=e^{-G \frac{\left(1-r^{2}\right)}{2}}\left[d_{n}+\frac{2 \lambda^{2}}{2 n-1}\left(\frac{1}{r}-1-\frac{R m N a}{2}\left(r+\frac{1}{r}\right)+R m N a\right)\right]$ $+\frac{21^{2}}{2 n-1} \operatorname{RmN}\left[\frac{\mathrm{RmNa}}{2}\left(r+\frac{1}{r}\right)-\frac{1}{r}\right] \int_{1}^{r} n^{2} F(n) e^{-G \frac{\left(n^{2}-r^{2}\right)}{2}} d n$ $-2 \frac{\lambda^{2}}{2 n-1} \operatorname{RmN} \int_{1}^{r}\left[\frac{R m N \alpha}{2}\left(n+\frac{1}{n}\right)-\frac{1}{n}\right] n^{2} F e^{-G} \frac{\left(n^{2}-r^{2}\right)}{2} d n$ $+\frac{2}{2 n-1} \int_{1}^{r} n E \frac{d p}{d n} e^{-G \frac{n^{2}-r^{2}}{2}} d n$

The transform of the radial boundary condition II-32-a is At $r=1 ; \quad \bar{u}_{2 n-1}=-\frac{2}{2 n-1}$ III -36

This boundary condition allows the integration constant $d_{n}$ to be evaluated and yields

$\bar{u}_{2 n-1}(r, n)=\frac{2}{2 n-1} e^{-G \frac{\left(1-r^{2}\right)}{2}}\left[\lambda^{2}\left(\frac{1}{r}-1-\frac{R m N a}{2}\left(r+\frac{1}{r}\right)+R m N \alpha\right)-1\right]$
$+\frac{2 \lambda^{2}}{2 n-1} R m N \int_{r}^{1}\left[\frac{1}{r}-\frac{1}{n}+\frac{R m N \alpha}{2}\left(r+\frac{1}{n}-r-\frac{1}{r}\right)\right] n^{2} F(n) e^{-G \frac{\left(n^{2}-r^{2}\right)}{2}} d n$
$-\frac{2}{2 n-1} \int_{r}^{1} n E \frac{d p}{d n} e^{-G \frac{n^{2}-r^{2}}{2}} d n$ 
The inverse transform of equation III-37 is

$$
\begin{aligned}
& u(r, z)=\frac{4}{\pi} \sum_{n=1}^{\infty} \frac{\sin (2 n-1) \pi z}{2 n-1}\left\{e^{-G n \frac{\left(1-r^{2}\right)}{2}}+\lambda^{2} e^{-G n \frac{\left(1-r^{2}\right)}{2}}\right. \\
& {\left[\frac{1}{r}-1+\frac{R m N \alpha}{2}\left(2-r-\frac{J}{r}\right)\right]+\lambda^{2} R m N \int_{r}^{1}\left[\frac{1}{r}-\frac{1}{n}+\frac{R m N \alpha}{2}\left(n-r+\frac{1}{n}-\frac{1}{r}\right)\right]} \\
& n^{2} F(n) e^{-G \frac{\left(n^{2}-r^{2}\right)}{2}} d n-\int_{r}^{1} n E \frac{d p}{d n} e^{-G \frac{n^{2}-r^{2}}{2}} d n
\end{aligned}
$$

Equation III-38 gives the radial velocity $u$ as a function of the pressure gradient $\mathrm{E} d \mathrm{p} / \mathrm{dr}$. Now the integral form of the conservation of mass equation II- 80 may be used to determine the pressure gradient:

$$
\int_{0}^{1} u d z=-1 / r
$$

Subst1tution of equation III-38 into II-80 yields

$$
-\int_{r}^{1} n E \frac{d p}{d n} \frac{8}{\pi^{2}} \sum_{n=1}^{\infty} \frac{1}{(2 n-1)^{2}} e^{-G_{n} \frac{\left(n^{2}-r^{2}\right)}{2}} d n+\frac{1}{r}
$$

$+\lambda^{2} \mathrm{RmN} \int_{r}^{1}\left[\frac{1}{r}-\frac{1}{n}+\frac{\mathrm{RnN \alpha}}{2}\left(n-r+\frac{1}{n}+\frac{1}{r}\right)\right] n^{2} F(n) \frac{8}{\pi} \sum_{n=1}^{\infty} \frac{e^{-G_{n} \frac{\left(n^{2}-r^{2}\right)}{2}}}{(2 n-1)^{2}} d n$

$$
+\left[\lambda^{2}\left(\frac{1}{r}-1-\frac{P m N a}{2}\left(r+\frac{1}{r}\right)+R m N \alpha\right)-1\right] \frac{8}{\pi^{2}} \sum_{n=1}^{\infty} \frac{1}{(2 n-1)^{2}} e^{-G_{n} \frac{\left(1-r^{2}\right)}{2}}=0
$$

$$
\text { III-39 }
$$

Equation III-39 is a Volterra equation of the first kind. The integral involving $F(r)$ is a known function from equation III-29. Equation III-39 may be transformed into a Volterra equation of the second kind merely by differentiation: 


$$
\begin{aligned}
& E \frac{d p}{d r}=\left\{\frac{1}{r^{3}}-\frac{8}{\pi^{2}} \sum_{n=1}^{\infty} \frac{1}{(2 n-1)^{2}} e^{-G_{n} \frac{\left(1-r^{2}\right)}{2}}\left[-\lambda^{2}\left(\frac{1}{r^{3}}+\frac{R m N \alpha}{2}\left(\frac{1}{r}-\frac{1}{r^{3}}\right)\right)\right.\right. \\
& \left.-G_{n}+\lambda^{2} G_{n}\left(\frac{1}{r}-1-\frac{\mathrm{RnNa}^{2}}{2}\left(r+\frac{1}{r}\right)+\mathrm{RnN \alpha}\right)\right] \\
& +\lambda^{2} \mathrm{RmN} \int_{r}^{1}\left[\frac{\mathrm{RmN \alpha}}{2 r}+\frac{n^{2}}{r^{3}}\left(1-\frac{\mathrm{RmN \alpha}}{2}\right)\right] F(n) \frac{8}{\pi^{2}} \sum_{n=1}^{\infty} \frac{e^{-G_{n} \frac{\left(n^{2}-r^{2}\right)}{2}}}{(2 n-1)^{2}} d n \\
& \left.-\lambda^{2} \mathrm{RNN} \int_{r}^{1}\left[\frac{1}{r}-\frac{1}{n}+\frac{R \mathrm{mNa}}{2}\left(n-r+\frac{1}{n}-\frac{1}{r}\right)\right] n^{2} F(n) \frac{8}{\pi} \sum_{n=1}^{\infty} \frac{G_{n} e^{-G_{n} \frac{\left(n^{2}-r^{2}\right)}{2}}}{(2 n-1)^{2}} d n\right\} \\
& +\int_{r}^{1} n E \frac{d p}{d n} \frac{8}{\pi^{2}} \sum_{n=1}^{\infty} \frac{1}{(2 n-1)^{2}} e^{-G \frac{\left(n^{2}-r^{2}\right)}{2}} d \eta \quad \text { III-40 }
\end{aligned}
$$

This Volterra equation of the second kind may be solved in the same manner that equation III-28 was:

$$
\begin{aligned}
& \mathrm{E} \frac{d \mathrm{p}}{d r}=\left[1+\sum_{m=1}^{\infty} \mathrm{K}_{\mathrm{p}}^{m_{3}}\right] \frac{1}{r^{3}}+\frac{8}{\pi^{2}} \sum_{n=1}^{\infty} \frac{1}{(2 n-1)^{2}} e^{-G_{n} \frac{\left(1-r^{2}\right)}{2}}\left[G_{n}+\frac{\lambda^{2}}{r^{3}}\right. \\
& \left.\left(1-R m N a \frac{\left(1-r^{2}\right)}{2}\right)+\lambda^{2} G_{n}\left(1-\frac{1}{r}+\frac{R n N a}{2}\left(r+\frac{1}{r}\right)-R n N a\right)\right]+\frac{\lambda^{2} R m N}{r^{3}} \\
& \int_{r}^{1}\left[1-\frac{R m N a}{2}+\frac{r^{2}}{n^{2}} \frac{R m N a}{2}\right] n^{2} F(n) \frac{8}{\pi^{2}} \sum_{n=1}^{\infty} \frac{e^{-G \frac{\left(n^{2}-r^{2}\right)}{2}}}{(2 n-1)^{2}} d n-\lambda^{2} R m N \int_{r}^{1} \\
& \left.\left[\frac{1}{r}-\frac{1}{n}+\frac{P m N \alpha}{2}\left(n-r+\frac{1}{n}-\frac{1}{r}\right)\right] n^{2} F(n) \frac{8}{\pi^{2}} \sum_{n=1}^{\infty} \frac{G_{n} e^{-G_{n} \frac{\left(n^{2}-r^{2}\right)}{2}}}{(2 n-1)^{2}} d n\right\} \\
& \text { III-4I }
\end{aligned}
$$

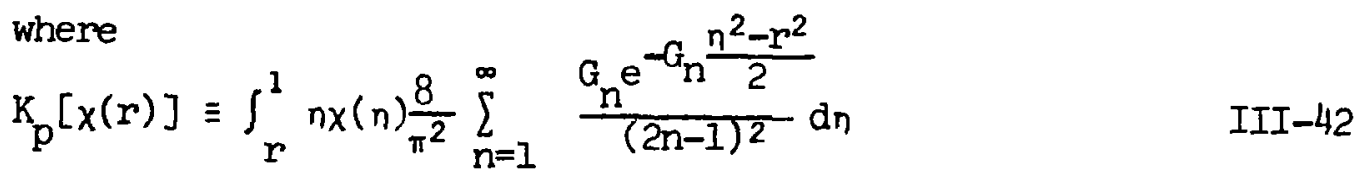

54 
The proof that this solution for the radial pressure gradient is convergent is given in Appendix I.

With the radial velocity $u$ known, it is a simple matter to solve the continuity equation II-75 for the axial velocity w:

$w=\frac{4}{\pi^{2}} \sum_{n=1}^{\infty} \frac{\cos (2 n-1) \pi z}{(2 n-1)^{2}}\left\{e^{-G_{n} \frac{\left(1-r^{2}\right)}{2}}\left[\lambda^{2} G_{n}-\left(1+\lambda^{2}\right)\left(\frac{1}{r}+G_{n} r\right)\right.\right.$

$\left.+\lambda^{2} \mathrm{RmNa}\left(\frac{1}{r}-1-\frac{G_{n}}{2}(1-r)^{2}\right)\right]+r E \frac{d p}{d r}+\lambda^{2} \mathrm{RmN} \int_{r}^{1}\left[G_{n}(n-r)\left(1-\mathrm{RnNa} \frac{(1-n r)}{2}\right)\right.$

$\left.-\frac{P m N \alpha}{2 r}\left(n r-I-n^{2}\right)-\frac{1}{r}\right] \quad n F(n) e^{-G_{n} \frac{\left(n^{2}-r^{2}\right)}{2}} d n-\left(G_{n} r+\frac{1}{r}\right)$

$\left.\int_{r}^{1} n E \frac{d p}{d n} e^{-G_{n} \frac{\left(n^{2}-r^{2}\right)}{2}} d n\right\}$

III-43

Thus solution for the axial velocity is not able to satisfy the boundary conditions at $r=1(w(1, z)=0)$ since the differential equations do not contain radial derivatives of the axial velocity. This is a shortcoming cormon to boundary layer theory analyses. The solution for the axial velocity $w$ does satisfy the no flow boundary conditions at the plates $z=0$ and $I$ by virtue of the fact that $\sum_{n=1}^{\infty} \frac{1}{(2 n-1)^{2}}\left\{r E \frac{d p}{d r}-\left(G_{n} r+\frac{1}{r}\right) \int_{r}^{1} n E \frac{d p}{d n} e^{-G_{n} \frac{\left(n^{2}-r^{2}\right)}{2}} d n+e^{-G_{n} \frac{\left(1-r^{2}\right)}{2}}\right.$ $\left[G_{n} \lambda^{2}-\left(1+\lambda^{2}\right)\left(G_{n} r+\frac{1}{r}\right)+\lambda^{2} R m N a\left[\frac{1}{r}-1-G_{n} \frac{(1-r)^{2}}{2}\right\}\right]+\lambda^{2} R m N \int_{r}^{1}$ $\left.\left[G_{n}(n-r)\left(1-\mathrm{PmN \alpha} \frac{(1-\eta r)}{2}\right)-\frac{\mathrm{RmN \alpha}}{2 r}\left(n r-1-n^{2}\right)-\frac{1}{r}\right] n F(n) e^{-G_{n} \frac{\left(n^{2}-r^{2}\right)}{2}} \mathrm{~d} n\right\}=0$ III-44 
This equality may be verifled by a combination of $\left(\pi^{2} r / 8\right)$ times equation III-40 and $(I / r)$ times equation III-43.

\section{A Further Simplification}

Equations III-25, 39 and 43 for the velocity components $u, v$, and $w$ are valid in general; there are no limitations on the paramet ters $\lambda, s^{2} /$ Re and RmN. As these solutions stand, they are too complex for practical use, even for numerical computations. In this section, these equations will be simplified to a more practical form by making use of a certatn approximation explained below.

The function which makes it impossible to transform the integral equations III-28 and 39 into differential form is

$$
f(x) \equiv \frac{8}{\pi^{2}} \sum_{n=1}^{\infty} \frac{1}{(2 n-1)^{2}} e^{-G_{n} x}
$$

If this function were approximated it would enable $F(r)$ and $\frac{d p}{d r}$ to be found in closed form and thus greatly simplify the solutions. Fortunately, the serles is highly convergent and may be roughly approximated by its first term; therefore assume

$$
f(x)=e^{-G_{1} x}=e^{-(\theta+R m N) x}
$$

where

$$
\theta \equiv \pi^{2} \mathrm{~s}^{2} / \mathrm{Re}
$$

The error introduced by this approximation is fully analyzed in Appendix II. There is no error if Reynolds number is infinity 
while as the Reynolds number approaches zero, the error asymptotIcally approaches $23 \%$.

Now equation III-28 may be replaced by

$$
r F(r)=e^{-(\theta+F m N) \frac{l-r^{2}}{2}}-\alpha-F m N \int_{1}^{r} \eta^{2} F(\eta) e^{-(\theta+R m N) \frac{\eta^{2}-r^{2}}{2}} d \eta
$$

Whereas it was impossible to express equation III-28 in a differential form because of the infinite sum, equation III-48 is equivalent to the differential equation

$$
(r F)^{\prime}=r[\theta(r F)+(\mathrm{RmN}+\theta) \alpha]
$$

The solution of equation III- 49 is

$$
r F(r)=e^{-\theta \frac{\left(1-r^{2}\right)}{2}}-\alpha-R m a \frac{1-e^{-\theta \frac{\left(1-r^{2}\right)}{2}}}{\theta} \quad \text { III-50 }
$$

where the constant of integration has been determined by substitutIng the form

$$
r F=-\alpha\left(1+\frac{R m N}{\theta}\right)+c e^{-\theta \frac{1-r^{2}}{2}}
$$

into equation III-48.

This closed form solution for the function $F(r)$ greatly simpI1fles the solution for the tangential velocity v; equation III-25 is now 
$v(r, z)=\frac{4}{\pi} \frac{\lambda}{r} \sum_{n=1}^{\infty} \frac{\sin (2 n-1) \pi z}{(2 n-1)}\left[\frac{F m N}{a_{n}-\theta}\left(e^{-\theta^{\left(\frac{\left.1-r^{2}\right)}{2}\right.}}-\pi m N \alpha \frac{1-e^{-\theta \frac{\left(1-r^{2}\right)}{2}}}{\theta}\right)\right.$

$\left.\left.+\left(1-\frac{R m N}{G_{n}-\theta}\right)\left(1+\frac{R m N \alpha}{G_{n}}\right) e^{-G_{n} \frac{\left(1-r^{2}\right)}{2}}-\frac{B m N \alpha}{G_{n}}\right)\right]$

where $G_{n}=\frac{\pi^{2} s^{2}}{R e}(e n-1)^{2}+R m N$ and $\theta=\pi^{2} s^{2} /$ Re

Equation III-5l, giving the solution of the tangential velocity $v$, is plotted for various values of the parameters in figures 2 and 3. These figures will be discussed in chapter $V$.

Equation III-5I expresses the tangential velocity $v$ in terms of the external current parameter $\alpha$. It is now possible to obtain an explicit expression for $\alpha$ by using the difinition II-73 and equation III-51. Carrying out the indicated integration yields the result

$$
\begin{aligned}
& \sum_{n=1}^{\infty} \frac{1}{(2 n-1) 2}\left\{\frac{R m N}{G_{n}-\theta} e^{-\theta / 2}\left[\ln \frac{1}{\varepsilon}+\frac{1}{2} \sum_{m=1}^{\infty} \frac{\theta^{m}\left(1-\varepsilon^{2 m}\right)}{2^{m} m ! m}\right]\right. \\
& \alpha=\frac{\left.+\left(1-\frac{R m N}{G_{n}^{-\theta}}\right) e^{-\theta / 2}\left[\operatorname{In} \frac{1}{\varepsilon}+\frac{1}{2} \sum_{m-1}^{\infty} \frac{G_{n}^{m}\left(1-\varepsilon^{2^{m}}\right)}{2^{m} m ! m}\right]\right\}}{\frac{\pi^{2}}{8}\left(2 \pi \sigma \tilde{R} b+\ln \frac{1}{\varepsilon}\right)+\sum_{n=1}^{\infty} \frac{1}{(2 n-1)^{2}} \cdot\left(-\frac{R m N}{G_{n}}\left(1-\frac{R_{m N}}{G_{n}-\theta}\right) e^{-G_{n} / 2}\right.} \text { III-52 } \\
& {\left[\ln \frac{1}{\varepsilon}+\frac{1}{2} \sum_{m=1}^{\infty} \frac{G_{n}^{m}\left(1-\varepsilon^{2^{m}}\right)}{2^{m} m ! m}\right]+\frac{F_{m N}}{G_{n}}\left(1-\frac{R^{m N}}{G_{n}-\theta}\right) \ln \frac{1}{\varepsilon}} \\
& \left.+\frac{(R m N)^{2}}{G_{n}{ }^{-\theta}} \frac{1-e^{-\theta / 2}}{\theta} \ln \frac{1}{\varepsilon}+\frac{(R m N)^{2}}{G_{n}^{-\theta}} \frac{1}{2} e^{-\theta / 2} \sum_{m=1}^{\infty} \frac{\theta^{m-1}\left(1-\varepsilon^{2 m}\right)}{2^{m} m ! m}\right\}
\end{aligned}
$$


This expression for the current $\alpha$ may be greatly simplified if use is made of equation III-12 defining $F(r)$ and if $\alpha$ is approximated to the same accuracy as $F(r)$ is. This yields the simplified result

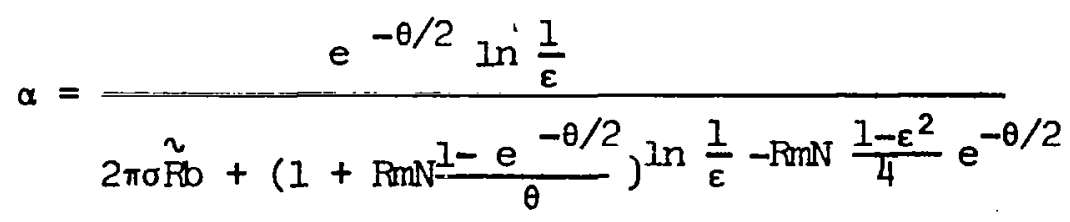

III -53

This parameter $\alpha$ is a measure of the electric current flowing in the external circuit. Thus if the external resistance $\tilde{R}$ becomes infinite, $\alpha$ becomes zero. If the external resistance becomes zero a. reaches a finite maximum limited by the internal resistance of the device. The parameter $\alpha$ does not go to zero as the magnetic term $\mathrm{RmN}$ becomes small. This is not the fault of the analysis but is due to the nature of the nondimensionalization process. As the magnetic influence becomes small, the true (dimensional) current does become small also. Note that in equation III-5I for the tangential velocity the parameter $\alpha$ is always multiplied by the magnetic 
parameter $\mathrm{RmN}$.

The set of equations III-5I and 53 now form the solution to tangential momentum equation III-II; the solution for the tangential velocity $v$ is now known. This solution is applicable for all values of Reynolds number and magnetic parameter but is more accurate for large Reynolds numbers than for small (see Appendix II).

In a similar manner, the solution for the radial velocity III-38 may be much simplified. Using the same approximation (equations III-45 and 46) that was employed in equation III-28, equation III-39 for the pressure gradient is reduced to

$-\int_{r}^{l} n E \frac{d p}{d n} e^{-(\theta+R m N) \frac{n^{2}-r^{2}}{2}} d \eta+e^{-(\theta+R m N) \frac{1-r^{2}}{2}}\left[-1+\lambda^{2}\left(\frac{1}{r}-1+\frac{R m N a}{2}\right.\right.$

$\left.\left.\left(2-r-\frac{1}{r}\right)\right)\right]+\lambda^{2} \int_{r}^{1}\left[\frac{n^{2}}{r}\left(1+\mathrm{RmNa} \frac{1+r^{2}}{2}\right)-n\left(1-\mathrm{RmNa} \frac{1+n^{2}}{2}\right)\right]$

$\operatorname{RmNF}(n) e^{-(\theta+\operatorname{RmN}) \frac{n^{2}-r^{2}}{2}} \mathrm{~d} n+\frac{1}{r}=0$

This equation may be directly differentiated and solved for the pressure gradient:

$E \frac{d p}{d r}=\frac{1}{r^{3}}+\frac{\mathrm{RmN}+\theta}{r}+\frac{\lambda^{2}}{r^{3}} e^{-(\theta+\mathrm{RmN}) \frac{1-r^{2}}{2}}\left[1-\mathrm{RmNa} \frac{1-r^{2}}{2}\right]$

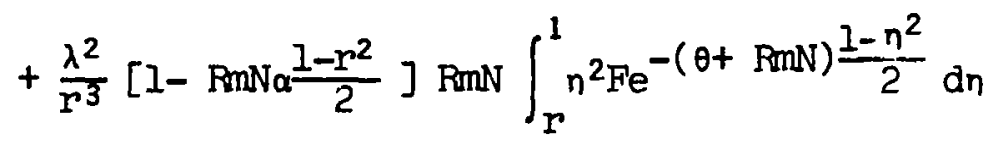


or, employing equation III-50 to eliminate the integral,

$E \frac{d p}{d r}=\frac{1}{r^{3}}+\frac{\theta+P m N}{r}+\frac{\lambda^{2}}{r^{3}}\left[1-P M N \alpha \frac{1-r^{2}}{2}\right]\left[e^{-\theta \frac{1-r^{2}}{2}}-\mathrm{RmN \alpha} \frac{1-e^{-\theta \frac{1-r^{2}}{2}}}{\theta}\right]$

III-56

Equation III-56 may be easily verified as correct for the case where Reynolds number becomes very large. In this case $\theta \longrightarrow 0$ and

$E \frac{d p}{d r}=\frac{1}{r^{3}}+\frac{R m N}{r}+\frac{\lambda^{2}}{r^{3}}\left[1-\mathrm{RmNa} \frac{1-r^{2}}{2}\right]^{2}$

For Inviscid flow, $u=-1 / r$ and $v=\lambda / r\left(1-\mathrm{RmN} \alpha \frac{1-r^{2}}{2}\right)$. The nonlinear radial momentum equation II-76 may be solved for $E \frac{d p}{d r}$ for this special case; the result is identical to that above. This is a check on the validity of the anaiysis to this point.

Substituting equations III-50 and 56 into equation III-38 yields the simplified expression for the radial velocity: 


$$
\begin{aligned}
& u=\frac{4}{\pi} \sum_{n=1}^{\infty} \frac{\sin (2 n-1) \pi z}{2 n-1}\left\{-\frac{1}{r}+\lambda^{2}\left(1-\frac{\pi m N}{G_{n}-\theta}\right)\left(1-\frac{R m N \alpha}{2}\right)\left[\frac{e^{-G_{n} \frac{\left(1-r^{2}\right)}{2}}-e^{-\theta\left(1-r^{2}\right)}}{r}\right.\right. \\
& \left.+\operatorname{PmN\alpha } \frac{1}{r} \frac{1-e^{-\theta \frac{\left(1-r^{2}\right)}{2}}}{\theta}-\frac{\mathrm{R}_{\mathrm{nNa}}}{G_{n}} \frac{1-e^{-G_{n} \frac{\left(1-r^{2}\right)}{2}}}{r}\right] \\
& +\lambda^{2}\left(1-\frac{R m N}{G_{n}-\theta}\right)\left(1+\frac{R n N \alpha}{G_{n}}\right) \frac{R m N \alpha}{2}(1-r) e^{-G_{n} \frac{\left(1-r^{2}\right)}{2}} \\
& -\left(1-\frac{R m N}{G_{n}-\theta}\right)\left(G_{n}-\theta-\lambda^{2} \frac{(R m N a)^{2}}{2 G_{n}} \int_{r}^{1} e^{-G_{n} \frac{\left(n^{2}-r^{2}\right)}{2}} d n\right. \\
& +\lambda^{2}\left(1-\frac{P m N}{G_{n}-\theta}\right)\left[\left(G_{n}-\theta\right)\left(1-\frac{P m N \alpha}{2}\right)-\frac{R m N \alpha}{2}\right] \int_{r}^{1}\left[e^{-\theta \frac{\left(1-n^{2}\right)}{2}}-R m N \alpha \frac{1-e^{-\theta \frac{\left(1-n^{2}\right)}{2}}}{\theta}\right] \\
& e^{-G_{n} \frac{\left(n^{2}-r^{2}\right)}{d n t}}
\end{aligned}
$$

The solution for the radial veloc1ty $u$ given by equation III57 is plotted in figures 4,5 and 6 for various values of the governIng parameters. The results will be discussed in chapter V. The integrals appearing in equation III-57 may be expressed in terms of error functions.

The solution for the axial velocity $w$ is now, instead of equation III-43, 


$$
\begin{aligned}
& w=\frac{4}{\pi^{2}} \sum_{n=1}^{\infty} \frac{\cos (2 n-1) \pi z}{(2 n-1)^{2}}\left[1-\frac{R m N}{G_{n}-\theta}\right]\left\{G_{n}-\theta+\lambda^{2}[R m N \alpha / 2\right. \\
& \left.-G_{n}\left(\frac{1-P n N a}{2}\right)\right]\left[e^{-G_{n} \frac{\left(1-r^{2}\right)}{2}}-e^{-\theta \frac{\left(1-r^{2}\right)}{2}}+R m N a \frac{1-e^{-\theta \frac{\left(1-r^{2}\right)}{2}}}{\theta}\right. \\
& \left.-\frac{R m N \alpha}{G_{n}}\left(1-e^{-G_{n} \frac{\left(1-r^{2}\right)}{2}}\right)\right]-\lambda^{2} \frac{R m N a}{2}\left(1+\frac{R m N \alpha}{G_{n}}\right)\left(\frac{1}{r}+G_{n} r\right)(1-r) e^{-G_{n}^{\prime}} \frac{\left(1-r^{2}\right)}{2} \\
& -\left(\frac{1}{r}+G_{n} r\right)\left[G_{n}-\theta-\lambda^{2} \frac{(R m N a)^{2}}{2 G_{n}}\right] \int_{r}^{1} e^{-G_{n} \frac{\left(n^{2}-r^{2}\right)}{2}} d n \\
& -\lambda^{2}\left(\frac{1}{r}+G_{n} r\right)\left[\left(G_{n}-\theta\right)\left(1-\frac{R m N a}{2}\right)-\frac{P m N a}{2}\right] \int_{r}^{l} \\
& {\left[e^{-\theta \frac{\left(1-n^{2}\right)}{2}}-\operatorname{RnN\alpha } \frac{1-e^{-\theta \frac{\left(1-n^{2}\right)}{2}}}{\theta}\right] e^{-G_{n} \frac{n^{2}-r^{2}}{2}} d n}
\end{aligned}
$$


CHAPTER IV

\section{CALCULATION OF THERMAL AND EIECTROMAGNETIC \\ VARIABLES}

In this chapter, the solution of the zeroth order set of unknown will be completed. This will involve the determination of $T_{0}, J_{r 0}, J_{\phi_{0}}, E_{r_{0}}$ and $B_{\phi 0^{*}}$. In addition, such quantities as heat conduction at the walls, and extermal electric fleld will be calculated. In the following, the subscript zero will be omitted for convenience; it will be understood that the variables are part of the zeroth order set of unknowrs.

\section{Solution of the Energy Equation for Prescribed End Wall Temperature.}

Two of the basic assumptions made in chapter I concerning the nature of the working fluid are that its density and electric conductivity are constant. If the fluid is actually compressible, the validity of the assumption of constant density depends upon the Mach number and also upon the temperature distribution in the fluid. Also, if the working fluid is a hot gas whose conductivity is based upon thermal ionization, the conductivity may be a strong function of temperature. In this case the cooling effect of the end walls may quench the fluid near the walls and thereby decrease its elec- 
tric conductivity. For these reasons, it is of interest to calculate the temperature variation in the region of interest and the heat transfer through the end walls.

The energy equation was developed in chapter II and is

$u \frac{\partial T}{\partial r}+w \frac{\partial T}{\partial z}=\frac{s^{2}}{\operatorname{RePr}} \frac{\partial^{2} T}{\partial z^{2}}+E c \frac{s^{2}}{\operatorname{Re}}\left[\left(\frac{\partial v}{\partial z}\right)^{2}+\left(\frac{\partial u}{\partial z}\right)^{2}\right]$

$+\operatorname{RmN~Ec}\left[u^{2}+\left(\frac{\lambda \alpha}{\mathrm{r}}+v-\int_{0}^{1} v d z\right)^{2}\right]$

where the functions $u, v$, and $w$ are known and are given by equations III-57, 51 and 58 respectively.

The boundary conditions which the solution must satisfy are

At $\mathrm{z}=0$ and $\mathrm{I}$

$$
T=T W / T_{R}
$$

II-30-d-11

At $r=1$

$T=1$

II-32-d

Equation II-78 is considerably simplifled from 1ts inftial form in equation II-9. However, as 1 t stands at present, a tractable solution is not possible. Some reasonable simplification must be found.

Let the analysis now be restricted to the range of parameters which is of interest for power generation. That is, consider

$$
\mathrm{s}^{2} / \operatorname{Re} \ll \operatorname{RmN} \quad \text { IV-1 }
$$

The flow regime is one of magnetohydrodynamic boundary layer flow. This assumption allows a considerable simplification in the nonhomogeneous terms in equation II-78. 
With this approximation it is seen that the foule heating terms, characterized by RnNEc, will be much larger than the viscous heating terms, characterized by $\mathrm{Ec} \mathrm{s}^{2} / \mathrm{Re}$, except possibly in the boundary layers. Therefore, the viscous heating terms will be neglected.

Now consider the joule heating terms. With the analysis restricted to the boundary layer type of flow, the foule heating terms may be closely approximated by their inviscid values. That is, instead of using equations III-57 and 51 to represent the velocities $u$ and $v$, equations $A-18$ and 19 from Appendix III will be used. The error introduced by this approximation tends to cancel the error introduced by the neglect of the viscous heating terms.

With the same reasoning which accompanied the Iinearization of the momentum equations in section III-1, the non-linear convection terms of the energy equation will be linearized as follows:

$$
\left(u \frac{\partial}{\partial r}+w \frac{\partial}{\partial z}\right) \longrightarrow\left(-\frac{1}{r} \frac{\partial}{\partial r}\right) \quad I V-2
$$

As a result of these simplifications the energy equation II78 may now be written

$$
-\frac{1}{r} \frac{\partial T}{\partial r}=\frac{\mathrm{s}^{2}}{\operatorname{RePr}} \frac{\partial^{2} \mathrm{~T}}{\partial \mathrm{z}^{2}}+\mathrm{RmNEc} \frac{1+\lambda^{2}}{\mathrm{r}^{2}} \underline{\alpha}^{2}
$$

Using the finite sine transform, the solution of equation IV -3 is 
$T=\frac{T_{W}}{T_{R}}+\frac{4}{\pi} \sum_{n=1}^{\infty} \frac{\sin (2 n-1) \pi z}{(2 n-1)}\left\{\left(1-\frac{T_{W}}{T_{R}}\right) e^{-\frac{\pi^{2} s^{2}}{\operatorname{RePr}}(2 n-1)^{2} \frac{1-r^{2}}{2}}\right.$

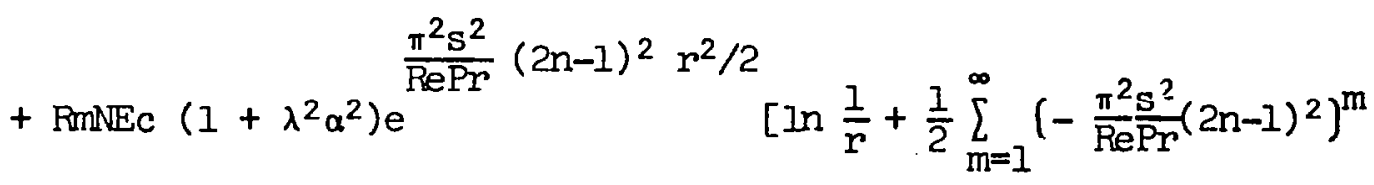

$\left.\frac{1-r^{2 m}}{m ! m}\right]$

IV-4

This solution is valid for small inverse Reynolds number, $\mathrm{s}^{2} / \mathrm{Re}$, compared with the magnetic parameter, RmN. This solution ignores the effect of the velocity boundary layers on the temperature profile but includes the effect of the end walls in that the thermal boundary condition II-30-d-ii is satisfied. This solution for the temperature is plotted in figures 7 and 8 for $r=.7$ and for various values of the governing parameters, $\operatorname{RmNEc}\left(1+\lambda^{2} \alpha^{2}\right)$ and $\operatorname{RePr} / \mathrm{s}^{2}$. The results are discussed in chapter $\mathrm{V}$.

The heat transfer at the wall is given by the non dimensional heat conduction equation

$$
q=\frac{b}{k T_{R}} q^{*}=-\frac{\partial T}{\partial z}
$$

Employing the known solution for the temperature, equation IV-4, yields the heat conduction at the wall.

$$
\begin{aligned}
& q=-\frac{4}{\pi^{2}} \sum_{n=1}^{\infty} \frac{1}{2 n-1^{2}}\left\{\left(1-\frac{T_{w}}{T_{R}}\right) e^{-\frac{\pi^{2} s^{2}}{\operatorname{RePr}}(2 n-1)^{2} \frac{1-r^{2}}{2}}\right. \\
& +\operatorname{RmNEc}\left(1+\lambda^{2} \alpha^{2}\right) e^{\frac{\pi^{2} s^{2}}{\operatorname{RePr}(2 n-1)^{2} r^{2} / 2}}\left[\ln \frac{1}{r}+\frac{1}{2} \sum_{m=1}^{\infty}\left(-\frac{\pi^{2} s^{2}}{\operatorname{RePr}} \frac{(2 n-1)^{2}}{2}\right)^{m}\right. \\
& \left.\left.\frac{1-r^{2 m}}{m ! m}\right]\right\} \\
& \text { IV }-6
\end{aligned}
$$


This solution has the sane range of valldity as the temperature solution has; Reynolds number must be large. The solution for the heat transfer at the wall is given in table 4 for various valLes of the parameters $\operatorname{RePr} / \mathrm{s}^{2}$ and $\operatorname{RmNEc}\left(I+\lambda^{2} \alpha^{2}\right)$.

2. Solution of the Energy Equation for

Adiabatic End Walls

For the case of adiabatic end walls, the solution to the energy equation must satisfy

$$
\begin{array}{rlrl}
\text { At } z=0 \text { and } z=1 & \frac{\partial T}{\partial z}=0 & \text { II }-30-d-1 \\
\text { At } r=1 & T=1 & \text { II }-32-d
\end{array}
$$

Repeating the assumptions made in the section above leads again to an energy equation in the form of equation IV-3. The solution for this case is

$$
T=I+\operatorname{RnN} \operatorname{Ec}\left(1+\lambda^{2} \alpha^{2}\right) \operatorname{In} \frac{1}{r}
$$

This solution is valid for large Reynolds number; there is no restriction upon the range of the parameters $\mathrm{RmN}, \mathrm{Ec}$ and $\lambda$. This solution is independent of the axial coordinate; the temperature distribution is logarithmic in the radius. The strength of the variation depends upon the amount of joule heating in the region of interest. 
3. Calculation of Electromagnetic

Variables

For completeness, the remaining undetermined electromagnetic variables of the zeroth order set of unknowns $J_{r}, J_{\phi}, E_{r}$ and $B_{\phi}$ w11l now be calculated.

A. ELECTRICAL CURRENTS

The zeroth order radial current, the component which ylelds useful electric power, is given by equation II-74

$$
J_{r}=\frac{\lambda \alpha}{r}+v-\int_{0}^{1} v d z
$$

The tangential velocity $v$, and external current, $\alpha$ are known from equations III-5I and 53. They yleld the result:

$J_{r}=\frac{\lambda \alpha}{r}+\frac{4 \lambda}{\pi r} \sum_{n=1}^{\infty}\left[\frac{\sin (2 n-1) \pi z}{(2 n-1)}-\frac{2}{\pi} \frac{1}{(2 n-1)^{2}}\right]\left[\frac{R m N}{G_{n}-\theta}\left[e^{-\theta \frac{1-r^{2}}{2}}\right.\right.$ - PmNa $\left.\left.\frac{1-e^{-\theta \frac{1-r^{2}}{2}}}{\theta}\right]+\left(1-\frac{R m N}{G_{n}-\theta}\right)\left[1+\frac{R m N a}{G_{n}} e^{-G_{n} \frac{1-r^{2}}{2}}-\frac{R m N a}{G_{n}}\right]\right\}$

$$
\alpha=\frac{c^{-\theta / 2} \ln \frac{1}{\varepsilon}}{2 \pi \sigma \tilde{R} b+\left(1+R m N \frac{1-e^{-\theta / 2}}{\theta}\right) \ln \frac{1}{\varepsilon}-\operatorname{RmN} \frac{1-\varepsilon^{2}}{4} e^{1-\theta / 2}} \quad \text { III-5I }
$$

where $G_{n}=\theta(2 n-1)^{2}+R n N \quad$ and $\theta=\pi^{2} s^{2} / \operatorname{Re}$

The first term on the right hand side of equation IV-8 represents the electric current which passes through the external circuit. The expression ( $2 \pi \circ \mathrm{Rb})$ in the denominator of the solution 
for this current III-51 is the ratio of the resistance of the external circuit $\vec{R}$ to the intemal resistance of the flow region

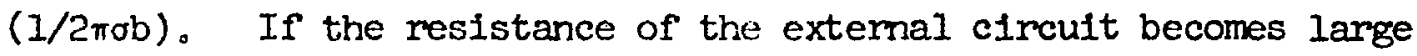
compared with the internal resistance, the external current becomes very small. If the external resistance is small compared with the internal resistance, the external current approaches a maximum limited by the internal resistance of the device.

The tangential current $J_{\phi}$ is equal to the negative of the radial velocity by equation II-77; the radial velocity is given by equation III-57. The zeroth order axial current is identically zero from equation II-59.

\section{B。 ELECTRIC FIFLDS}

The zeroth order radial electric field may be expressed in terms of velocities by a combination of equations II-53 and 73:

$$
E_{r}=\frac{\lambda \alpha}{r}-\int_{0}^{1} v d z
$$

Again the tangential velocity, $v$, is known from equation III-51; this yields

$E_{r}=\frac{\lambda \alpha}{r}-\frac{8}{\pi^{2}} \frac{\lambda}{r} \sum_{n=1}^{\infty} \frac{1}{(2 n-1)^{2}}+\frac{R m N}{G_{n}-\theta}\left[e^{-\theta \frac{1-r^{2}}{2}}-R m N \alpha \frac{1-e^{-\theta \frac{1-r^{2}}{2}}}{\theta}\right]$

$\left.+\left(1-\frac{R m N}{G_{n}-\theta}\right)\left[\left(1+\frac{P m N \alpha}{G_{n}}\right) e^{-G_{n} \frac{1-r^{2}}{2}}-\frac{R m N \alpha}{G_{n}}\right]\right)$

where $\alpha$ is given by III-53. 
If the summation in equation IV-10 is approximated as the radial functions $F(r)$ and $d p / d r$ were in section III-4, the expression for the radial electric current simplifies

$$
E_{r}=-\frac{\lambda}{r} e^{-\theta / 2} \frac{2 \pi \sigma \tilde{R b} e^{\theta r^{2} / 2}-\left(1+\frac{\mathrm{FmN}}{\theta}\right)\left(1-e^{-\theta r^{2} / 2}\right) \ln \frac{1}{\varepsilon}-\mathrm{RmN}^{\frac{1-\varepsilon^{2}}{4}} e^{-\theta \frac{1-r^{2}}{2}}}{2 \pi \sigma \tilde{\mathrm{R} b}+\left(1+\mathrm{RmN} \frac{1-e^{-\theta / 2}}{\theta}\right) \ln \frac{1}{\varepsilon}-\mathrm{RmN} \frac{1-\varepsilon^{2}}{4} e^{-\theta / 2}}
$$

The voltage $\Delta \tilde{E}$ applied to the external electric circuit as given by equation II-56 is the negative of the integral of radial electric over the radius from $r=\varepsilon$ to $r=1$ :

$$
\Delta E=\lambda 2 \pi \sigma \mathrm{Rb} \frac{e^{-\theta / 2} \ln \frac{1}{\varepsilon}}{2 \pi \sigma \mathrm{Rb}+\left(1+\mathrm{Rm}^{1} \frac{-e^{-\theta / 2}}{\theta}\right) \ln \frac{1}{\varepsilon}-\mathrm{RmN}^{\frac{1-1}{4}} \mathrm{e}^{-\theta / 2}} \quad \text { IV-12 }
$$

If the external resistance is small compared with the internal resistance, the voltage applied to the external circuit is zero. On the other hand, if the external resistance becomes large compared with the internal resistance, the applied voltage approaches a finite maximum. Equation IV-12 is equivalent to

$$
\tilde{\Delta E}=(2 \pi \sigma \tilde{R} \mathrm{D})(\lambda \alpha)
$$

which is the classic Ohm's law relationship of basic electricity.

The tangential and axial electric flelds are given by equations II-29 and 58 respectively. 
C. MAGNETIC FIELDS

The tangential component of the magnetic field $\mathrm{B}_{\phi}$ may be found from equation II-64. The radial electric current $J_{r}$ is known from equation IV-8. Since the tangential magnetic field is antisymmetric with respect to the plane $z=1 / 2$, it umst satisfy the symmetry condition.

$$
\text { At } z=1 / 2 \quad B_{\phi}=0
$$

Integration of equation IV-8 with respect to $\mathrm{z}$ and application of the above boundary condition yields:

$$
\begin{aligned}
& B_{\phi}=\frac{\lambda a}{r}\left(\frac{1}{2}-z\right) \frac{R m}{s}-\frac{8}{\Pi^{2}} \frac{R m}{s} \frac{\lambda}{r} \sum_{n=1}^{\infty} \frac{\cos (2 n-1) \pi z}{(2 n-1)^{2}}\{\quad I V-14 \\
& \left.\frac{R m N}{G_{n}-\theta}\left[e^{-\theta \frac{1-r^{2}}{2}}-R m N a \frac{1-e^{-\theta \frac{1-r^{2}}{2}}}{\theta}\right]+\left(1-\frac{R m N}{G_{n}-\theta}\right)\left[\left(1+\frac{R m N a}{G_{n}}\right) e^{-G_{n} \frac{1-r^{2}}{2}}-\frac{R m N \alpha}{G_{n}}\right]\right\}
\end{aligned}
$$

where $G_{n}=\theta(2 n-1)^{2}+\operatorname{RmN}$ and $\theta=\pi^{2} s^{2} /$ Re and $\alpha$ is given by equation III-53.

This solution for the tangential magnetic fleld is valid within the region between the two plates. At the boundaries, the electric current flowing through the external circuit causes a jump in the value of $B_{\phi}$ as it crosses the boundaries.

In a manner similar to the solution for the tangential magnetic field, the radial magnetic field $B_{r}$ may be found by a combination of equations II-65, II-67 and III-57. The radial magnetic fleld is also antisymmetric with respect to the mid plane $z=1 / 2$ and must satisfy 
At $z=1 / 2$

$$
B_{r}=0
$$

The resulting solution for the zeroth order radial magnetic field is

$B_{r}=\frac{R m}{s} \frac{8}{\pi^{2}} \sum_{n=1}^{\infty} \frac{\cos (2 n-1) \pi z}{(2 n-1)^{2}}\left\{-\frac{1}{r}+\lambda^{2}\left(1-\frac{F m N}{G_{n} \theta}\right) i\left(1-\frac{R m N \alpha}{2}\right) \frac{e^{-G_{n} \frac{1-r^{2}}{2}}-e^{-\theta \frac{1-r^{2}}{2}}}{r}\right.$
$+R m N \alpha(1-\operatorname{FrnN} \alpha / 2) \frac{1}{r} \frac{1-e^{-\frac{1-r^{2}}{2}}}{\theta}-\frac{R m N \alpha}{G_{n}}\left(1-\frac{R m N \alpha}{2}\right) \frac{1-e^{-G_{n} \frac{1-r^{2}}{2}}}{r}$

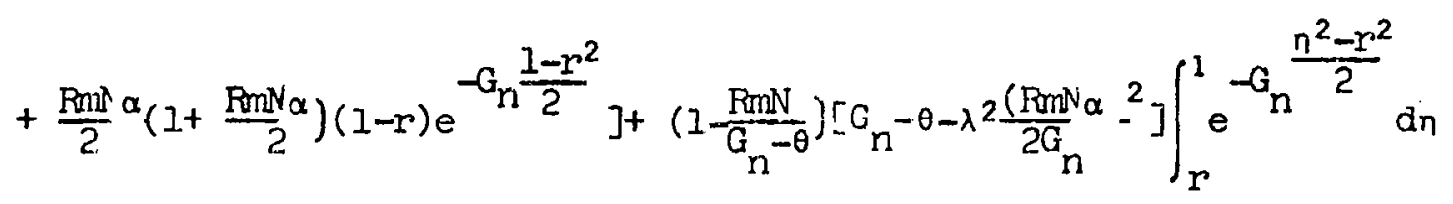
$+\lambda^{2}\left(1-\frac{R m N}{G_{n}-\theta}\right)\left[\left(G_{n}-\theta\right)\left(1-\frac{B m N \alpha}{2}\right)-\frac{R m N \alpha}{2} \cdot\right] \int_{r}^{1}\left[e^{-\theta \frac{1-n^{2}}{2}}-R m N \frac{1-e^{-\theta \frac{1-\eta^{2}}{2}}}{\theta}\right]$ $e^{-G_{n} \frac{n^{2}-r^{2}}{2}} d n t$ IV-15

The change in the radial magnetic fleld $B_{\mathrm{r}}$ from one plate $z=0$ to the other $z=1$ is closely related to the net radial mass flow and $1 \mathrm{~s}$

$$
\Delta B_{r}=B_{r}(r, 1)-B_{r}(r, 0)=\frac{R m}{s r}
$$

The zeroth order axial magnetic field is given by equation II-52, it is just equal to the applied magnetic field. 


\section{DISCUSSION OF RESULTS}

In this chapter, the solutions for the radial and tangential velocities and the temperature, as represented by equations III-57, III-5I and IV-4 respectively, are discussed. These solutions have been calculated numerically on a digital computer and are plotted in figures 2 through 8 for various values of the governing parameters. Additional data concerning the boundary layer thickness, the boundary layer mass flow, the inviscid radial velocity and heat transfer at the walls are given in tables 1 through 4 .

The normalized tangential velocity, $v / \lambda$, is a function of the modified Reynolds number $\operatorname{Re} / \mathrm{s}^{2}$ (or of $\theta$ since $\theta \equiv \pi^{2} \mathrm{~s}^{2} / \mathrm{Re}$ ), the magnetic parameter, $\mathrm{RmN}$, and the external electric current, $a$, as well as the two non-dimensional spatial coordinates $r$ and $z$. The radial velocity, $u$, is a function of all variables just mentioned as well as a function of $\lambda$, the ratio of the inlet tangential velocity to the inlet radial velocity. The temperature is a function of the dimensionless groups $s^{2} / \operatorname{RePr}$ and $\operatorname{RmNEc}\left(1+\lambda^{2} \alpha^{2}\right)$ as well as the coordinates $r$ and $z$.

In all the figures, the velocity components $u$ and $v / \lambda$ and the temperature are shown as functions of the dimensionless axial 
coordinate $z$ for a typical value of the radius, $r=.7$. The shapes of the profiles for other radial values are quite similar to those at $r=.7$ and little would be gatned by considering them in detail. The influence of variations in the radius upon the magnitude of such quantities as boundary layer thickness is shown in the tables. All the figures show the boundary layers which exist on the lower plate; the upper half plane is not shown. The profiles in the upper half plane are 1dentical to those in the lower half plane because of the symmetry of the problem. The value $z=1 / 2$ represents the midplane between the two plates.

\section{The Tangent1al Veloc1ty}

Flgure 2 represents a plot of the normallzed tangential velocity, $v / \lambda$, versus the axial coordinate $z$ for the non-magnetic case, $\mathrm{RmN}=0$. The axial coordinate is plotted vert1cally and the velocity is plotted on the horizontal axis. The profiles are drawn for vartous values of the modifled Reynolds number $\operatorname{Re} / s^{2}\left(6=\pi^{2} s^{2} / \operatorname{Re}\right)$. For large Reynolds numbers ( $\left.\operatorname{small} \theta\right)$, the boundary layers are restricted to very small regions near the plates and the boundary layer blockage is small. This corresponds to case Al discussed in section II-4. Away from the plates, outside the boundary layer, the tangential velocity assumes the potential vortex value of $1 / r$. 
As the Reynolds number is decreased ( $\theta$ increased), the size and influence of the boundary layers grow considerably causing an increase in the boundary layer blockage. Note that for $\theta=1$, the viscous influence extends to the midplane between the two plates, $z=1 / 2$, at $r=.7$. In this case the end wall boundary layers grow rapldily as the radius is decreased from $r=1$; they meet at the midplane between the two plates at some radius greater than $r=.7$. For radial values less than the value where the boundary layers meet, no inviscid core exists. The boundary layers block the flow and the vortex is significantly slowed. This corresponds to case A2 in section II-4. Comparison will be made with the momentum Integral analysis by Loper [8] in the discussion of the table below.

Figure 3 is a plot of the normalized tangentlal velocity, $v / \lambda$, versus the axial coordinate $z$ for values of the magnetic parameters $\mathrm{RmN}$ and a at $\mathrm{r}=.7$ for a typical value of the parameter $\theta: \theta=.01\left(\operatorname{Re} / \mathrm{s}^{2}=100 \pi^{2}\right)$. This figure demonstrates the influence of the magnetic effects upon the tangential velocity. The solid curve marked $\mathrm{RmN}=0$ in figure 3 is the same curve as that marked $\theta=.01$ in flgure 2 and is a typical non-magnetic tangential velocity profile. The remaining profiles show the influence of moderate and large magrietic effects; this corresponds to cases B.1. and $\mathrm{Cl}$ in section $\mathrm{II}-4$.

The curve in figure 3 marked $\mathrm{mmN}=1, \alpha=0$ represents the 
distortion of the tangential velocity distribution with the addition of a moderate magnetic field but with no extemal electric current. The tangential velocity is a bit larger in the boundary layer for this case than it is for the non-magnetic case and the boundary layer is slightly thinner. In the curve marked $\mathrm{RmN}=10$, $\alpha=0$ this filling out of the boundary layer profile is much stronger.

This change in the tangential velocity profile is due to the influence of the electric field which now exists in the fluid. The fluid outside the boundary layers cuts the magnetic field and produce a radial electric field. This electric field is impressed upon the boundary layers exactly as the radial pressure gradient is. Within the slowly moving flufd in the boundary layers, the resulting current combines with the magnetic field creating a Lorentz force. This force acts as a hydromagnetic purmp to speed up the slowly moving boundary layer fluid causing the velocity profile to become fuller. As the velocity profile fills out, the velocity attains its inviscid value at a smaller value of the axial coordinate $\mathrm{z}$ causing a smaller boundary layer thickness. This result is in qualitative agreement with the results of Lewellen and King [6].

If the external resistance of the electric circuit, $\tilde{R}$, is set equal to zero, the current parameter, $\alpha$, attains a maximum limited by the internal resistance of the fluid. The influence of this current upon the tangential velocity profile is shown in the curves 
marked $\mathrm{RmN}=1, \alpha=.74$ and $\mathrm{RmN}=10, \alpha=.222$ in Figure 3. While the application of magnetic fields alone $(\alpha=0)$ does not affect the tangential velocity profile outside the boundary layers, addition of the external current slows the tangential velocity throughout the region of interest. The kinetic energy of the fluid is transformed into electrical energy and is dissipated as joule heating.

For this case of appreciable magnetic fields and currents, the tangential velocity outside the boundary layers assumes its inviscid magnetohydrodynamic value as given by equation A-19. As the inviscid tangential velocity is slowed by the application of the external current, the velocity profile in the boundary layer 1s correspondingly slowed so that the boundary layer thickness remains approximately the same size.

The influence of the independent parameters $r, \mathrm{Re} / \mathrm{s}^{2}, \mathrm{RmN}$ and $\alpha$ upon the boundary layer thickness is shown in Table 1 . The boundary layer thickness $\delta$ is defined as that value of the axial coordinate $\mathrm{z}$ for which the tangential velocity reaches $99 \%$ of 1 ts inviscid value. In the table, the left hand colum gives values of $\theta=\pi^{2} s^{2} /$ Re for three radial stations. The other three colums are values of the boundary layer thickness, $\delta$, for chosen values of the magnetic parameters $\mathrm{RmN}$ and $\alpha$. This table is meant to show the nature of the variation of the boundary layer thickness with these variables and is not intended to cover the entire range of the parameters. 
This table shows that the boundary layers are thinnest near the outer cylinder and thicken as the radius decreases. The growth of the boundary layer thickness with a decrease in Reynolds number (increase in $\theta$ ) still approximates its single plate dependence; that is, the boundary layer thickness is approximately proportional to the inverse root of the Reynolds number. The addition of the magnetic field alone $(\alpha=0)$ decreases the boundary layer thickness by a few percent for the cases tabuiated. The addition of both a magnetic field and external electric current also decreases the boundary layer thickness from its non-magnetic value but not as much as the magnetic field alone does. It appears that for estimation of the boundary layer blockage for the two plate problem, non-magnetic values for the boundary layer thickness may be used as upper bounds for the corresponding magnetic cases over a large parametric range.

In the present analysis, solutions are found only for the zeroth order set of unknowns from equations II-35, which are expansions of the unknowns in inverse powers of the geometric shape parameters.s. Therefore, the solutions obtained are not functions of the parameter, $s$, explicitly. That parameter does appear in the solution equations but always with the Reynolds number as $\mathrm{Re} / \mathrm{s}^{2}$; this may be thought of as a modified Reynolds number not involving s explicitly. Since the solutions obtained above are only the zeroth order solutions and do not depenc explicitly upon the shape parameter, $s$, they cannot be meaningfully compared with the 
results of analyses such as that by Rosenzweig, Lewellen and Ross [9] which do depend explicitly on the parameter $s$. The solutions found in reference 9 are the zeroth order solutions of an expansion in powers of $(1 / s \lambda)^{2}$. This expansion scheme is strictly valid only if

$$
\lambda s>1, \quad s \leq 1 \text { and } \quad \text { Re } \geq 1
$$

(They maintain both $s$ and $\lambda$ as general parameters in their zeroth order solutions by making use of the results of an independent boundary layer analysis by Rott [12]. In patching solutions at the boundary between the inviscid and boundary layer regions, $\lambda$ and $s$ appear independently). At least the first order set of unknowns from the expansions II-35 must be found before meaningful comparisons can be made with analyses which include $s$ as a general parameter.

The present results can be meaningfully compared with the analysis by Loper [8] since that analysis also results in an asymptotic solution without $s$ appearing as an explicit independent parameter. Since that method of solution was a momentum integral analysis, it is most convenient to compare the boundary layer thicknesses predicted by the two analyses. The comparison must be made for the non-magnetic case, $\mathrm{BmN}=0$, since the momentum integral analysis was performed only for this case.

The boundary layer thicknesses for the two cases are compared in Table 2 for a typical value of the radius, $r=.7$. The values of boundary layer thickness for the present analysis (for $r=.7$, 
$\mathrm{RmN}=0$ and $\alpha=0$ ) are listed in the second colum while the first colum lists the corresponding Reynolds number. These results are independent of the inlet velocity ratio $\lambda$ because of the nature of the linearization introduced in chapter III; the radial velocity is uncoupled from the tangential momentum equation. On the other hand, the boundary layer thicknesses found by the momentum integral method do depend upon the inlet velocity ratio $\lambda$. Therefore, agreement between the two methods depends upon the value of $\lambda$ chosen. The results have been compared for two values of $\lambda$, $\lambda=5$ and 10. The case of most interest, a strong vortex, is charactertzed by large values of $\lambda$. The third and fifth columns list the boundary layer thickness as calculated from the momentum integral analysis for $\lambda=5$ and $\lambda=10$ respectively. The fourth and sixth colums list the percent error between the present results and the momentum integral results.

The table shows that the thicknesses are roughly the same order of magnitude but exact agreement is not good; the error is about 50\%. The momentum integral analysis consistentiy predicts thinner boundary layers (for large $\lambda$ ) than the present analysis does. Agreement tends to be better if the inlet velocity ratio $\lambda$ is decreased; this comparison was not pursued further since the case of $\lambda$ small is not of primary interest (and because the calculations are involved and tedious).

It would seem that the present analysis is the more accurate of the two for several reasons. The first is that the accuracy of 
the momentum integral analysis depends upon the proper cholce of velocity profiles. In the two plate momentum integral analysis, plausible profiles are chosen but there is no guarantee that they are accurate representations of the actual profiles. In adaition, in order to obtain a simple closed form solution, it was assumed in the momentum integral analysis that the ratio of the maximum value of the radial velocity within the boundary layers to the inviscid radial velocity outside the boundary layers is a constant. This assumption limited the form of the radial velocity profile; no such assumption was made in the present analysis.

\section{The Radial Velocity}

Figure 4 is the counterpart of figure 2; it plots the radial velocity, $u$, on the horizontal axis versus the axial coordinate $z$ on the vertical axis. This figure is shown for radial station $r=.7$ for the non-magnetic case, $\mathrm{RmN}=0$, for an inlet velocity ration $\lambda=10$. This corresponds to case Al discussed in section II-4. Note that the radial velocity is defined as positive for a radial outflow and therefore is primarily negative in the region of interest. For $r=.7$, the inviscid radial velocity is

$$
\mathrm{u}=-1.429
$$

With the accumulation of radial mass flow within the boundary layers, the radial velocity within the boundary layers becomes quite large, exceeding the inviscid value. This large radial velocity in the boundary layers is referred to as the radial velocity 
overshoot. In order to conserve radial mass flow, the radial flow drawn into the boundary layers must cause a decrease in the outer, inviscid radial velocity.

This effect is shown in figure 4. For large Reynolds number ( $\operatorname{small} \theta$ ) the amount radial mass flow within the boundary layers Is a small fraction of the total mass flow. Thus the outer radial velocity is near its inviscid value of -1.429 . As the Reynolds number is decreased ( $\theta$ increased), the amount of mass flow in the boundary layers increases due to the increasing thickness of the boundary layers. This causes a corresponding decrease in the outer radial velocity. When $\theta=.1$, the boundary layer mass flow is so great as to cause radial back flow outside the boundary layer. This is shown as a positive radial velocity for the curve marked $\theta=.1$ in figure 4. In this case, the vortex motion cannot be maintained and the flow becomes more complicated. The solution equations III-5I, 56, 57 and 58 are no longer strictly valid when this occurs because of the nature of the linearization performed in chapter III. However, it is believed that the analysis may yield meaningful results for very small values of radial backflow (positive radial flow outside the boundary layers). This is based on the fact that for small values of radial backflow, the inviscid tangential velocity is not greatly changed from its value for no backflow. That is, it still is approximately a potential vortex. This is shown in the results of Rosenzweig, Lewellen and Ross [9] (see their figure 7). Note that the maximum value of the radial 
velocity overshoot in the boundary layers is independent of the Reynolds number.

While the maximum value of the radial velocity overshoot is not dependent on the Reynolds number, it is a strong function of the inlet velocity ratio, $\lambda$, which was held constant in figure 4. A value of $\lambda=0$ means that the tangential velocity is zero while $\lambda=10$ means that the inlet tangential velocity is ten times as large as the inlet radial velocity. Figure 5 illustrates this dependence of the radial velocity overshoot upon the velocity ratio $\lambda_{j}$ it is a plot of the radial velocity, $u$, versus the axial coordinate, $z$, at radial station $r=.7$ for a typical value of $\theta, \theta=.01$ $\left(\mathrm{Re} / \mathrm{s}^{2}=100 \pi^{2}\right)$, for the non-magnetic case. When $\lambda=0$, no overshoot occurs, as would be expected. In this case, no centrifugal pressure field is set up to cause an overshoot. As the tangential velocity is increased ( $\lambda$ is increased), the overshoot increases dramatically. As the overshoot becomes larger, the boundary layer mass flow correspondingly grows. Thus the external radial velocity becomes smaller and finally reverses, causing the vortex to break down. This occurs for $\lambda=20$ in figure 5. As the velocity ratio is increased, the radial boundary layer thickness increases slightly since larger velocity gradients occur near the outer edge of the boundary layers.

The influence of the magnetic effects upon the radial velocity is shown in figure 6 . It is a plot of the radial velocity, $u$, versus $z$ at $r=.7$ for $\theta=.01\left(\mathrm{Re} / \mathrm{s}^{2}=100 \pi^{2}\right)$ and $\lambda=10$. This 
corresponds to cases $\mathrm{BI}$ and $\mathrm{Cl}$ discussed qualitatively in section II-4. The curve marked $\mathrm{RmN}=0$ is the same as the curve marked $\theta=.01$ in figure 4. With the addition of a moderate magnetic field but no external electric current ( $\mathrm{RmN}=1, \alpha=0)$, the radial velocity overshoot is decreased a bit. This decrease is explained by the fact that the radial velocity must now do work against the magnetic field producing a dissipative electric current in the tangential direction. If an external electric current is allowed to flow ( $\mathrm{RmN}=1, a=.74$ ), the overshoot is lessened even more. This additional decrease in the overshoot is due to the fact that the inviscid tangential velocity is slowed upon production of this external electric current as was shown in figure 3. This in turn decreases the pressure defect which is the driving force of the radial velocity overshoot. As the amount of overshoot is lessened, the boundary layer mass flow decreases and the Inviscid radial velocity correspondingly increases. As the magnetic influence is increased $(\mathrm{RmN}=10, \alpha=0$ and $\mathrm{mnN}=10, \alpha=.222)$, the overshoot is reduced even more. For the case $\mathrm{FmN}=10, \alpha=.222$, the radial velocity profile begins to approximate 1 ts value for very large magnetic parameter as given in equation A-26 in Appendix III.

Table 3 charts the fraction of the radial mass flow carried within the boundary layers and also the inviscid radial velocity for $\lambda=10$ and for various values of the parameters $r, \theta, \mathrm{mmN}$ and a. If the fraction of the boundary layer mass flow is greater than one, the radial velocity outside the boundary layers reverses 
and becomes positive. The figures given for this case are not reliable since the analysis is not strictly valid for positive core radial velocity.

The fraction of radial mass flow drawn into the boundary layers increases as the radius decreases. This means that the tendency for the inviscid radial velocity to reverse and become positive also increases as the radius decreases. Therefore, the radial backflow (positive radial velocity) will occur first at the inner cylinder $r=\varepsilon$. The tendency for radial backf'low to occur is strengthened by decreasing the Reynolds number (increasIng $\theta$ ) as seen from table 3 . This is explained by the fact that the fraction of radial mass flow carried within the boundary layers increases as the Reynolds number decreases. This occurs because the boundary layers are thicker for smaller Reynolds number while the radial velocity overshoot is independent of Reynolds number. The tendency for radial backflow to occur is also strengthened by increasing the inlet velocity ratio, $\lambda$, as seen from figure 5. The physical reason for this strengthening is the counterpart of the phenomenon described above. While an increase in $\lambda$ does not affect the boundary layer thickness, it does increase the radial velocity overshoot causing additional mass flow to be drawn into the boundary layers.

on the other hand, the presence of magnetic fields and electric currents decreases the tendency for radial backflow to occur. This decrease is caused in part by the decrease in the boundary 
layer thickness with the addition of magnetic effects, but the main influence is the decrease in the radial velocity overshoot with the addition of magnetic effects as seen in figure 6. Thus a non-magnetic analysis will predict vortex breakdown sooner than a magnetic analysis will.

\section{The Temperature}

The solution for the temperature is given by equation IV-4 for the case of prescribed end plates temperature and by equation IV-7 for the case of insulated end plates. In general, the temperature profile is a function of the two spatial coordinates $r$ and $z$ and three dimensionless parameters: $T_{w} / T_{R}$, the ratio of the prescribed end wall temperature to the inlet fluid temperature; $\operatorname{RmNEc}\left(1+\lambda^{2} \alpha^{2}\right)$, the magnetic heating parameter; and $\operatorname{Re} \operatorname{Pr} / \mathrm{s}^{2}$, a modified Peclet number.

Before the temperature profiles are plotted and discussed, it is pertinent to determine what range of the magnetic heating parameter $\operatorname{RmNEc}\left(I+\lambda^{2} \alpha^{2}\right)$ is to be expected. The Eckert number, $E c$, is the ratio of the fluid kinetic energy to the fluid thermal energy. For a problem involving power generation it is reasonable to stipulate that they be the same order of magnitude. The kinetic energy of the inlet fluid is of order $V^{2}\left(1+\lambda^{2}\right)$ while the inlet thermal energy is $c T_{R}$. Thus

$$
\mathrm{V}^{2}\left(1+\lambda^{2}\right)=c \mathrm{~T}_{\mathrm{R}}
$$


Or, Introducing the Eckert number,

$$
E c=\frac{1}{1+\lambda^{2}}
$$

The heating parameter may now be written as

$$
\operatorname{RmN} \frac{1+\lambda^{2} \alpha^{2}}{1+\lambda^{2}}
$$

For power generation, the parameter $\lambda$, being the ratio of the inlet tangential velocity to the inlet radial velocity, will be much larger than one. Thus the heating parameter may be approximated by. $\mathrm{RmNa}^{2}$ for this case. Employing equation III-53, giving the external current, $\alpha$, as a function of $\varepsilon, \theta$ and RmN, it is found that the heating parameter is of order one at its maximum value. Therefore, the heating parameter, $\operatorname{RmNEc}\left(1+\lambda^{2} \alpha^{2}\right)$, will be varied from zero (no magnetic heating effects) to one. For convenience the heating parameter $\operatorname{RmNEc}\left(1+\lambda^{2} \alpha^{2}\right)$ will be denoted by $D$ in the figures and in the following discussion.

Figure 7 is a plot of the temperature versus the axial coordinate $\mathrm{z}$ at radial station $\mathrm{r}=.7$ for temperature ratio $\mathrm{T}_{W} / \mathrm{T}_{\mathrm{R}}=1$ for several values of the magnetic heating parameter $D(=\mathrm{RmNEc}$ $\left.\left(1+\lambda^{2} \alpha^{2}\right)\right)$ and the modifled Peclet number, $\operatorname{RePr} / \mathrm{s}^{2}$. If the magnetic heating is zero and the end wall temperature is the same as the inlet temperature, the entire flow fleld is isothermal: $T=1$. The plot of this temperature coincides with the $\mathrm{z}$ axis in figure 7 . As the magnetic heating is increased from zero, the main body of the flufd increases in temperature and thermal boundary layers 
form near the end plates. This effect is shown in the curves marked $D=1, \frac{\pi^{2} s^{2}}{\operatorname{RePr}}=.1$ and $D=1, \frac{\pi^{2} s^{2}}{\operatorname{RePr}}=.01$ in figure 7 . The parameter $\frac{\pi^{2} s^{2}}{\operatorname{RePr}}$ affects the temperature profile just as the parameter $\pi^{2} s^{2}$, The affects the tangentlal velocity profile. For small $\frac{\pi^{2} \mathrm{~s}^{2}}{\operatorname{RePr}}$ the thermal boundary layer is small, the main body of the fluld is unaffected by the cooling of the end plates and the heat transfer is relatively large. If the Prandtl number is of order one, the thermal and velocity boundary layers will be of the same order of magnitude and the cooling effect will only influence the velocity boundary layer region. If it is desired to cool the velocity boundary layers to prevent undesirable loop currents but not to cool the main body of the fluid, the Prandtl number must be of order one.

Figure 8 plots the temperature versus $z$ at $r=.7$ for temperature ratio $T_{W} / T_{R}=.2$ for several values of heating parameter and modified Peclet number, $\operatorname{RePr} / \mathrm{s}^{2}$. Only the temperature ratio was changed from the conditions given for figure 7 . In the case of end walls cooled below the inlet temperature, thermal boundary layers form even for the case of no heating $\left(D=0, \frac{\pi^{2} s^{2}}{\operatorname{RePr}}=.1\right.$ and $\left.D=0, \frac{\pi^{2} s^{2}}{\operatorname{RePr}}=.01\right)$. The thermal boundary layers thicken slightly but remain of the same order of magnitude as the end wall temperature is lowered. Therefore, the boundary layers may be further cooled by lowering the end wall temperature without significantly affecting the temperature of the main body of fluid. 
Table 4 gives the heat transfer at the end plates, $q$, for various values of the governing parameters, $r, T_{W} / T_{R}, D(=R \mathrm{mNE} c$ $\left.\left(1+\lambda^{2} \alpha^{2}\right)\right)$ and $s^{2} / \operatorname{RePr}$. The heat transfer is defined as positive for heat flowing from the fluid to the end plates. The left hand colum of table 4 gives values of $\mathrm{T}_{\mathrm{w}} / \mathrm{T}_{r}$ for three radial stations. The other six columns are values of the heat transfer, $q$, for chosen values of the parameters $D\left(=\operatorname{RmNEc}\left(1+\lambda^{2} \alpha^{2}\right)\right)$ and $\pi^{2} s^{2} / \operatorname{RePr}$. For the cases of no magnetic heating $(D=0)$ and temperature ratio equal to one, there is no heat transfer at the end walls since the fluid is 1sothermal and no axial temperature gradients exist. The heat transfer is larger for the smaller values of $\pi^{2} \mathrm{~s}^{2} / \operatorname{RePr}$ because larger temperature gradients exist at the walls for the case of thin thermal boundary layers. As the magnetic heating parameter is increased, the heat transfer increases since the temperature difference across the boundary layers increases while the boundary layer thickness remains the same.

The change in heat transfer with change in radial station is not as clear cut as the effects described above. This is caused by the fact that there are two influences upon the heat transfer as the radius changes; these influences act in opposite directions. One influence is the thickening of the thermal boundary layers with decrease with radius analogous to the thickening of the veloctty boundary layers. This tends to decrease the heat transfer since the thermal gradients are smaller in the thicker boundary layers. The other influence is the heating of the fluid as the 
radius decreases. The temperature varies as $D$ In $\frac{1}{r}$ outside the thermal boundary layers. Thus as the radius decreases, the temperature difference across the boundary layers increases, tending to cause a larger heat transfer at the end plates. These opposing influences cause the heat transfer to decrease as the radius decreases when magnetic heating effects are small. On the other hand, these influences cause the heat transfer to increase as the radius decreases when magnetic heating effects are large.

4. Concluding Remarks

The primary purpose of the present analysis was to describe the swirling flow (with a net radial mass flow) of a viscous electrically conducting fluid confined between two finite flat plates in the presence of an applied axial magnetic field.

Of primary concern were the details of the velocity flow field including the blockage of the vortex motion by the buildup of boundary layers on the end plates and the redistribution of radial mass flow into these boundary layers. The main assumptions were that the fluid be incompressible with constant properites and that the flow be laminar, steady and axisymmetric.

The analysis was limited to the case of the confining end plates close together compared with their radius. With this geometric limitation, the unknowns were expanded in powers of the ratio of the separation distance to the radius of the plates. It was then possible to eliminate the electromagnetic unknowns from direct 
consideration in solving for the zeroth order set of unknowns. That is, the Navier-Stokes equations, with magnetohydrodynamic effects included, were expressed entirely in terms of velocity components.

These simplified Navier-Stokes equations were linearized and solved for the zeroth order velocity components and pressure gradient. With the velocities known, the zeroth order temperature and electromagnetic variables were calculated. The zeroth order velocity and temperature profiles were obtained in Fourier series form as functions of a number of governing parameters. These Fourier series were calculated numerically on a digital computer and plotted in the figures for various values of these governing parameters.

The resulting profiles were discussed and, where applicable, compared with previous works. It was concluded that for estimation of the boundary layer blockage a non-magnetic analysis will provide an upper bound on the boundary layer thickness for the corresponding magnetic problem. That is, for a given set of conditions, the boundary layer blockage is a maximum for the non-magnetic case.

The dependence of the radial velocity upon the various parameters was analyzed to determine how these parameters affect the redistribution of radial mass flow. It was found that the radial mass flow in the boundary layers was largest for the nonmagnetic cases. As magnetic effects were added, the boundary layer 
mass flow decreased, lessening the tendency for radial back flow (reversal of radial velocity outside the boundary layers) to occur. In the discussion of the temperature solution, it was found that if the Prandtl number is of order one, the velocity boundary layers may be cooled without affecting the main fluid temperature. This effect is important if it is desired to quench the boundary layers to decrease wasteful loop currents. 
REFERENCES

1. Lengye 1, L.L., "The Analysis of a Vortex Type Magnetohydrodynamic Induction Generator", Case Institute of Technology, Plasma Research Technical Report No. A-10, Cleveland, Oct. 1962

2. Kerrebrock, J. L. and R. V. Maghreblian, "Vortex Containment for the Gaseous rission Rocker" Journal of the Aerospace Sciences (September 1961)

3. Mack, L. M., "The Laminar Boundary Layer on a Disk of Finite Radius in Rotating Flow", California Institute of Technology, Jet Propulsion Laboratory, Technical Report 32- 224, Pasadena, May 1962

4. King, W. S., "Momentum Integral Solutions for the Laminar Boundary Layer on a Finite Disk in a Rotating Flow", Areospace Corporation, Report number ATN-63(9227)-3, 1963

5. Rott, $N$ and W. S. Lewellen, "Boundary Layers in Rotating Flows", Aerospace Corporation, Report Number ATN-64 $(9227)-6,1964$

6. Lewellen, W. S. and W. S. King, "The Boundary Layer of a Conducting Vortex Flow over a Disk with an Axial Magnetic Field, "Proc. of the 8th Midwestern Mech. Conf., Pergamon Press (1965).

7. Vogelpohl, G., "Stromung aus Einer Wirbeiquelle Zwischen Ebenen Wanden", Z. Angew. Math. Mech. Band 24, Nr 45, (1944)

8. Loper, D., "An Analysis of Confined Vortex Flows", Case Inst1tute of Technology, Plasma Research Technical Report No. A-3l, Cleveland, (1964).

9. Rosenzwieg, M. L., W. S. Lewellen, and D A. Ross, "Confined Vortex Flows with Boundary-Layer Interaction." Aerospace corporation, Report, No. AT N-64(9227)-2, 1964.

10. Targ, S. M. "Basic Problems of the theory of Laminar Flows" (Osnovniye ZadachiTeoril Laminamikh Techeni). State Publishing House for Technical Theoretical Literature, Moscow (1951) 
11. Tranter, C. J., "Integral Transforms in Mathematical Physics", Methuen's Monographs (1956).

12. Rott, N., "Turbulent Boundary Layer Development on the End Walls of a Vortex chamber", Aerospace Corporation, Report No. ATN - 62(9202) - 1, (1962).

13. McCune, J. E. and C. Donaldson. "On the Magneto gas dynamics of Compressible Vortices", ARS Preprint 1139-60 (1960). 
APPENDIX I

CONVERGENCE PROOF

The purpose of this appendix is to prove that the solution for the radial function $F(r)$ given by equations III-29 and 30 and the solution for the radial pressure gradient $E d p / d r$ given by equations III-4I and 42 converge and thus are the correct solutions.

The solution for $F(r)$ found in section III-2 is

$F(r)=\left[1+\sum_{m=1}^{\infty} K_{F}^{m}\right]\left[\frac{8}{\pi} 2 \frac{1}{r} \sum_{n=1}^{\infty} \frac{1}{(2 n-1)^{2}} e^{-G_{n} \frac{\left(1-r^{2}\right)}{2}}-\frac{\alpha}{r}\right]$

$K_{F}[x(r)]=\frac{8}{\pi} 2^{R m N} \int_{r}^{1} \frac{n^{2}}{r} x(n) \sum_{n=1}^{\infty} e^{-\frac{n\left(\frac{\left.n^{2}-r^{2}\right)}{2}\right.}{(2 n-1)^{2}}} d n$ III-30

where

$\mathrm{G}_{\mathrm{n}}=\pi^{2} \mathrm{~s}^{2} / \operatorname{Re}(2 \mathrm{n}-1)^{2}+\operatorname{RmN}$

Does this expression yield a finite value for $F(r)$, barring singular values of the parameters such as $r=0$ or $\mathrm{RmN}=\infty$ ?

First, analyze the infinite sums which appear in the above equations; they are

$$
\sum_{n=1}^{\infty} \frac{1}{(2 n-1)^{2}} e^{-G_{n} \frac{\left(1-r^{2}\right)}{2}} \text { and } \sum_{n=1}^{\infty} \frac{1}{(2 n-1) 2} e^{-G_{n} \frac{\left(n^{2}-r^{2}\right)}{2}}
$$

The term $\left(1-r^{2}\right)$ is always greater than or equal to zero since $r=1$ marks the outer limit of the region of interest. The term $\left(n^{2}-r^{2}\right)$ 
Is always greater than or equal to zero also since $n$ is a durmy integration variable with $n=r$ as the lower limit. Since $G_{n}$ is always positive,

$$
e^{-G n \frac{1-r^{2}}{2}} \leq 1 \quad \text { and } \quad e^{-G \frac{n^{2}-r^{2}}{2}} \leq 1 \quad \text { A-1 }
$$

This means that each of the infinite sums being considered is less than the sum

$$
\sum_{n=1}^{\infty} \frac{1}{(2 n-1)^{2}}
$$

which is just equal to $\pi^{2} / 8$ :

$\sum_{n=1}^{\infty} \frac{e^{-G_{n} \frac{1-r^{2}}{2}}}{(2 \pi-1)^{2}} \leq \sum_{n=1}^{\infty} \frac{1}{(2 n-1)^{2}}=\frac{\pi^{2}}{8}$

Thus the infinite sums are finite and bounded.

The entire expression

$$
\left(\frac{8}{\pi^{2}} \frac{1}{r} \sum_{n=1}^{\infty} \frac{1}{(2 n-1)^{2}} e^{-G_{n} \frac{\left(1-r^{2}\right)}{2}}-\frac{\alpha}{r}\right)
$$

which appears in equation III-29 above is finite and bounded (excludIng the case $r=0$ ). A finfte number $M_{1}$ may be chosen such that

$$
\left(\frac{8}{\pi^{2}} \frac{1}{r} \sum_{n=1}^{\infty} \frac{1}{(2 n-1)^{2}} e^{-G_{n} \frac{\left(1-r^{2}\right)}{2}}-\frac{\alpha}{r}\right) \leq M_{1}
$$

for all applicable values of the parameters $r, \alpha, s^{2} /$ Re and $R m$. Similarly, the expression

$$
\left(\frac{8}{\pi^{2}} \operatorname{RmN} \frac{n^{2}}{r} \sum_{n=1}^{\infty} \frac{1}{(2 n-1)^{2}} e^{-G_{n} \frac{\left(n^{2}-r^{2}\right)}{2}}\right)
$$


which appears in equation III-30 is finite and bounded (provided $r \neq 0$ ). It may be bounded by a finite number $M_{2}$ for all applicable values of the parameters $n, r, s^{2} / R e$ and $R m$ :

$$
\left(\frac{8}{\pi^{2}} \operatorname{RmN} \frac{n^{2}}{r} \sum_{n=1}^{\infty} \frac{1}{(2 n-1)^{2}} e^{-G_{n} \frac{\left(n^{2}-r^{2}\right)}{2}}\right) \leq M_{2} \quad A-4
$$

Substitution of these bounding expressions, equations III-29 and 30 yield the inequalities:

$$
F(r) \leq \operatorname{RmN}\left[1+\sum_{m=1}^{\infty} K_{F}^{m}\right] M_{1}
$$

where

$$
K_{F}[x(r)] \leq \int_{r}^{1} M_{2} x(n) d n
$$

The first term of the infinite series appearing in equation $A-5$ is of the form

$$
K_{\mathrm{F}}\left[\mathrm{M}_{1}\right]
$$

where $M_{1}$ is a constant. This may be easily evaluated by use of equation $A-6$ to yield

$$
K_{F}\left[M_{1}\right] \leq M_{1} M_{2}(1-r)
$$

The second term of the infinite series in equation $A-5$ is

$$
\mathrm{K}_{\mathrm{F}}^{2}\left[\mathrm{M}_{1}\right]=\mathrm{K}_{\mathrm{F}}\left[\mathrm{K}_{\mathrm{F}}\left(\mathrm{M}_{1}\right)\right] \leq \mathrm{K}_{\mathrm{F}}\left[\mathrm{M}_{1} \mathrm{M}_{2}(1-\mathrm{r})\right]
$$

or using equation A-6 again,

$$
K_{F}^{2}\left[M_{1}\right] \leq M_{1} M_{2}^{2} \quad \frac{(1-r)^{2}}{2 \cdot 1}
$$


Operating in a similar manner, it is easily seen that the general term of the expansion is

$$
K_{F}^{m}\left[M_{1}\right] \leq M_{1} M_{2}^{m} \frac{(1-r)^{m}}{m !} \quad \text { A-10 }
$$

Now equation $A-5$ for $F(r)$ may be written as

$$
F(r) \leq R m N M_{1} \sum_{m \neq 0}^{\infty} \frac{M_{2}{ }^{m}(1-r)^{m}}{m !}
$$

But the infinite sum in this equation is just the power serles expansion for the exponential function. Thus $F(r)$ is bounded by:

$$
F(r) \leq R m N M_{1} e^{M_{2}(1-r)}
$$

This expression for $F(r)$ converges for all finite values of the parameters $R m N, M_{1}, M_{2}$ and $(1-r)$. Thus the solution for $F(r)$ as given by equations III-29 and III-30 is finite and represents the solution to equation III-28.

Now consider briefly the proof that the solution for $E \mathrm{dp} / \mathrm{dr}$ as represented by equations III- $4 I$ and 42 converges. It is now known that $F(r)$ is finite; therefore its integral appearing in equation III-4I is finite. Thus equations III-4I and 42 are in identical mathematical form to the solution equations for $F(r)$ equations III-29 and 30. The proof that the solution for $E \mathrm{dp} / \mathrm{dr}$ converges follows the above proof identically. 


\section{APPENDIX II}

\section{ANALYSIS. OF ERROR}

The purpose of this appendix is to determine the error introduced in Section III-4 by approximating the function

$$
f(x) \equiv \frac{8}{\pi^{2}} \sum_{n=1}^{\infty} \frac{1}{(2 n-1)^{2}} e^{-(2 n-1)^{2} x},
$$

where $x=\pi^{2} s^{2} / \operatorname{Re} \frac{\left(1-r^{2}\right)}{2}$ or $\pi^{2} s^{2} / \operatorname{Re} \frac{\left(n^{2}-r^{2}\right)}{2}$, by a normalization of 1ts first term

$$
f(x)=e^{-x}
$$

This function $f(x)$ was approximated in section III-4 in order to obtain simpler; closed form, solutions for the velocity profiles. This function appears in the solution equations for $F(r)$, equations III-29 and 30, and for E dp/dr, equations III-4I and 42, and represents the influence of the presence of the boundary layers upon these functions. Thus $f(x)$ as given by equation $A-13$ represents the influence of the boundary layers upon the outer flow. If Reynolds number becomes infinite, there are no boundary layers present. For $\operatorname{Re}=\infty, x=0$ and

$$
f(0)=1
$$


Note that if $f^{\prime}(x)$ were approximated by the constant value $f(x)=1$, it would be tantamount to lgnoring the influence of the boundary layers upon the outer flow entirely.

It is expected that the approximation of this function $f(x)$ by another function will have little effect upon the tangential velocity distribution since the boundary layers have no direct physical effect upon the inviscid tangential velocity. On the other hand, the approximation of $f(x)$ will affect the outer radial flow since the boundary layers have a primary effect upon the inviscid radial velocity through conservation of radial mass flow. This effect will be analyzed below.

Turn now to purely mathematical considerations. It is desired to achieve practical closed form solutions for the radial functions $F(r)$ and $E d p / d r$, starting from equations III-28 and III-39 respectIvely, by approximating the function $f(x)$ as given by equation A-13. This function is difficult to approximate by integer powers of $x$ in the region of $x$ small because $d f / d x$ is singular at $x=0$. The function ( $1-c \sqrt{x}$ ) does approximate $f(x)$ remarkably well for small $x$ but unfortunately does not yield closed form solutions for $F(r)$ and $E d p / d r$.

In theory, closed form solutions are possible for $F(r)$ and $E \mathrm{dp} / \mathrm{dr}$ if $\mathrm{f}(\mathrm{x})$ is approximated by a truncation of its infinite series. However, if the function is approximated by even its first two terms the solutions become quite complex and difficult to 
manipulate. Therefore, for ease of calculation, the function must be approximated by only its first term. Since the infinite series is rapidly convergent, the first term of the series approximates the function quite well.

The full function $f(x)$ as given by equation $A-13$. is plotted versus $x$ in figure 9 along with its approximations by the first term and the first two terms of the series. The approximations are normalized so that $f(0)=1$ is satisfied.

The error introduced by approximating the function $f(x)$ by a constant, its first term and its first two terms is platted versus $x$ in figure 10. The error introduced by approximating $f(x)$ by a constant value, in effect ignoring boundary layer effects, becomes unbounded for large $\mathrm{z}$, corresponding to small values of the Reynolds number. The first term approximation keeps the error bounded; it asymptotically approaches $23 \%$ for large $\mathrm{x}$ (small $\mathrm{Re}$ ). If the second term were added to the approximation, the maximum error would drop to $10 \%$

The actual error introduced into the values of the velocity profiles, and particularly into the tangential velocity, are much less than these figures since this is only the error in the effect of the boundary layers on the outer flow; it is not the error of the entire velocity value.

The fact that the function $f(x)$ is not represented entirely accurately does cause some error in the inviscid radial velocity. 
This error may be corrected by calculation of the actual radial mass flow in the boundary layers and computation of the proper inviscid radial velocity by use of the conservation of radial mass flow condition. This method is used in the numerical calculation of the radial velocity profiles. In this way, the error in the radial velocity is much reduced from the values given in flgure 10. 


\section{APPENDIX III}

SPECIAL CASE SOLUTIONS .

In section III-4, solutions are found for the velocity components $u, v$, and $w$ and the pressure gradient $E d p / d r$ and are given by equations III-51,56, 57 and 58. These solution equations are quite general; no restrictions were placed on the parameters governing the flow. These equations are also quite complex; it is not obvious from these equations what the flow pattern is like for extreme values of the governing parameters. In this appendix, the solution equations will be simplified for several of the limiting values of the governing parameters which were discussed qualatatively in section II-4. This will be done to gain a better Idea of the basic nature of the flow pattern existing in the region of Interest for these special cases. Also, for the case of very large Reynolds number, the simplified solution equations may be easily checked with direct solutions of the governing equations II-75,76 and 77

In several of the special cases considered below, the simplified solutions may not satisfy all the prescribed boundary conditions. This will occur when the terms containing the radial or axial derivatives are negligibly small in the region away from 
the boundaries. In these cases, the simplified solutions represent the general flow pattern away from the boundaries where the conditions are not satisfied. Boundary layers will exist on these boundaries, allowing the boundary conditions to be satisfied.

a. First consider the simplification that the assumption of negIigibly small magnetic effects gives. This corresponds to case A discussed in section II-4. The flow pattern is now one of pure viscous flow with no magnetic effects. Equations III-51, 57, 58 and 56 now are:

$u=\frac{4}{\pi} \sum_{n=1}^{\infty} \frac{\sin (2 n-1) \pi z}{(2 n-1)}\left\{-\frac{1}{r}+\frac{\lambda^{2}}{r}\left(e^{-\theta(2 n-1)^{2} \frac{\left(1-r^{2}\right)}{2}}-e^{-\theta \frac{\left(1-r^{2}\right)}{2}}\right)\right.$ A-14

$\left.+\theta\left[(2 n-1)^{2}-1\right] \int_{r}^{1}\left[1+\lambda^{2} e^{-\theta \frac{1-n^{2}}{2}}\right] e^{-\theta(2 n-1)^{2} \frac{\left(n^{2}-r^{2}\right)}{2}} d n\right\}$

$v=\frac{4}{\pi} \frac{\lambda}{r} \sum_{n=1}^{\infty} \frac{\sin (2 n-1) \pi z}{2 n-1} e^{-\theta(2 n-1)^{2} \frac{\left(1-r^{2}\right)}{2}}$ A-15

$w=\frac{4}{\pi^{2}} \sum_{n=1}^{\infty}[\cos (2 n-1) \pi z] \theta\left\{1-\frac{1}{(2 n-1)^{2}}+\lambda^{2}\left[e^{-\theta \frac{\left(1-r^{2}\right)}{2}}-e^{-\theta(2 n \cdot 1)^{2}} \frac{\left(1-r^{2}\right)}{2}\right]\right.$ $\left.-\left[1-\frac{1}{(2 n-1)_{2}}\right]\left[\frac{1}{r}+\theta(2 n-1)^{2} r\right] \int_{r}^{1}\left[1+\lambda^{2} e^{-\theta \frac{\left(1-n^{2}\right)}{2}}\right] e^{-\theta(2 n-1)^{2}} \frac{\left(n^{2}-r^{2}\right)}{2} d n\right\}$

$E \frac{d p}{d r}=\frac{1}{r 3}+\frac{\theta}{r}+\frac{\lambda^{2}}{r^{3}} e^{-\theta \frac{\left(1-r^{2}\right)}{2}}$

where $\theta=\pi^{2} \mathrm{~s}^{2} / \mathrm{Re}$ 
These are the solutions which would have been obtained if the analysis performed in chapters II and III was done for the case $\mathrm{RmN}=0$. These solutions are valid for all Reynolds number $\left(\theta=\pi^{2} \mathrm{~s}^{2} / \mathrm{Re}\right)$ but are more accurate for the large Reynolds number cases than for the small due to the approximation described in Appendix II. The solutions for the tangential and radial velocities A-14 and 15 are plotted in figures 2,4 and 5 for various values of the parameters $\theta$ and $\lambda$.

b. Consider now the case of infinite Reynolds number $(\theta=0)$ for general magnetic parameter. The fluid is now inviscid and the flow pattern is one of inviscid magnetahydrodynamic flow. This corresponds to an extreme ( since $\mathrm{Re}=\infty$ ) example of cases $\mathrm{Al}, \mathrm{Bl}$ and $\mathrm{Cl}$ discussed in section II-4. In this case, the radial terms in the solution equations III-51, 57 and 58 are no longer functions of the summation index $\mathrm{n}$ and the summation may be written as a constant independent of the axial coordinate $\mathrm{z}$. This means that the velocity profiles are independent of $\mathrm{z}$ as would be expected in the inviscid case. Equations III-5I, 56, 57 and 58 now are

$$
\begin{aligned}
& \mathrm{u}=\frac{1}{\mathrm{r}} \\
& \mathrm{v}=\frac{\lambda}{\mathrm{r}}\left[1-\mathrm{RmN \alpha} \frac{\left(1-\mathrm{r}^{2}\right)}{2}\right] \\
& \mathrm{w}=0 \\
& \mathrm{E} \frac{\mathrm{dp}}{\mathrm{dr}}=\frac{1}{\mathrm{r}^{3}}+\frac{\mathrm{RmN}}{\mathrm{r}}+\frac{\lambda^{2}}{\mathrm{r}^{3}}\left[1-\mathrm{RmN \alpha} \frac{\left(1-\mathrm{r}^{2}\right)}{2}\right]^{2}
\end{aligned}
$$


Note that the ability to satisfy the axial boundary conditions has been lost in this simplification. If the original governing equations II-75, 76 and 77 are solved directly for the case $\operatorname{Re}=\infty$, the results are identical to the above equations. This is a check upon the validity of the analysis performed in chapter III.

A rather surprising result is that equation A-19, for the tangential velocity, is identical to equation (15a) in a paper by McCune and Donaldson [12]; their parameters $(J)$ and $(-\mathrm{Su})$ are identical to $(\alpha)$ and $(\mathrm{RmN})$ respectively. Their equation was derived for a compressible fluid with the radial viscous terms considered while the above result was obtained by considering only the linearized inertia and magnetic terms for an incompressible fluid. This is explained by the fact that the compressibility effect doesn't influence the tangential velocity and by the fact that the solution for the tangential velocity is of the form $\left(c_{1} r+c_{2} / r\right)$ which satisfies the radial viscous terms automatically.

$b, 1$. As a special case of case $b$ described above, consider negligibly small magnetic efrects along with an infinite Reynolds number; that is $\mathrm{RmN}=0$ and $R e=\infty$. This case is purely inviscid and hydrodynamic; the result should be a potential vortex. Equations $\mathrm{A}-18,19,20$ and 21 reduce to

$$
\begin{aligned}
& u=-\frac{I}{r} \\
& v=\frac{\lambda}{r}
\end{aligned}
$$




$$
\begin{aligned}
& w=0 \\
& E \frac{d p}{d r}=\frac{1+\lambda^{2}}{r^{3}}
\end{aligned}
$$

These are the well known potential vortex equations.

$\mathrm{b}, 2$. Consider one more special case of case $\mathrm{b}$ described above; let the magnetic parameter $\mathrm{RmN}$ becomes large. This is equivalent to having a very powerful magnetic field or a very high electrical conductivity. This corresponds to case $\mathrm{Cl}$ discussed in sectopn II-4. Note that when RnN becomes large, the external current $\alpha$ becomes small such that the product $R m N a$ remains finite. In this special case equations A-18, 1920 and 21 are now

$$
\begin{array}{ll}
u=\frac{1}{r} & A-26 \\
v=\lambda / r\left[1-R m N \alpha \frac{\left(1-r^{2}\right)}{2}\right] & A-27 \\
w=0 & A-28 \\
\frac{d p}{d r}=\frac{1}{r} & E=R m N
\end{array}
$$

With the exception of the pressure equation, the above set is identical to those in case b. However, the case under consideration is $\mathrm{RmN}=\infty$. In this spectal case, the equation for the external current $\alpha$, equation III-53, becomes, when multiplied by $\mathrm{RmN}$ :

$$
\mathrm{RmNa}=\frac{\ln \frac{1}{\varepsilon}}{\frac{1}{2} \ln \frac{1}{\varepsilon}-\frac{1-\varepsilon^{2}}{4}}
$$

Now the solution for the tangential velocity, A-27, may be written as 


$$
\mathrm{v}=\frac{\lambda}{\mathrm{r}} \frac{\mathrm{r}^{2} \ln \frac{1}{\varepsilon^{2}}+\varepsilon^{2}-1}{\ln \frac{1}{\varepsilon^{2}}+\varepsilon^{2}-1}
$$

This equation states that the tangential velocity $\mathrm{v}$ is equal to the prescribed value $\lambda$ at the outer cylinder $r=1$, diminishes to zero at radius

$$
r^{2}=\frac{1-\varepsilon^{2}}{\ln \frac{1}{\varepsilon^{2}}}
$$

and is negative for radius smaller than $\sqrt{\left(1-\varepsilon^{2}\right) / \ln \frac{1}{\varepsilon^{2}}}$ In fact, this solution for $\mathrm{v}$ has the property that

$$
\int_{\varepsilon}^{1} \int_{0}^{1} v d z d r=0
$$

Since the external current $\alpha$ is proportional to this double integral, it is very small for large magnetic parameter. To explain why this external current $\alpha$ becomes very small as the magnetic parameter becomes very large, consider the original governing equation. For this special case $(\mathrm{RmN}=\mathrm{Re}=\infty)$ the tangential momentum equation III-ll essentially reduces to

$$
\frac{d}{d r}(v r)=\lambda(\mathrm{RmNa}) r
$$

This equation states that the effect of the current, caused by the tangential velocity cutting the magnetic field, in turn causes the tangential velocity to decrease as the radius decreases. The current produced is very small but it is just enough that the slowing action continues beyond the point where the tangential velocity is stagnated. At radial stations further inward, the current and 
magnetic field act as a hydromagnetic pump, forcing the fluid in the other direction.

c. Consider now the case for which the Reynolds number is small $\left(\mathrm{s}^{2} / \mathrm{Re}\right.$ is large) with the magnetic parameter $\mathrm{RmN}$ unspecified. This corresponds to cases A3, B3 and C3 discussed in section II-4. In this case, the inertia terms are negligibly small compared with the viscous terms; boundary layers exist on the outer radial cylinder. This case will be considered from the simplified governing equations rather than from the simplified solution equations for several reasons. The first reason is that for this case, the solution equations retain the complexity of the radial boundary layers and do not simplify significantly. The second reason is that, while the solution equations are valid for small Reynolds number, the error introduced by approximations is a maximum for this case.

The governing equations II-75, 76 and 77 simplify to

$$
\begin{aligned}
& \frac{1}{r} \frac{\partial}{\partial r}(r u)+\frac{\partial w}{\partial z}=0 \\
& \frac{E}{\operatorname{RmN}} \frac{d p}{d r}=\frac{s^{2}}{\operatorname{ReRmN}} \frac{\partial^{2} u}{\partial z^{2}}-u \\
& 0=\frac{s^{2}}{\operatorname{ReRmN}} \frac{\partial^{2} v}{\partial z^{2}}-\frac{\lambda \alpha}{r}-v+\int_{0}^{1} v d z
\end{aligned}
$$

where the momentum equations have been divided by $\mathrm{RmN}$ for convenience of notation.

The solutions to equations $A-35,36$ and 37 satisfying the 
axial no flow boundary conditions are

$$
\begin{array}{ll}
u=-\frac{1}{r}\left[\frac{\sinh (\mathrm{Ha})-\sinh (\mathrm{Haz})-\sinh (\mathrm{Ha}(\mathrm{l}-\mathrm{z}))}{\sinh (\mathrm{Ha})-\frac{2}{\mathrm{Ha}}(\cosh (\mathrm{Ha})-1)}\right. & A-38 \\
v=0 & \mathrm{~A}-39 \\
w=0 & \mathrm{~A}-40 \\
\frac{\mathrm{dp}}{\mathrm{dr}}=-\frac{\mathrm{RmN}}{\mathrm{E}} \frac{1}{\mathrm{r}} \frac{\sinh (\mathrm{Ha})}{\sinh (\mathrm{Ha})-\frac{2}{\mathrm{Ha}}[\cosh (\mathrm{Ha})-1]} & \mathrm{A}-41
\end{array}
$$

where a modified Hartmann number has been introduced:

$$
\mathrm{Ha}=\frac{\sqrt{\operatorname{ReRmN}}}{\mathrm{s}}
$$

In this case the large viscous forces cause the tangential velocity to stagnate while the radial velocity remains non-zero by conservation of radial mass flow. To satisfy the radial boundary condition at $r=1$ on the tangential, and radial, velocity, boundary layers exist on the outer cylinder. This is a case where boundary layers occur because the Reynolds number is small rather than the normal situation of large Reynolds number.

$c, 1$. As a special case of case $c$, consider now that the magnetic parameter is negligibly small compared with the parameter $\mathrm{s}^{2} / \mathrm{Re}$. This corresponds to ordinary slow flow and is associated with a viscous slowly moving fluid. In this case, the modified Hartmann number becomes zero and the variables $u$ and $d p / d r$ must be determ- 
ined by repeated application of l'Hopital's rule. This yields

$$
\begin{array}{ll}
u=\frac{6}{r} z(z-1) & \\
v & =0 \\
w & =0 \\
\frac{d p}{d r}=\frac{12}{r} & E=\frac{s^{2}}{R e}
\end{array}
$$

These solutions may be found directly from equations A-35, 36 and 37 with the magnetic terms neglected.

If the limit $s^{2} / R e=\infty$ is made in the solution for the pressure gradient III-56, the result is

$$
\frac{d p}{d r}=\frac{\pi^{2}}{r} \quad E=\frac{s^{2}}{R e}
$$

This result, when compared with equation $A-46$, verifies the conclusion of Appendix II that the maximum error in the radial functions $F(r)$ and $d p / d r$ is $23 \%$ for small Reynolds number.

d. This final special case solution is a bit divorced from the above considerations. It is an attempt to calculate the boundary layers which exist on the outer radial cylinders for the small Reynolds number case discussed in case $\mathrm{Cl}$ above. This analysis might shed light on the phenomenon of a boundary layer in a low Reynolds number flow.

Rather than enter a boundary layer analysis and patch flow solutions, it is easier to solve the equations with the radial 
viscous terms included in the entire region of interest. The following analysis is valid for $\operatorname{Re} \ll s$. If $\operatorname{Re}=0$ (s) the inertia term $u \frac{\partial u}{\partial r}$ becomes as large as the viscous terms and must be included also.

The governing equations for this case are

$$
\begin{aligned}
& \frac{1}{r} \frac{\partial}{\partial r}(r u)+\frac{\partial w}{\partial z}=0 \\
& \frac{d p}{d r}=\frac{1}{s^{2}} \frac{\partial}{\partial r}\left[\frac{1}{r} \frac{\partial}{\partial r}(r u)\right]+\frac{\partial^{2} u}{\partial z^{2}} \quad E=\frac{s^{2}}{R e} \\
& 0=\frac{1}{s^{2}} \frac{\partial}{\partial r}\left[\frac{1}{r} \frac{\partial}{\partial r}(r v)\right]+\frac{\partial^{2} v}{\partial z^{2}}
\end{aligned}
$$

These equations are still correct to order $1 / \mathrm{s}$. The newly introduced radial viscous terms are of order $1 / \mathrm{s}^{2}$ compared with the axial viscous terms except within the radial boundary layers.

The boundary conditions to be satisfied are equations II-30-

$$
\begin{array}{llll}
a, b, c, & \text { II-3I, II-32-a,b,c. } & & \\
\text { At } z=0 \text { and } z=1 & u=v=w=0 & \text { II-30-a,b,c } \\
\text { At } z=I / 2 & \frac{\partial u}{\partial z}=\frac{\partial v}{\partial z}=w=0 & \text { II }-31 \\
\text { At } r=I & u=-1 & \text { a } & \\
& v=\lambda & \text { b } & \text { II-32 } \\
w=0 & c &
\end{array}
$$

In addition, conditions must be prescribed on the inner radial cylinder since the equations contain two radial derivatives and require two radial boundary conditions. It is assumed that the in- 
fluence of the boundary condition at $r=1$ are negligible at $r=\varepsilon$ and that the velocities are just those given in case $\mathrm{Cl}$.

$$
\begin{aligned}
& \text { At } r=\varepsilon \quad u=\frac{6}{\varepsilon} \quad z(z-1) \quad \text { a }
\end{aligned}
$$

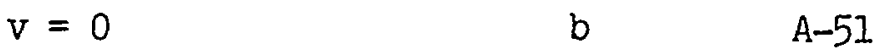

$$
\begin{aligned}
& \begin{array}{ll}
\mathrm{w}=0 & \mathrm{c}
\end{array}
\end{aligned}
$$

Other conditions at the inner cylinder could have been arbitrarily specified; the analysis is not complicated by this condition.

The momentum equations A-49 and 50, are uncoupled and each is linear. Equation A-50 will be solved first since it is homogeneous and points the way for the solution of the non-homogeneous radial momentum equation $A-49$.

Following the pattern of the finite sine transform, assume that the tangential velocity $\mathrm{v}$ is of the form

$$
v=\sum_{n=1}^{\infty} \vec{v}_{n}(r, n) \sin (2 n-1) \pi z
$$

This assumed form for the tangential velocity satisfies the axial boundary conditions automatically.

Substitution of the assumed form A-52 into the tangential momentum equation A-50 yields:

$$
\frac{d^{2} \bar{v}_{n}}{d r^{2}}+\frac{1}{r} \frac{d \vec{v}_{n}}{d r}-\bar{v}_{n}\left(\frac{1}{r^{2}}+(2 n-1)^{2} \pi^{2} s^{2}\right)=0
$$

This equation is easily solved to yield

$$
\bar{v}_{n}=\alpha_{n} I_{1}[(2 n-1) \pi s r]+\gamma_{n} K_{1}[(2 n-1) \pi s r]
$$


where $I_{1}$ and $K_{1}$ are modified Bessel functions.

The constants $\alpha_{n}$ and $\gamma_{n}$ may be determined by use of the radial boundary conditions II-32-b and $A-5 I-b$. This yields

$$
v=\frac{4 \lambda}{\pi} \sum_{n=1}^{\infty} \frac{\sin (2 n-1) \pi z}{2 n-1} \frac{\theta_{n}(r, \varepsilon)}{\theta_{n}(1, \varepsilon)}
$$

where

$\theta_{n}(x, y) \equiv I_{1}[(2 n-1) \pi s x] K_{1}[(2 n-1) \pi s y]-I_{1}[(2 n-1) \pi s y] K_{1}[(2 n-1) \pi s x]$

This is the full solution for the tangential velocity for the case of small Reynolds number and no magnetic field. The profile will be near zero for values of radius away from $r=1$. Near $r=1$ there is a boundary layer like profile.

Now the radial momentum equation A-49 will be solved for the radial velocity in terms of the pressure gradient. Assume a form for $\mathrm{u}$ similar to that assumed for $\mathrm{v}$ in equation $\mathrm{A}-52$; let

$u=\sum_{n=1}^{\infty} \bar{u}_{n}(r, n) \sin (2 n-1) \pi z$ A-57 Again this form satisfies the axial boundary conditions automatically.

Substitution of the assumed form A-57 into the radial momentum equation $\mathrm{A}-49$ yields

$\frac{d^{2} \bar{u}_{n}}{d r^{2}}+\frac{1}{r} \frac{d \bar{u}_{n}}{d r}-\bar{u}_{n}\left(\frac{1}{r^{2}}+(2 n-1)^{2} \pi^{2} s^{2}\right)=\frac{4 s^{2}}{\pi(2 n-1)} \frac{d p}{d r}$ A-58

where use has been made of the fact that 


$$
1=\frac{4}{\pi} \sum_{n=1}^{\infty} \frac{\sin (2 n-1) \pi z}{(2 n-1)}
$$

Equation A-58 has a homogeneous solution identical to equation A-54. The particular solution is easily found by variation of parameters; the complete solution is

$$
\begin{aligned}
& \bar{u}_{n}(r, n)=I_{1}[(2 n-1) \pi s r]\left[v_{n}-\frac{4 s^{2}}{\pi(2 n-1)^{2}} \int_{r}^{1} K_{1}[(2 n-1) \pi s n] n \frac{d p}{d n} d n\right] \\
& +K_{1}[(2 n-1) \pi s r]\left[\omega_{n}-\frac{4 s^{2}}{\pi(2 n-1)^{2}} \int_{\varepsilon}^{r} I_{1}[(2 n-1) \pi s n] n \frac{d p}{d n} d n\right]
\end{aligned}
$$

where the limits of integration have been chosen such that $\bar{u}_{n}$ remains finite as $n \longrightarrow \infty$

The constants of integration $\nu_{n}$ and $\omega_{n}$ are determined by the boundary conditions II-32-a and A-51-a. This yields the solution for the radial velocity:

$u=-\frac{4}{\pi} \sum_{n=1}^{\infty} \frac{\sin (2 n-1) \pi z}{(2 n-1)}\left\{\frac{\theta_{n}(r, \varepsilon)}{\theta_{n}(1, \varepsilon)}+\frac{\theta_{n}(1, r)}{\theta_{n}(1, \varepsilon)} \frac{12}{\varepsilon \pi^{2}(2 n-1)^{2}}\right.$

$\left.+s^{2} \frac{\theta_{n}(1, r)}{\theta_{n}(l, \varepsilon)} \int_{\varepsilon}^{l} \theta_{n}(n, \varepsilon) n \frac{d p}{d n} d n-s^{2} \int_{r}^{l} \theta_{n}(n, r) n \frac{d p}{d n} d n\right\}$

where $\theta_{n}$ is defined by equation $A-56$.

The unknown radial pressure gradient may now be determined by applying the integral form of the conservation of mass equation

$$
\int_{0}^{1} u d z=-\frac{1}{r}
$$


Substitution of the solution for the radial velocity equation $\mathrm{A}-60$ into equation II-80 yields

$$
\begin{aligned}
& \int_{\varepsilon}^{1} n \frac{d p}{d n} \sum_{n=1}^{\infty} \frac{\theta_{n}(1, r) \theta_{n}(n, \varepsilon)}{(2 n-1)^{2} \theta_{n}(1, \varepsilon)} d n-\int_{r}^{1} n \frac{d p}{d n} \sum_{n=1}^{\infty} \frac{\theta_{n}(n, \dot{r})}{(2 n-1)^{2}} d n \\
& +\frac{1}{s^{2}} \sum_{n=1}^{\infty} \frac{\theta_{n}(r, \varepsilon)+\frac{12}{\varepsilon \pi^{2}(2 n-1)^{2}} \theta_{n}(1, r)}{(2 n-1)^{2} \theta_{n}(1, \varepsilon)}-\frac{\pi^{2}}{8 s^{2}} \frac{1}{r}=0 .
\end{aligned}
$$

Equation A-6I is a combination of a Volterra equation of the first kind and a Fredholm equation, also of the first kind. It may be transformed into one of the second kind (with the unknown $\mathrm{dp} / \mathrm{dr}$ appearing explicitly) by differentiating twice with respect to $r$. Once will not suffice since $\theta(r, r)=0$ from the definition. The result is

$$
\begin{aligned}
& \frac{d p}{d r}=8 \sum_{n=1}^{\infty} \frac{\theta_{n}(r, \varepsilon)+\frac{12}{\varepsilon \pi^{2}(2 n-1)^{2}} \theta_{n}(1, r)}{\theta_{n}(1, \varepsilon)}+8 s^{2} \int_{\varepsilon}^{1} \\
& \eta \frac{d p}{d n} \sum_{n=1}^{\infty} \frac{\theta_{n}(1, r) \theta_{n}(n, \varepsilon)}{\theta_{n}(1, \varepsilon)} d n-8 s^{2} \int_{r}^{1} n \frac{d p}{d n} \sum_{n=1}^{\infty} \theta_{n}(n, r) d n
\end{aligned}
$$

Following the method employed in section III-2 to find the solution for the function $F(r)$, the solution for the pressure gradient $d p / d r$ is

$$
\frac{d p}{d r}=8\left[1+\sum_{m=1}^{\infty}\left(K_{3}+K_{2}\right)^{m}\right]\left[\sum_{n=1}^{\infty} \frac{\theta_{n}(r, \varepsilon)+\frac{12}{\varepsilon \pi^{2}(2 n-1)^{2}} \theta_{n}(1, r)}{\theta_{n}(1, \varepsilon)}\right]
$$


where

$K_{3}[x(r)] \equiv s^{2} \int_{\varepsilon}^{1} n x(r) \sum_{n=1}^{\infty} \frac{\theta_{n}(I, r) \theta_{n}(n, \varepsilon)}{\theta_{n}(1, \varepsilon)} d n$

and

$K_{2}[x(r)] \equiv-s^{2} \int_{r}^{1} n x(n) \sum_{n=1}^{\infty} \theta_{n}(n, r) d n$

Now the continuity equation A-48 may be solved for the axial velocity

$$
\begin{aligned}
& w=-\frac{4 s}{\pi} \sum_{n=1}^{\infty} \frac{\cos (2 n-1) \pi z}{(2 n-1)}\left\{\frac{\Psi_{n}(r, \varepsilon)}{\theta_{n}(I, \varepsilon)}+\frac{\Psi_{n}(1, r)}{\theta_{n}(1, \varepsilon)} \frac{12}{\varepsilon \pi^{2}(2 n-1)^{2}}\right. \\
& +s^{2} \frac{\Psi_{n}(1, r)}{\theta_{n}(1, \varepsilon)} \int_{\varepsilon}^{1} \theta_{n}(n, \varepsilon) n \frac{d p}{d n} d n-s^{2} \int_{r}^{1} \Psi_{n}(n, r) n \frac{d p}{d n} d n \\
& \left.+\frac{s^{2}}{(2 n-1) \pi s r} \int_{r}^{1} \theta_{n}(n, r) \frac{d p}{d n} d n\right\}
\end{aligned}
$$

where the variable $\theta_{n}$ is defined by equation $A-56$ and

$$
\begin{array}{r}
\Psi_{n}(x, y) \equiv I_{1}[(2 n-I) \pi s x] K_{0}[(2 n-1) \pi s y]+I_{0}[(2 n-1) \pi s y] K_{1}[(2 n-1) \pi s x] \\
A-67
\end{array}
$$

This solution for the axial velocity satisfies the no flow conditions on the end plates by virtue of the nature of the pressure gradient. However, equation A-66 cannot satisfy the radial boundary conditions II-32-C and A-51-C since the governing differential equations $A-48,49$ and 50 do not contain radial derivations of the axial velocity. 


\section{CALCULATION OF POWER AND EFFICIENCY}

The magneto hydro dynamic device described and analyzed in the main text might be employed as a power generator. Therefore, it is of interest to calculate the performance parameters such as power and efficiency.

\section{Useful Power}

The useful power $\tilde{\mathrm{H}}^{*}$ produced by the device is the product of the external current $\tilde{I}^{*}$ times the voltage $\tilde{\Delta}^{*}$ applied to the extermal circuit. In non-dimensional quantities this may be written as

$$
\tilde{H}=\operatorname{RmN} \tilde{I} \Delta \tilde{E}
$$

The external current is just equal to $2 \pi \lambda \alpha$. The parameter $\alpha$ is given by equation III-53 and the applied voltage $\Delta \tilde{E}$ is given by equation IV-12. Thus the useful power is

$$
\tilde{H}=\frac{\lambda^{2} \mathrm{RmN} 4 \pi^{2} \sigma \tilde{R} \mathrm{~b} e^{-\theta}\left(\ln \frac{I}{\varepsilon}\right)^{2}}{\left[2 \pi \sigma \tilde{R b}+\left(1+R m N \frac{1-e^{-\theta / 2}}{\theta}\right) \ln \frac{1}{\varepsilon}-R m N \frac{1-\varepsilon^{2}}{4} e^{-\theta / 2}\right]^{2}}
$$




\section{Total Power}

The total power expended by the fluid will now be calculated. In the calculation of the temperature distribution, it was assumed that the viscous heating terms were negligible compared with the joule heating terms. This assumption will remain in force for the following calculations.

It is also assumed that the heat conduction effects are negligible. That is, the amount of energy transferred by conduction is assumed to be much smaller than the amount of energy transferred by electromagnetic means. In the case of precribed end wall temperature, heat conduction influences only small thermal boundary layer regions near the two end plates while in the case of adiabatic end plates, there is no heat conduction considered.

With these assumptions in effect, the energy transfer is entirely electromagnetic. The power extracted from the fluid, $H^{*}$, is represented by the dot product of the fluid velocity vector and the magnetic body force vector:

$H^{*}=-\int_{\varepsilon R}^{R} \int_{0}^{b} 2 \pi r^{*} \underline{v}^{*} \cdot\left(\underline{J}^{*} \times \underline{B}^{*}\right) d z^{*} d r^{*}$

where the asterisks indicate dimensional variables and the minus sign is included to make $H^{*}$ positive for power extracted from the fluid. The difference between the useful power and the total power is the ohmic loss of the device which appears as joule heating. In dimensionless form with $\mathrm{H}^{*}=\mathrm{Rb}^{3} \mathrm{~V}^{3} \mathrm{H}$ equation $\mathrm{A}-70$ is 
$H=-2 \pi \operatorname{RnN} \int_{\varepsilon}^{1} \int_{0}^{1} r \underline{v} \cdot(\underline{J} \times \underline{B}) d z d r$

Substituting the expansions of the variables in powers of 1/s from equation II-35 and neglecting terms of order $1 / \mathrm{s}$ or smaller yields

$H=2 \pi R m N \int_{\varepsilon}^{1} \int_{0}^{1} r\left(v_{o} J_{r o}-u_{o} J_{\phi O}\right) d z d r$

By use of equations II -67 and 74 this equation may be written in terms of velocity components only:

$H=2 \pi \operatorname{RmN} \int_{\varepsilon}^{1} \int_{0}^{1} r\left[u_{0}^{2}+v_{0}^{2}-v_{0} \int_{0}^{1} v_{0} d z+v_{0} \frac{\lambda \alpha}{r}\right] d z d r$

Th1s expression for the total power may be found in some generality by use of equations III-5I and 57. However, the result is very cumbersome and difficult to analyze further. In keeping with the previous assumptions concerning the size and influence of the boundary layers, $\mathrm{H}$ may be approximated to the same degree of accuracy that $\tilde{H}, E_{r o}, R(r)$ and $\frac{d p}{d r}$ were:

$H=2 \pi R m N\left\{\ln \frac{1}{\varepsilon}+\frac{\lambda^{2} e^{-\theta}\left(\ln \frac{1}{\varepsilon}\right)^{2}\left(2 \pi \sigma \tilde{R}^{\circ}+\ln \frac{1}{\varepsilon}\right)}{\left[2 \pi \sigma R D+\left(\operatorname{RmN} \frac{1-e^{-\frac{\theta}{2}}}{\theta} \ln \frac{1}{\varepsilon}-R m N \frac{1-\varepsilon^{2}}{4} e^{-\theta / 2]^{2}}\right.\right.}\right\}$ A-74

The first term on the right hand side represents the power lost by the azimuthal current produced by the radial velocity. The other term represents power produced both within the fluld and 
and in the external circuit by the net radial current.

\section{Efficiency}

The efficiency of the device, $B$, may now be expressed as the useful power $\tilde{H}$ divided by the total power $H$ :

$$
\beta=\tilde{\mathrm{H}} / \mathrm{H}
$$

or, using equations $A-69$ and $A-74$;

$B=\frac{\lambda^{2} 2 \pi \sigma \tilde{R D} e^{-\theta} \ln \frac{1}{\varepsilon}}{\left[\left(2 \pi \sigma \tilde{R D}+\left(1+R m N \frac{1-e^{-\theta / 2}}{\theta}\right) \ln \frac{1}{\varepsilon}-R m N \frac{1-\varepsilon^{2}}{4} e^{-\theta / 2}\right)^{2}+\lambda^{2} e^{-\theta} \ln \frac{1}{\varepsilon}\left(2 \pi \sigma \tilde{R b}+\ln \frac{1}{\varepsilon}\right)\right]}$ A-76 


\section{Table 1 Boundary Layer Thickness}

The boundary layer thickness, $\delta$, is defined as the value of the axial coordinate $\mathrm{z}$ for which the tangential velocity, $\mathrm{v}$, is equal to $99 \%$ of its inviscid value.

\begin{tabular}{|c|c|c|c|c|}
\hline & $\theta=\frac{\pi^{2} 2}{\operatorname{Re}}$ & $\begin{aligned} \mathrm{RmN} & =0 \\
\alpha & =0\end{aligned}$ & $\begin{aligned} \mathrm{RmN} & =1 \\
\alpha & =1\end{aligned}$ & $\begin{aligned} \operatorname{RonN} & =1 \\
\alpha & =.74\end{aligned}$ \\
\hline$r=.9$ & $\begin{array}{l}.0001 \\
.001 \\
.01 \\
.1\end{array}$ & $\begin{array}{l}.00497 \\
.01288 \\
.0361 \\
.1288\end{array}$ & $\begin{array}{l}.00494 \\
.01238 \\
.03487 \\
.1239\end{array}$ & $\begin{array}{l}.004945 \\
.01269 \\
.03557 \\
.1271\end{array}$ \\
\hline$r=.7$ & $\begin{array}{l}.0001 \\
.001 \\
.01 \\
.1\end{array}$ & $\begin{array}{l}.0082 \\
.01928 \\
.0588 \\
.1928\end{array}$ & $\begin{array}{l}.0078 \\
.01894 \\
.0573 \\
.1893\end{array}$ & $\begin{array}{l}.00816 \\
.01922 \\
.0586 \\
.1922\end{array}$ \\
\hline$r=.2$ & $\begin{array}{l}.0001 \\
.001 \\
.01 \\
.1\end{array}$ & $\begin{array}{l}.0096 \\
.0257 \\
.0804 \\
.257\end{array}$ & $\begin{array}{l}.00942 \\
.02446 \\
.0763 \\
.2447\end{array}$ & $\begin{array}{l}.00959 \\
.02558 \\
.0801 \\
.2543\end{array}$ \\
\hline
\end{tabular}


Table 2 Comparison of Boundary Layer Thicknesses

The boundary layer thickness for the non-magnetic case as given in Table 1 is compared at $r=.7$ with the momentum integral solution [8] for various values of modffied Reynolds number and inlet velocity ratio

$$
\mathrm{RmN}=0 \quad \mathrm{r}=.7
$$

\begin{tabular}{l|l|l|l|l|l}
$\frac{\pi^{2} \mathrm{~s}^{2}}{\mathrm{Re}}$ & $\begin{array}{l}\text { BLT. from } \\
\text { present analysis }\end{array}$ & $\begin{array}{l}\text { M. I. BLT } \\
\text { ror } \lambda=5\end{array}$ & $\begin{array}{c}\% \\
\text { error }\end{array}$ & $\begin{array}{l}\text { M. I. BLT } \\
\text { for } \lambda=10\end{array}$ & $\begin{array}{c}\% \\
\text { error }\end{array}$ \\
\hline .0001 & .0082 & .00336 & 59.2 & .00245 & 70.2 \\
.001 & .01928 & .01055 & 45.3 & .00775 & 59.3 \\
.01 & .0588 & .0327 & 44.4 & .025 & 57.5 \\
.1 & .1928 & .1111 & 42.4 & .0828 & 57.2
\end{tabular}

BLT $=$ Boundary Layer thickness

M.I. = Momentum integraI 
Table 3 Boundary Layer Mass Flow and Inviscid Radial Velocity

The boundary layer mass flow is defined as the fraction of the total input radial mass flow which is carried within the boundary layers. If this fraction becomes greater than one, the inviscid radial velocity will become positive. In this case the vortex breaks down and the flow pattern becomes more complex. If this occurs, the analysis is not valid and the data is not rellable. This table is computed for an inlet velocity ratio of ten.

\begin{tabular}{|c|c|c|c|c|c|c|c|}
\hline & \multirow{2}{*}{$\theta=\frac{\pi^{2} s^{2}}{R e}$} & \multicolumn{2}{|c|}{$\mathrm{RmN}=0, \alpha=0$} & \multicolumn{2}{|c|}{$\mathrm{RmN}=1, a=0$} & \multicolumn{2}{|c|}{$\mathrm{RmN}=1, \alpha=.74$} \\
\hline & & $\mathrm{BLMF}$ & $u_{\text {Inv }}$ & BLMF & $u_{\text {inv }}$ & BLMF & $u_{\text {inv }}$ \\
\hline$r=.9$ & $\begin{array}{l}.0001 \\
.001 \\
.01 \\
.1\end{array}$ & $\begin{array}{l}.0143 \\
.0434 \\
.1287 \\
.429\end{array}$ & $\begin{array}{l}-1.1062 \\
-1.0907 \\
-1.044 \\
-0.854\end{array}$ & $\begin{array}{l}.01388 \\
.0420 \\
.1239 \\
.415\end{array}$ & $\begin{array}{l}-1.1066 \\
-1.092 \\
-1.048 \\
-0.8713\end{array}$ & $\begin{array}{l}.01343 \\
.0396 \\
.7167 \\
.388\end{array}$ & $\begin{array}{l}-1.1071 \\
-1.094 \\
-1.055 \\
-0.904\end{array}$ \\
\hline & $\left(\begin{array}{l}.0001 \\
.001 \\
.01 \\
.1\end{array}\right.$ & $\begin{array}{l}.0555 \\
.1607 \\
.5230 \\
.54\end{array}$ & $\begin{array}{l}-1.37 \\
-1.24 \\
-0.774 \\
+1.26\end{array}$ & $\begin{array}{l}.0494 \\
.149 \\
.464 \\
1.38\end{array}$ & $\begin{array}{l}-1.38 \\
-1.26 \\
-0.865 \\
+0.87\end{array}$ & $\begin{array}{l}.0421 \\
.1236 \\
.384 \\
1.104\end{array}$ & $\begin{array}{l}-1.39 \\
-1.30 \\
-1.00 \\
+0.33\end{array}$ \\
\hline & $\left\{\begin{array}{l}.0001 \\
.001 \\
.01 \\
.1\end{array}\right.$ & $\begin{array}{l}.269 \\
.878 \\
2.85 \\
7.35\end{array}$ & $\begin{array}{r}-3.76 \\
-0.64 \\
+10.4 \\
+65.0\end{array}$ & $\begin{array}{l}.218 \\
.718 \\
2.25 \\
6.13\end{array}$ & $\begin{array}{l}-3.98 \\
-1.48 \\
+7.4 \\
+50.0\end{array}$ & $\begin{array}{l}.126 \\
.407 \\
1.27 \\
3.50\end{array}$ & $\begin{array}{c}-4.45 \\
-3.13 \\
+1.6 \\
+25.0\end{array}$ \\
\hline
\end{tabular}

BLMF = Boundary layer mass flow $u_{\text {Inv }}=$ inviscid radial velocity 
Table 4 Heat Transfer at the End Plates

The heat transfer at the end plates, $q$, is defined as positive for heat flowing from the fluid to the end plates. If the end plates are insulated, the heat transfer is zero.

$\operatorname{RmNEc}\left(1+\lambda^{2} \alpha^{2}\right) \equiv D$

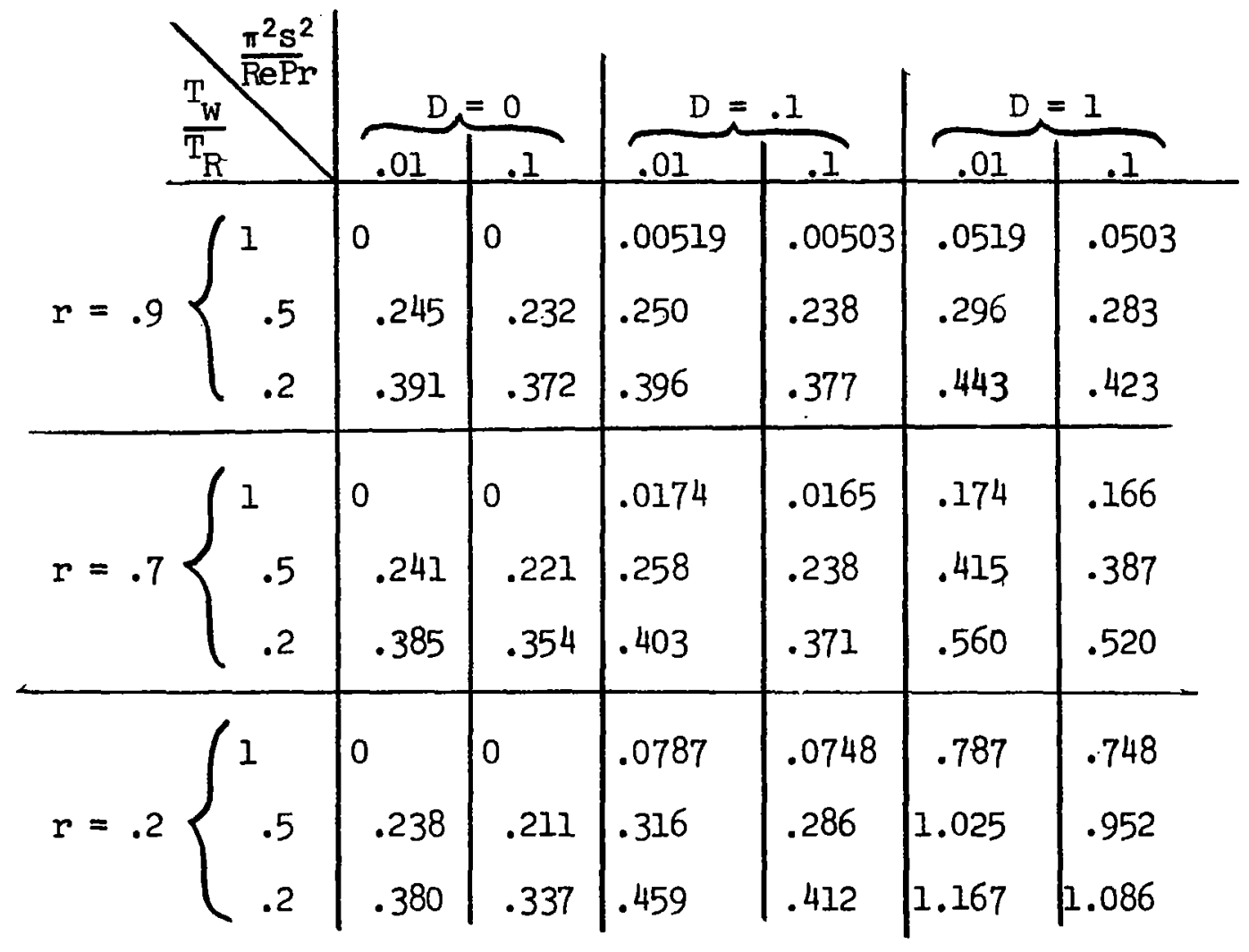




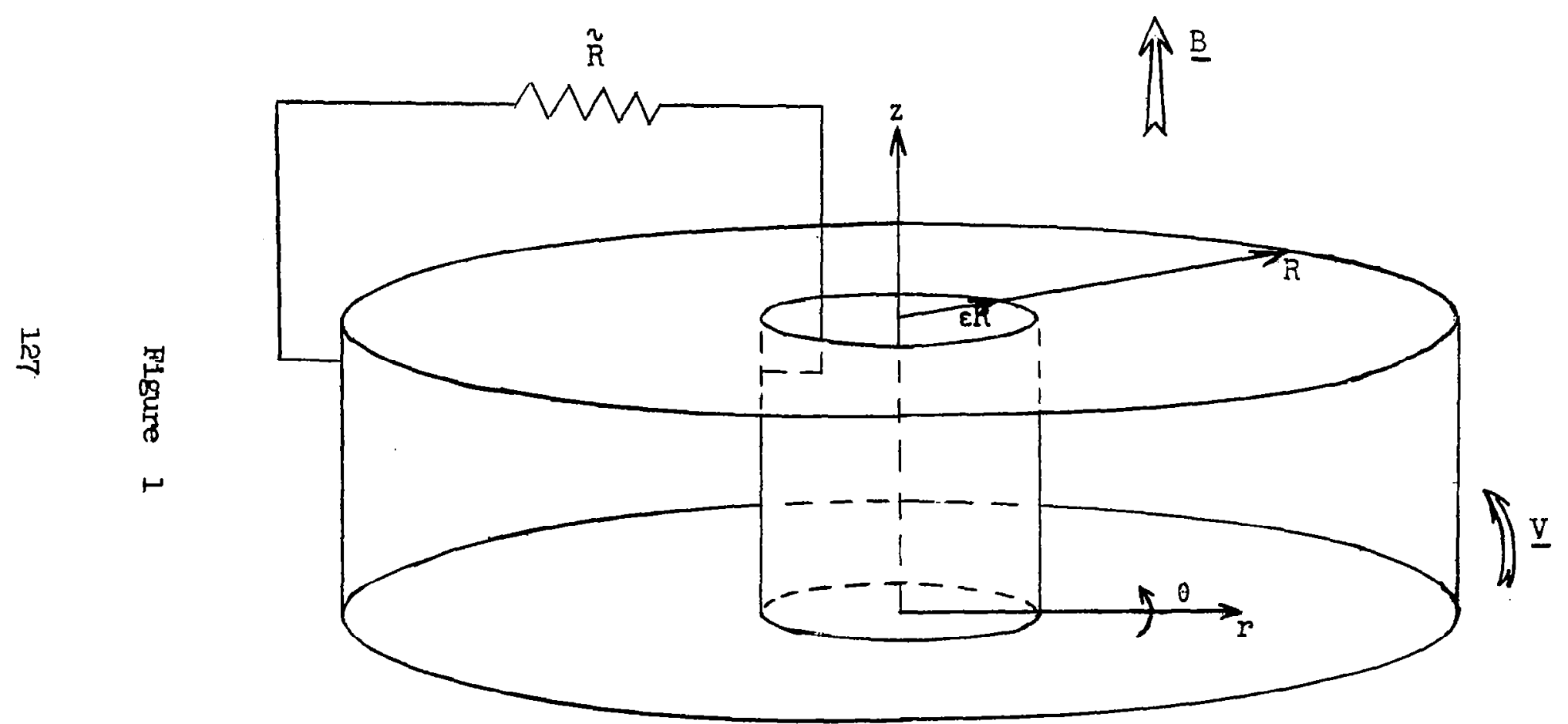




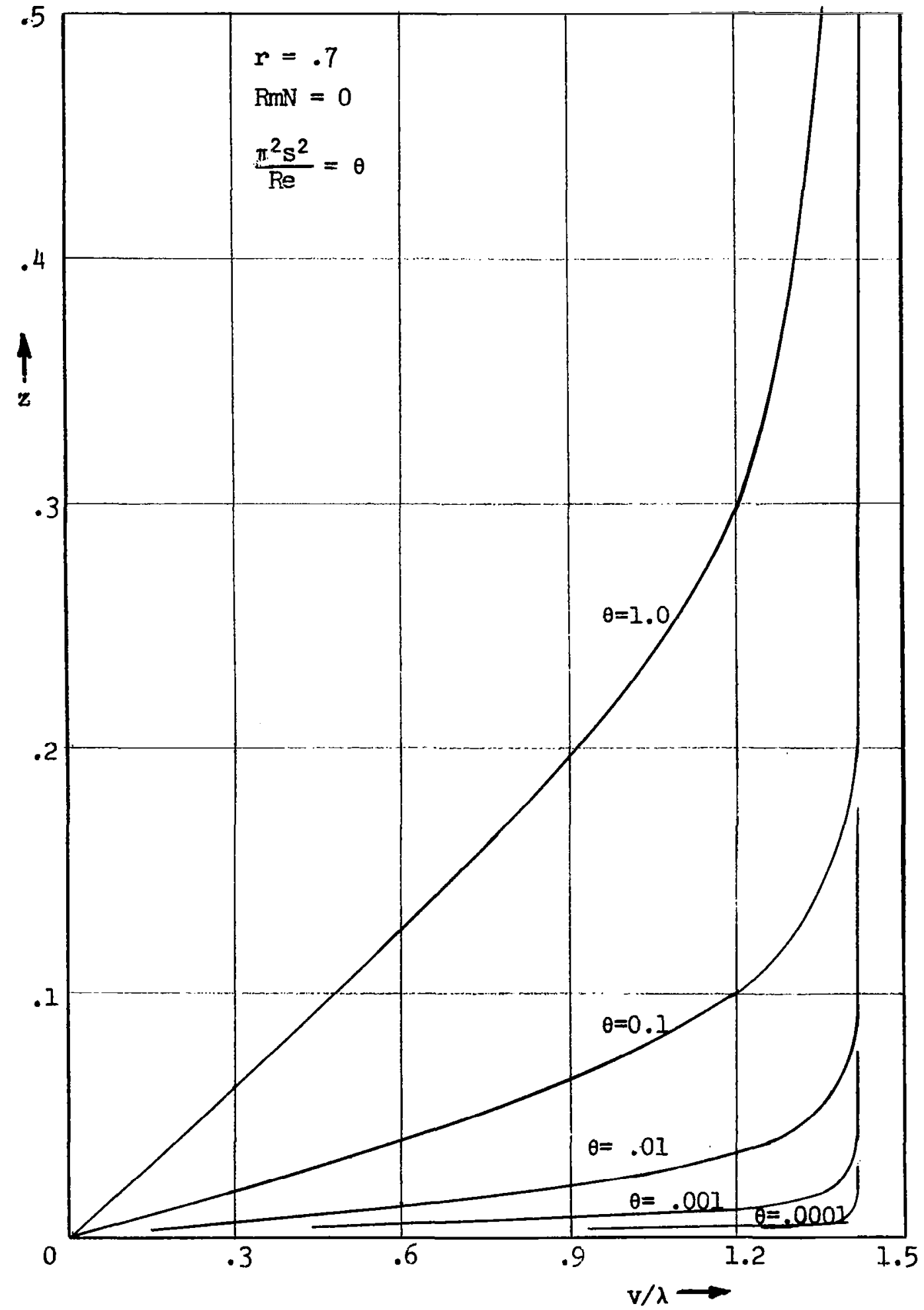

Figure 2 


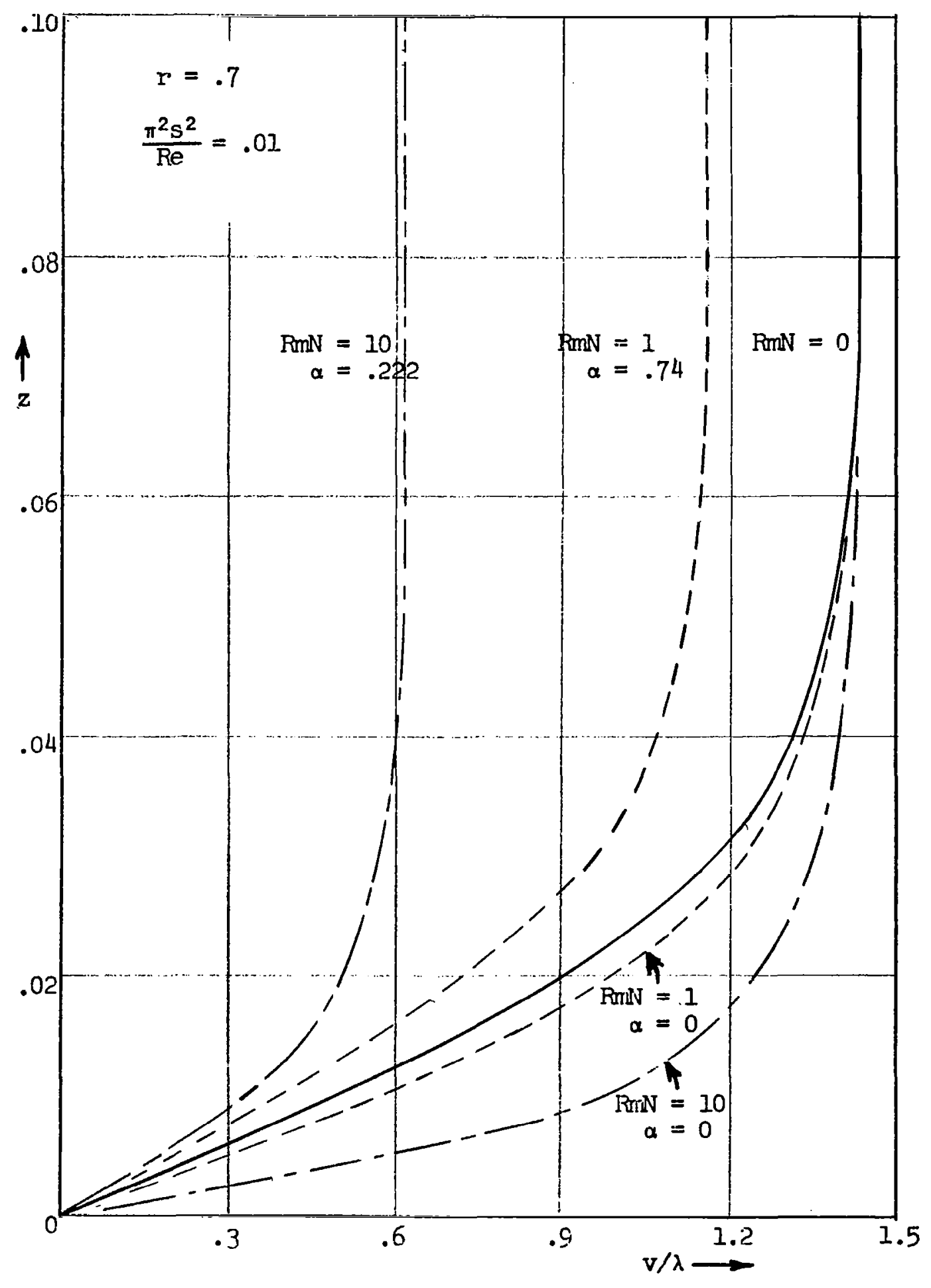

Figure 3 


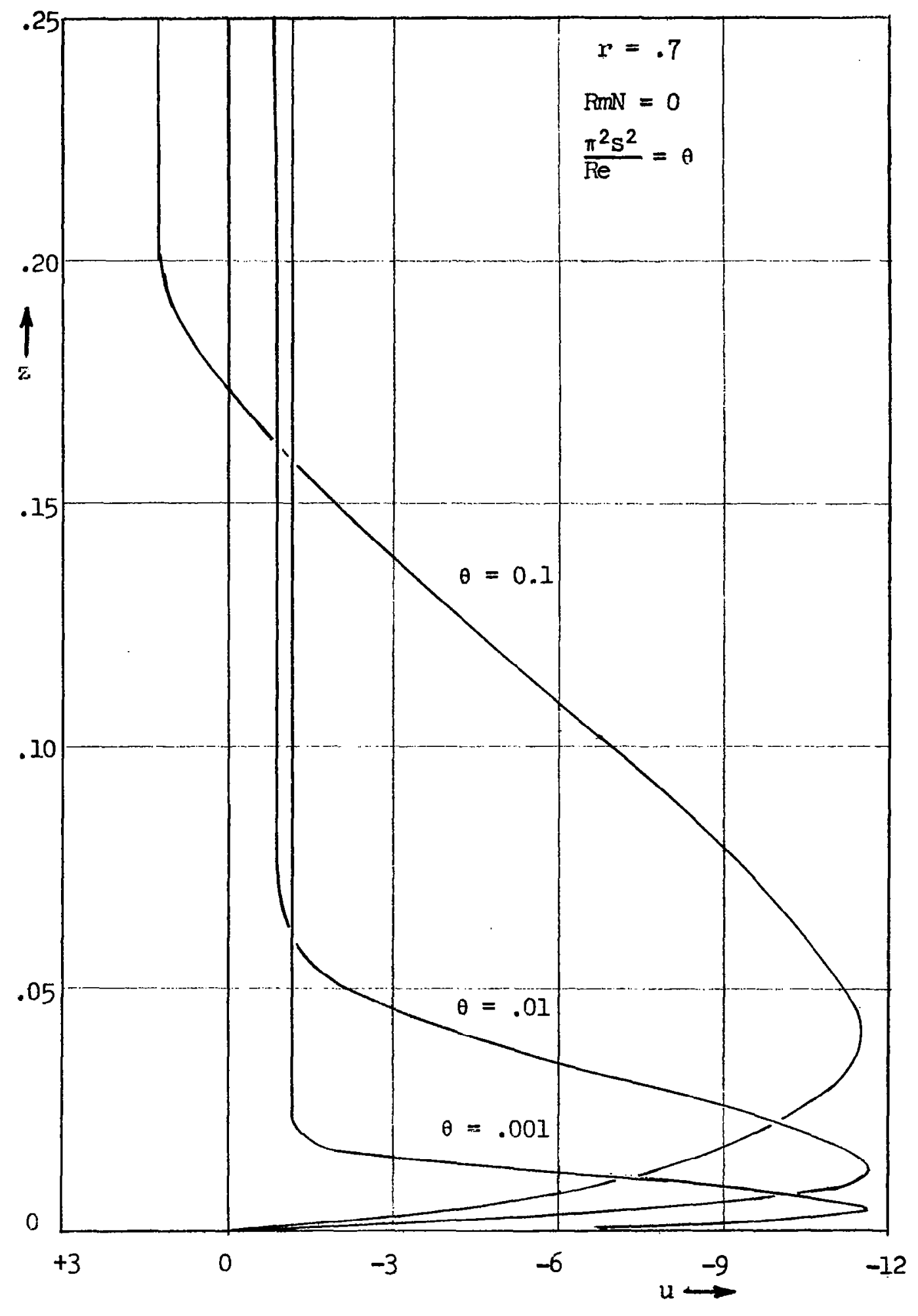

Figure 4 


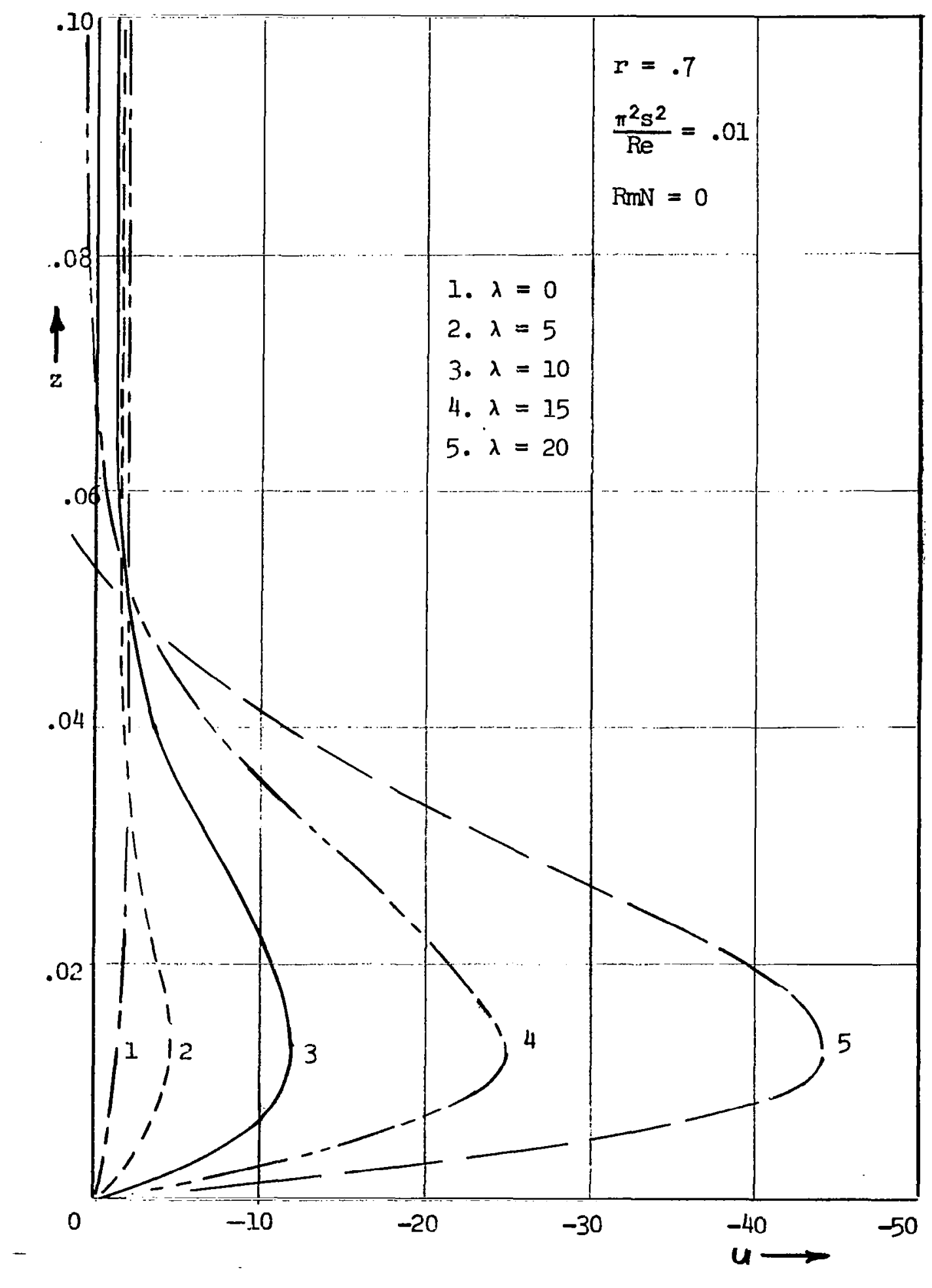

Flgure 5 


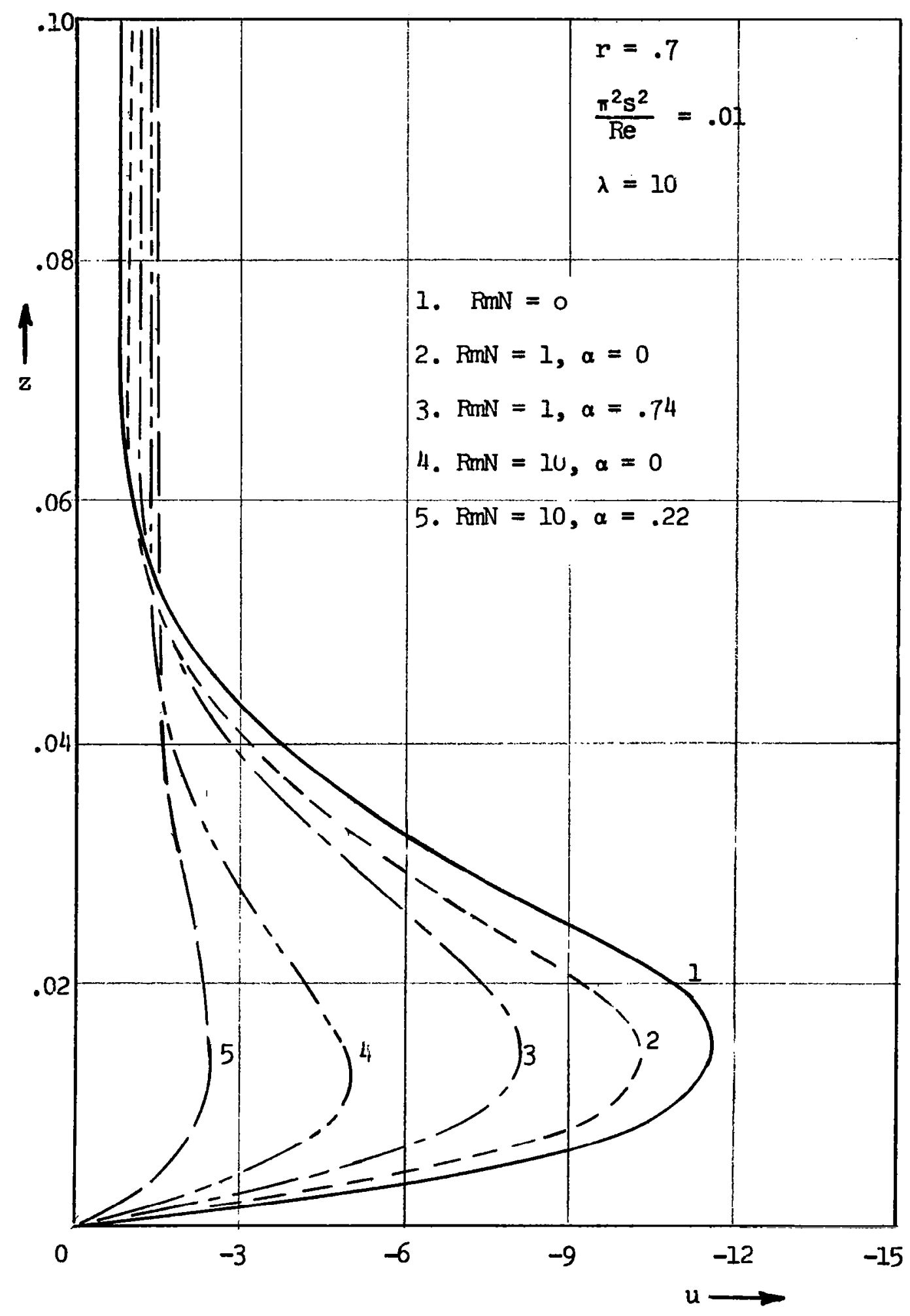

Figure 6 


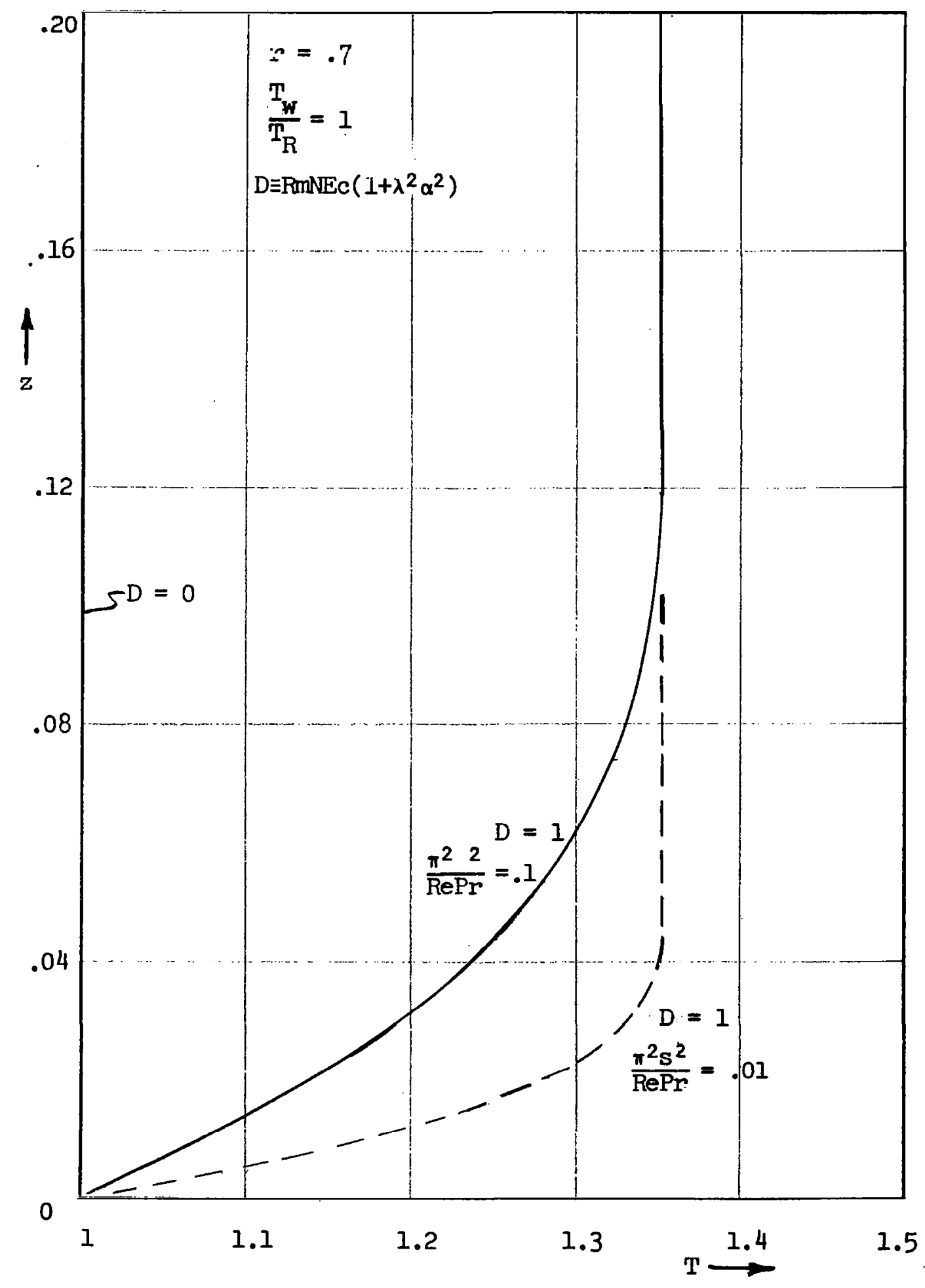

Figure 7 


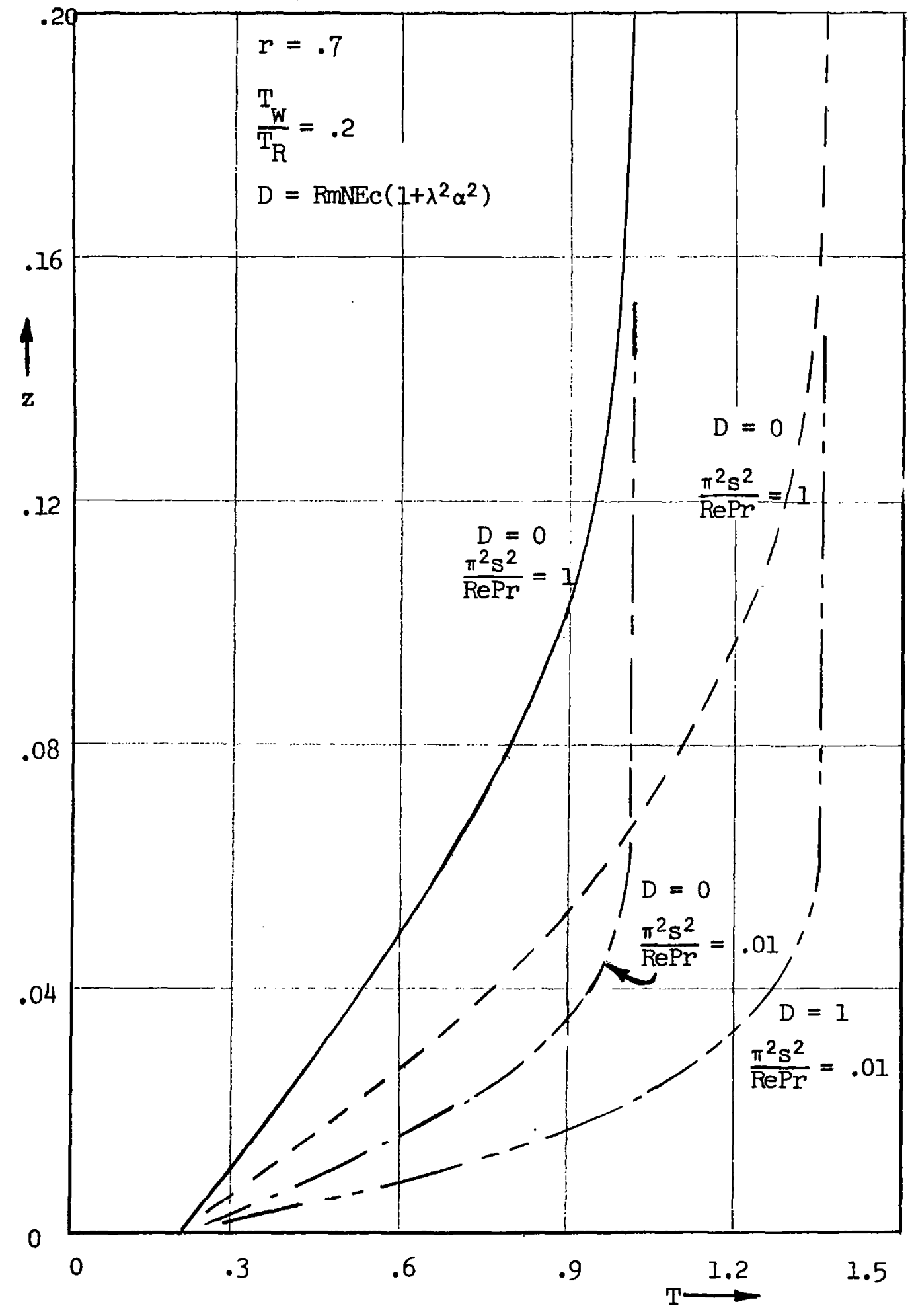

Figure 8 


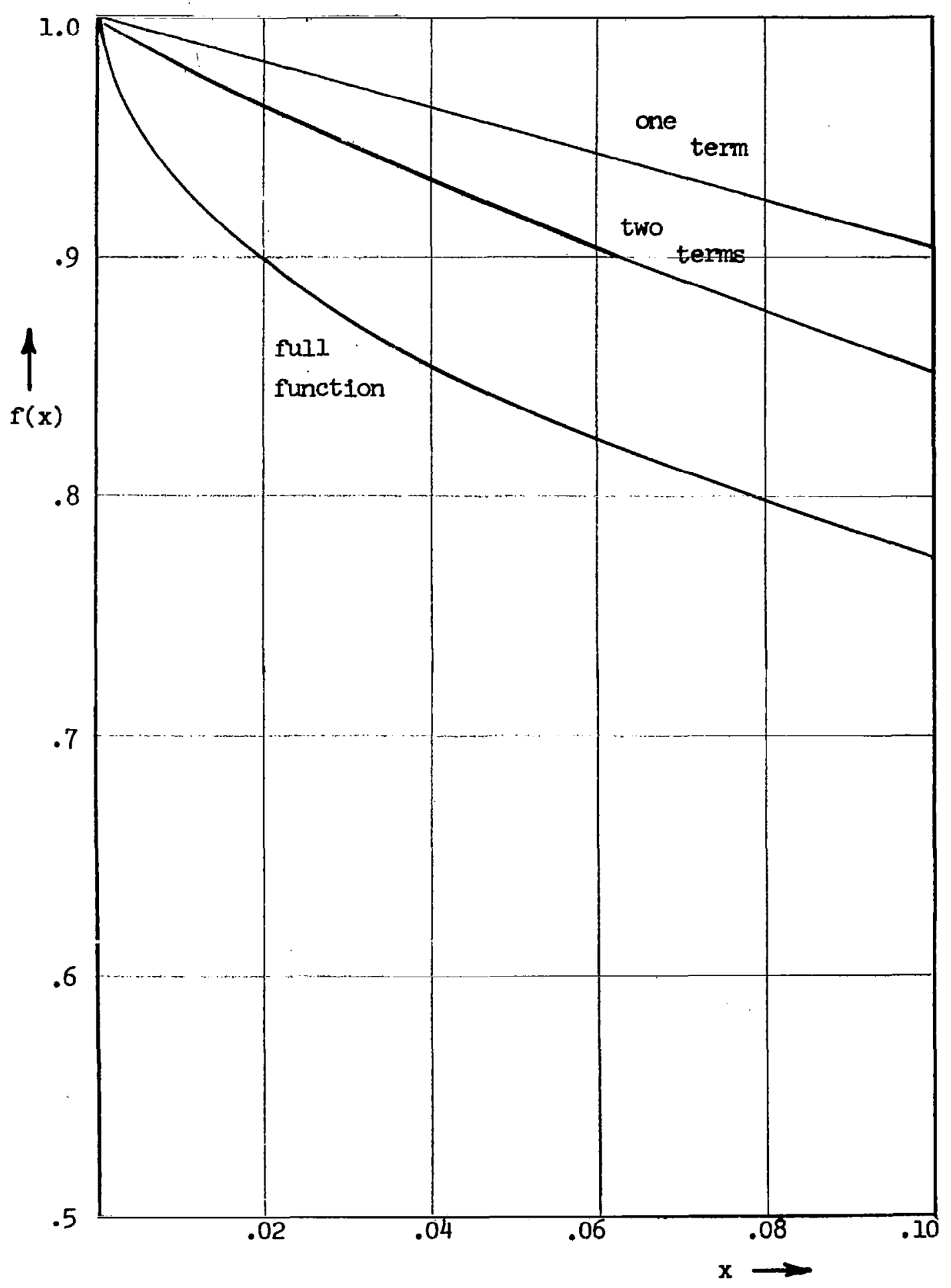

Figure 9 


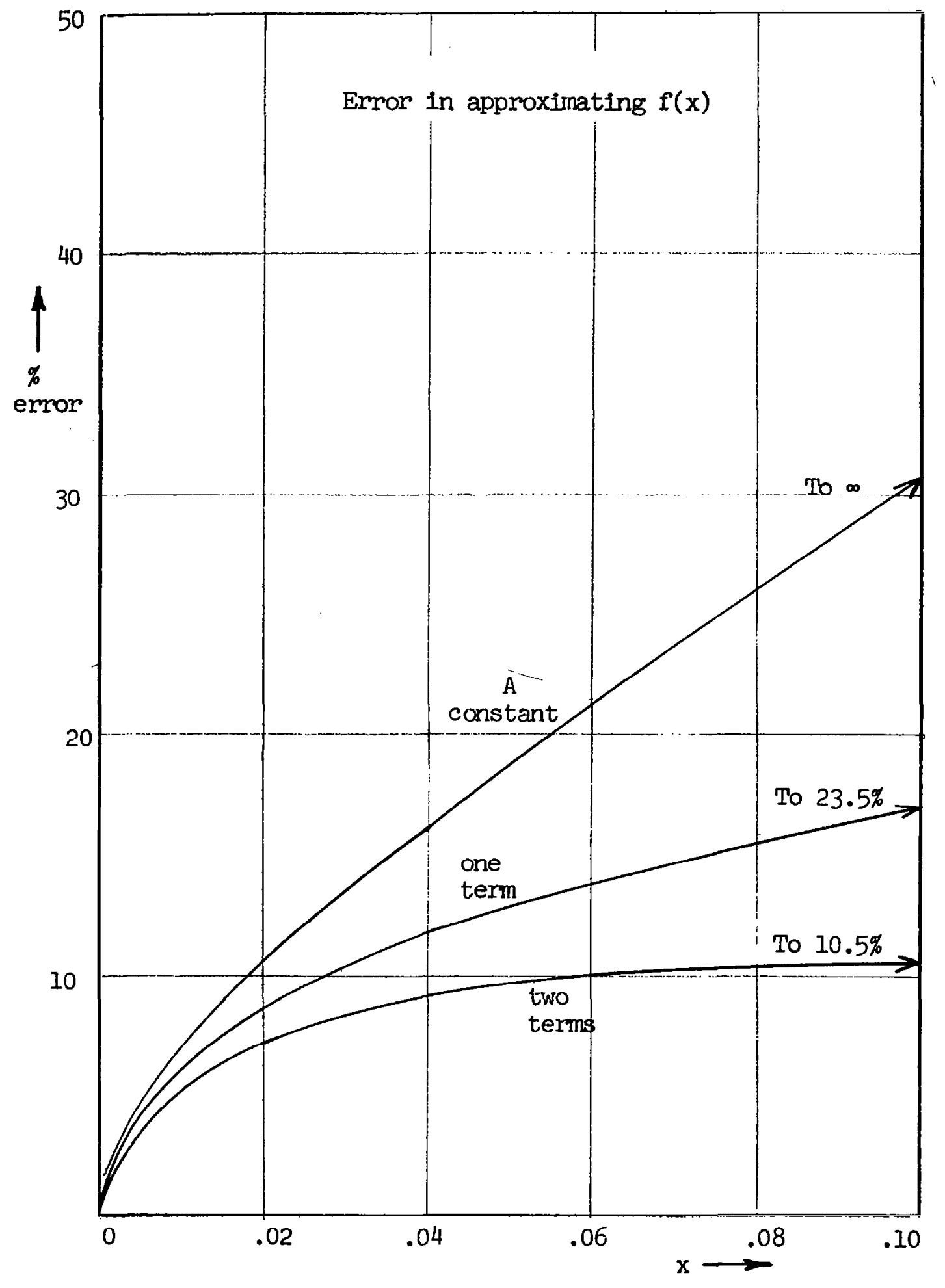

Figure 10 